\title{
QUANTUM CAUSALITY, DECOHERENCE, TRAJECTORIES AND INFORMATION
}

\author{
V P BELAVKIN
}

In celebration of the 100th anniversary of the discovery of quanta

\begin{abstract}
A history of the discovery of "new" quantum mechanics and the paradoxes of its probabilistic interpretation are briefly reviewed from the modern point of view of quantum probability and information. The modern quantum theory, which has been developed during the last 20 years for treatment of quantum open systems including quantum noise, decoherence, quantum diffusions and spontaneous jumps occurring under continuous in time observation, is not yet a part of the standard curriculum of quantum physics. It is argued that the conventional formalism of quantum mechanics is insufficient for the description of quantum events, such as spontaneous decays say, and the new experimental phenomena related to individual quantum measurements, but they all have received an adequate mathematical treatment in quantum stochastics of open systems.
\end{abstract}

Moreover, the only reasonable probabilistic interpretation of quantum mechanics put forward by Max Born was in fact in irreconcilable contradiction with traditional mechanical reality and causality. This led to numerous quantum paradoxes, some of them due to the great inventors of quantum theory such as Einstein and Schrödinger. They are reconsidered in this paper from the point of view of quantum information.

The development of quantum measurement theory, initiated by von Neumann, indicated a possibility for resolution of this interpretational crisis by divorcing the algebra of the dynamical generators and the algebra of the actual observables, or beables. It is shown that within this approach quantum causality can be rehabilitated in the form of a superselection rule for compatibility of the past beables with the potential future. This rule, together with the self-compatibility of the measurements insuring the consistency of the histories, is called the nondemolition, or causality principle in modern quantum theory. The application of this rule in the form of the dynamical commutation relations leads to the derivation of the von Neumann projection postulate, and also to the more general reductions, instantaneous, spontaneous, and even continuous in time. This gives a quantum stochastic solution, in the form of the dynamical filtering equations, of the notorious measurement problem which was tackled unsuccessfully by many famous physicists starting with Schrödinger and Bohr.

It has been recently proved that the quantum stochastic model for the continuous in time measurements is equivalent to a Dirac type boundary-value problem for the secondary quantized input "offer waves from future" in one extra dimension, and to a reduction of the algebra of the consistent histories of past events to an Abelian subalgebra for the "trajectories of the output particles". This supports the corpuscular-wave duality in the form of the thesis that everything in the future are quantized waves, everything in the past are trajectories of the recorded particles. 


\section{Contents}

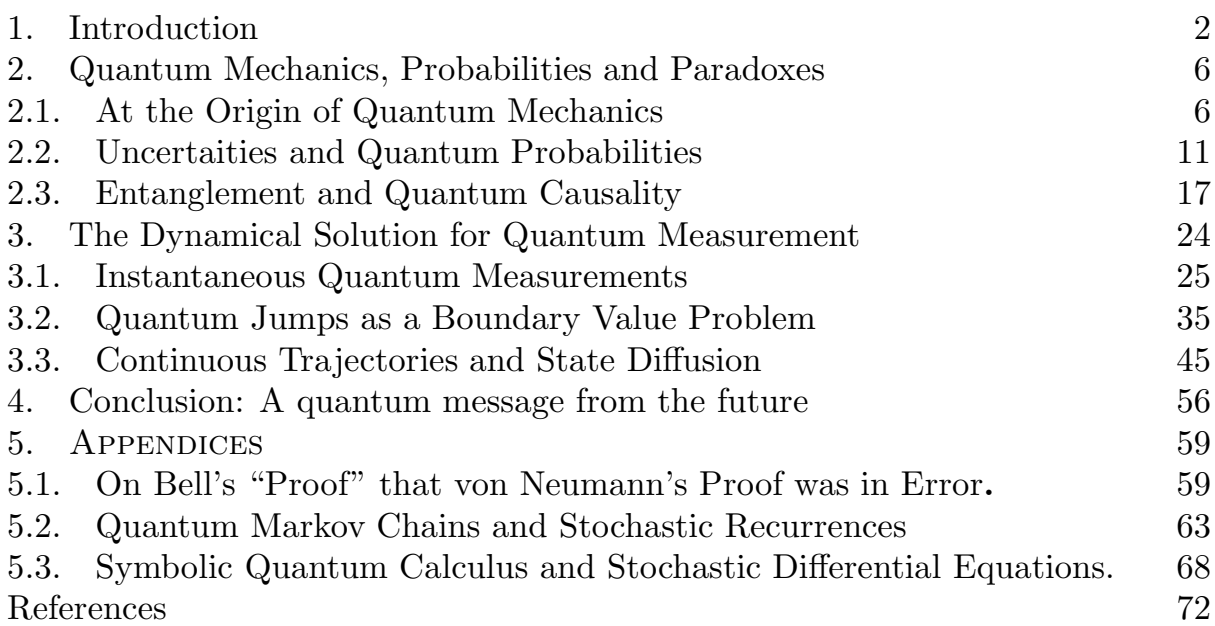

\section{INTRODUCTION}

In 1918 Max Planck was awarded the Nobel Prize in Physics for his quantum theory of blackbody radiation or as we would say now, quantum theory of thermal noise based on the hypothesis of energy discontinuity. Invented in 1900, it inspired an unprecedented revolution in both physical science and philosophy of the 20th century, with an unimaginable deep revision in our way of thinking.

In 1905 Einstein, examining the photoelectric effect, proposed a quantum theory of light, only later realizing that Planck's theory made implicit use of this quantum light hypothesis. Einstein saw that the energy changes in a quantum material oscillator occur in jumps which are multiples of $\omega$. Einstein received Nobel prize in 1922 for his work on the photoelectric effect.

In 1912 Niels Bohr worked in the Rutherford group in Manchester on his theory of the electron in an atom. He was puzzled by the discrete spectra of light which is emitted by atoms when they are subjected to an excitation. He was influenced by the ideas of Planck and Einstein and addressed a certain paradox in his work. How can energy be conserved when some energy changes are continuous and some are discontinuous, i.e. change by quantum amounts? Bohr conjectured that an atom could exist only in a discrete set of stable energy states, the differences of which amount to the observed energy quanta. Bohr returned to Copenhagen and published a revolutionary paper on the hydrogen atom in the next year. He suggested his famous formula

$$
E_{m}-E_{n}=\hbar \omega_{m n}
$$

from which he derived the major laws which describe physically observed spectral lines. This work earned Niels Bohr the 1922 Nobel Prize about $10^{5}$ Swedish Kroner.

Thus before the rise of quantum mechanics 75 years ago, quantum physics had appeared first in the form of quantum stochastics, i. e. the statistics of quantum thermal noise and rules for quantum spontaneous jumps which are often called the "old quantum theory", but in fact the occurrence of the discontinuous quantum 
jumps as individual events have never been explained by the "new" time-continuous quantum mechanics.

Quantum theory is the greatest intellectual achievements of the past century. Since the discovery of quanta by Max Planck exactly 100 years ago on the basis of spectral analysis of quantum thermal noise, and the wave nature of matter, it has produced numerous paradoxes and confusions even in the greatest scientific minds such as those of Einstein, de Broglie, Schrödinger, and it still confuses many contemporary philosophers and scientists who fail to accept the Aristotle's superadditivity law of Nature. Rapid development of the beautiful and sophisticated mathematics for quantum mechanics and the development of its interpretation by Bohr, Born, Heisenberg, Dirac, von Neumann and many others who abandoned traditional causality, were little help in resolving these paradoxes despite the astonishing success in the statistical prediction of the individual quantum phenomena. Both the implication and consequences of the quantum theory of light and matter, as well as its profound mathematical, conceptual and philosophical foundations are not yet understood completely by the majority of quantum physicists.

Specialists in different narrow branches of mathematics and physics rarely understand quantum theory as a common thread which runs through everything. The creators of quantum mechanics, the theory invented for interpretation of the dynamical laws of fundamental particles, were unable to find a consistent interpretation of it since they were physicists with a classical mathematical education. After inventing quantum mechanics they spent much of their lives trying to tackle the Problem of Quantum Measurement, the "only remaining problem" of quantum theory - the problem of its consistent interpretation. Modern quantum phenomenology deals with individual quantum events such as quantum diffusions and jumps which simply do not exist in the orthodox quantum mechanics. There is no place for quantum events neither in the existing quantum field theories nor in the projects of quantum gravity. Moreover, as we shall see, there can't be any, even "hidden variable" solution of the problem of a consistent statistical interpretation of quantum causality in the orthodox quantum theory. But without such interpretation even the unified quantum field theory and gravity would stay 'a thing in itself' as a quantum mechanics of the closed universe without a possibility of any kind of quantum future prediction based on the results of past observations.

In this paper we review the most obscure sides of quantum systems theory which are related to quantum causality and its implications for the time arrow, dynamical irreversibility, consistent histories and prediction of future. These sides traditionally considered by mathematical physicists as 'ill-defined', are usually left to quantum philosophers and theoreticians for vague speculations. Most theoretical physicists have a broad mathematical education, but it tends to ignore two crucial aspects for the solution of these problems - information theory and statistical conditioning. This is why most of theoretical physicists are not familiar with the mathematical development for solving all these questions which has been achieved in quantum probability, information and quantum mathematical statistics during last 25 years. Surely the "professional theoretical physicists ought to be able to do better" (cited from J Bell, [1], p. 173).

As we shall show, the solution to this most fundamental problem of quantum theory can be found in the framework of quantum probability as the rigorous conceptual basis for the algebraic quantum systems theory which is a part of a unified 
mathematics and physics rather than quantum mechanics, and an adequate framework for the treatment of both classical and quantum systems in a unified way. We shall prove that the problem of quantum measurement has a solution in this framework which admits the existence of infinite systems and superselection rules, some of them are already wellused in the algebraic quantum physics. This can be achieved only by imposing a new superselection rule for quantum causality called the nondemolition principle [2] which can be short-frased as 'The past is classical, and it is consistent with the quantum future'. This future-past superselection rule defines the time arrow which leads to a certain restriction of the general relativity principle and the notion of local reality, the profound philosophical implications of which should yet be analyzed. We may conjecture that the difficulties of quantum gravity are fundamentally related to the orthodox point of view that it should be a unification, or consistent with the general relativity and the quantum theory, without taking into account the implications imposed by the causality superselection rule on these theories. In any unified quantum theory with a consistent interpretation addressing modern physics there should be a place for quantum events and Bell's beables as elements of classical reality and quantum causality. Without this it will never be tested experimentally: any experiment deals with the statistics of random events as the only elements of reality, and any reasonable physical theory should admit the predictions based on the statistical causality.

The new infinity and superselection arise with the unitary dilation constructed in this paper for the dynamical derivation of the projection or any other reduction postulate which is simply interpreted as the Bayesian statistical inference for the posterior states based on the prior information in the form of the classical measurement data and the initial quantum state. Such quantum statistical inference called quantum filtering has no conceptual difficulty for the interpretation: it is understood as the prior-posterior transition from quantum possibilities to classical actualities exactly in the same way as it is understood in classical statistics where there is no problem of measurement. Indeed, the collapse of the prior (mixed) state to the posterior (pure) state after the measurement in classical probability is only questioned by those who don't understand that this is purely in the nature of information: it is simply the result of inference due to the gaining information about the existing but a priori unknown pure state. The only distinction of the classical theory from quantum is that the prior mixed states cannot be dynamically achieved from pure initial states without a procedure of either statistical or chaotic mixing. In quantum theory, however, the mixed, or decoherent states can be dynamically induced on a subsystem from the initial pure disentangled states of a composed system simply by a unitary transformation. And the quantum statistical inference (filtering) can result effectively in the "collapse" of a pure initial state to the posterior state corresponding to the result of the measurement (This never happens in classical statistics: a pure prior state induced dynamically by a pure initial state doesn't collapse as it coincide with the posterior pure state as the predetermined result of the inference with no gain of information about the a priori known pure state.)

The main aim of this paper is to give a comprehensive review of the recent progress in this modern quantum theory now known also as quantum stochastics from the historical perspective of the discovery the deterministic quantum evolutions by Heisenberg and Schrödinger to the stochastic evolutions of quantum jumps and quantum diffusion in quantum noise. We will argue that this is the direction in 
which quantum theory would have developed by the founders if the mathematics of quantum stochastics had been discovered by that time. After resolving the typical paradox of the Schrödinger's cat by solving as suggested the related instantaneous quantum measurement problem we move to the dynamical reduction problems for the sequential and time-continuous measurements.

In order to appreciate the mathematical framework and rigorous formulations for resolving these fundamental problems of quantum theory it is quite instructive to start with the typical practical problems of the dynamical systems theory, such as quantum sequential measurements, quantum statistical prediction and quantum feedback control in real time. These problems were indeed set up and analyzed even in the continuous time in the framework of quantum theory first for the linear quantum dynamical systems (quantum open oscillators) over 20 years ago in 3. 4. pioneering the new quantum stochastic approach, and they have been now developed in full generality within this modern rigorous approach to quantum open systems.

We shall concentrate on the modern quantum stochastic approach to quantum consistent histories, quantum trajectories and quantum diffusions originated in 4. 5, 6, 7, 8, 9, 10, 11, 12, 13. In its linear renormalized version this approach gives the output statistics of quantum continuous measurements as a result of the solution of a stochastic differential equation. This allows the direct application of quantum conditioning and filtering methods to tackle the dynamical problem of quantum individual dynamics under continual (trajectorial) measurement. Here we refer mainly to the pioneering and original papers on quantum diffusions in which the relevant quantum structures as mathematical notions and methods were first invented. Most of these results were rigorously proved and published in the mathematical physics literature and is not well known for the larger physical audience.

We shall give a brief account of the relevant mathematics which plays the same role for quantum stochastics as did the classical differential calculus for Newtonian dynamics, and concentrate on its application to the dynamical solution of quantum measurement problems 114, 15, 16, 17, 18, 19, 20], rather than give the full account of all related theoretical papers which use more traditional "down to earth" methods. Among these we would like to mention the papers on quantum decoherence [21, 22], dynamical state reduction program [23, 24], consistent histories and evolutions [25, 26], spontaneous localization and events [27, 28], restricted and unsharp path integrals [29, 30] and their numerous applications to quantum countings, jumps and trajectories in quantum optics and atomic physics [31, 32, 33, 34, 35, 36, 37, 38]. Most of these papers develop a phenomenological approach which is based on a non-Hamiltonian "instrumental" linear master equation giving the statistics of quantum measurements, but is not well adapted for the description of individual and conditional behavior under the continuous measurements. Pearl and Gisin took an opposite, nonlinear, initially even deterministic approach for the individual evolutions, without considering the statistics of measurements [23, 24, 39].

During the 90's many "new" quantum theories appeared in the theoretical and applied physics literature, in particular, the quantum state diffusion theory [40, 41], where the nonlinear quantum filtrering stochastic equations for diffusive measurements have been used without even a reference to the continuous measurements, and quantum trajectories in quantum optics 442, 43, 44, 45, 47, 46, where the stochastic solutions to quantum jump equations have been constructed even without a reference to quantum stochastic filtering equations. However the transition from 
nonlinear to linear stochastic equations and the quantum stochastic unitary models for the underlying Hamiltonian microscopic evolutions remain unexplained in these papers. Moreover, most of these papers claim primarity or universality of the stochastic evolution but treat very particular phenomenological models which are based only on the counting, or sometimes diffusive (homodyne and heterodyne) models of quantum noise and output process, and reinvent many notions such as quantum conditioning and adaptedness with respect to the individual trajectories, without references to the general quantum stochastic measurement and filtering (conditioning) theory. An exception occurred only in [47, 48], where our quantum stochastic filtering theory which had been developed for these purposes in the 80's, was well understood both at a macroscopic and microscopic level. This explains why a systematic review of this kind is needed.

In order to appreciate the quantum drama which has been developing through the whole century, it seems useful to give a account of the discovery of quantum mechanics and its probabilistic interpretation at the 20th of the past century. This is briefly done in the first sections Chapter 1. More about the discovery of the "old" and "new" quantum mechanics starting from the Plank's quanta 49 and thei relation to the modern theory of quantum noise and applications to quantum bits one can find in 50]. Readers who are not interested in the historical perspective of this subject and paradoxes of its interpretation are advised to start with the Chapter 2 dealing with the famous problem of quantum measurement. The specialists who are familiar with this might still find intersting a review on quantum stochastics, causality, consistent trajectories, continual measurements, quantum jumps and diffusions, and will find the origin and explanation of these modern quantum theories in the last sections.

\section{Quantum Mechanics, Probabilities and Paradoxes}

If anyone says he can think about quantum problems without getting giddy, that only shows he has not understood the first thing about them - Max Planck.

\subsection{At the Origin of Quantum Mechanics.}

The whole is more than the sum of its parts - Aristotle.

This is the famous superadditivity law from Aristotle's Metaphysics which studies 'the most general or abstract features of reality and the principles that have universal validity'. Certainly in this broad definition quantum physics is the most essential part of metaphysics.

Quantum theory is a mathematical theory which studies the most fundamental features of reality in a unified form of waves and matter, it raises and solves the most fundamental riddles of Nature by developing and utilizing mathematical concepts and methods of all branches of modern mathematics, including probability and statistics. Indeed, it began with the discovery of new laws for 'quantum' numbers, the natural objects which are the foundation of pure mathematics. ('God made the integers; the rest is man's work' - Kronecker). Next it invented new applied mathematical methods for solving quantum mechanical matrix and partial differential equations. Then it married probability with algebra to obtain unified treatment of waves and particles in nature, giving birth to quantum probability and creating new 
branches of mathematics such as quantum logics, quantum topologies, quantum geometries, quantum groups. It inspired the recent creation of quantum analysis and quantum calculus, as well as quantum statistics and quantum stochastics.

2.1.1. The discovery of matrix mechanics. In 1925 a young German theoretical physicist, Heisenberg, gave a preliminary account of a new and highly original approach to the mechanics of the atom [51]. He was influenced by Niels Bohr and proposed to substitute for the position coordinate of an electron in the atom complex arrays

$$
q_{m n}(t)=q_{m n} e^{i \omega_{m n} t}
$$

oscillating with Bohr's frequencies $\omega_{m n}=\hbar^{-1}\left(E_{m}-E_{n}\right)$. He thought that they would account for the random jumps $E_{m} \mapsto E_{n}$ in the atom corresponding to the spontaneous emission of the energy quanta $\hbar \omega_{m n}$. His Professor, Max Born, was a mathematician who immediately recognized an infinite matrix algebra in Heisenberg's multiplication rule for the tables $\mathrm{Q}(t)=\left[q_{m n}(t)\right]$. The classical momentum was also replaced by a similar matrix,

$$
\mathrm{P}(t)=\left[p_{m n} e^{i \omega_{m n} t}\right],
$$

as the Planck's electro- magnetic quanta were thought being induced by oscillator equations

$$
\frac{\mathrm{d}}{\mathrm{d} t} q_{m n}(t)=i \omega_{m n} q_{m n}(t), \frac{\mathrm{d}}{\mathrm{d} t} p_{m n}(t)=i \omega_{m n} p_{m n}(t) .
$$

These equations written in terms of the matrix algebra as

$$
\frac{\mathrm{d}}{\mathrm{d} t} \mathrm{Q}(t)=\frac{i}{\hbar}[\mathrm{H}, \mathrm{Q}(t)], \frac{\mathrm{d}}{\mathrm{d} t} \mathrm{P}(t)=\frac{i}{\hbar}[\mathrm{H}, \mathrm{P}(t)],
$$

now are known as the Heisenberg equations, where $\mathrm{H}$ called the Hamiltonian, is the diagonal matrix $\mathrm{E}=\left[E_{n} \delta_{m n}\right]$, and $[\mathrm{H}, \mathrm{B}]$ denotes the matrix commutator $\mathrm{HB}-\mathrm{BH}$. In order to achieve the correspondence with the classical mechanics, their young colleague Jordan suggested to postulate the canonical (Heisenberg) commutation relations

$$
[\mathrm{Q}(t), \mathrm{P}(t)]=i \hbar \mathrm{I},
$$

where $\mathrm{I}$ is the unit matrix $\left[\delta_{m n}\right]$, 52]. This made the equations (2.1) formally equivalent to the Hamiltonian equations

$$
\frac{\mathrm{d}}{\mathrm{d} t} \mathrm{Q}(t)=H_{p}(\mathrm{Q}(t, \mathrm{P}(t))), \frac{\mathrm{d}}{\mathrm{d} t} \mathrm{P}(t)=-H_{q}(\mathrm{Q}(t), \mathrm{P}(t)),
$$

but the derivatives $H_{p}, H_{q}$ of the Hamiltonian function $H(q, p)$ were now replaced by the appropriate matrix-algebra functions of the noncommuting $\mathrm{P}$ and $\mathrm{Q}$ such that $H(\mathrm{Q}, \mathrm{P})=\mathrm{H}$. For the non-relativistic electron in a potential field $\phi$ this yielded the Newton equation

$$
m \frac{\mathrm{d}^{2}}{\mathrm{~d} t^{2}} \mathrm{Q}(t)=-\nabla \phi(\mathrm{Q}(t)),
$$

but with the non-commuting initial conditions $\mathrm{Q}(0)$ and $\frac{\mathrm{d}}{\mathrm{d} t} \mathrm{Q}(0)=\frac{1}{m} \mathrm{P}(0)$, where the potential force is replaced by the corresponding matrix function of $\mathrm{Q}=\left[q_{m n}\right]$.

Thus the new, quantum mechanics was first invented in the form of matrix mechanics, emphasizing the possibilities of quantum transitions, or jumps between the stable energy states $E_{n}$ of an electron. However there was no mechanism suggested 
to explain the actualities of these spontaneous transitions in the continuous time. In 1932 Heisenberg was awarded the Nobel Prize for his pioneering work on the mathematical formulation of the new physics.

Conceptually, the new atomic theory was based on the positivism of Mach as it operated not with real space-time but with only observable quantities like atomic transitions. However many leading physicists were greatly troubled by the prospect of loosing reality and deterministic causality in the emerging quantum physics. Einstein, in particular, worried about the element of 'chance' which had entered physics. In fact, this worries came rather late since Rutherford had introduced a spontaneous effect when discussing radio-active decay in 1900.

2.1.2. The discovery of wave mechanics. In 1923 de Broglie, inspired by the works of Einstein and Planck, extended the wave-corpuscular duality also to material particles. He used the Hamilton-Jacobi theory which had been applied both to particles and waves. In 1928 de Broglie received the Nobel Prize for this work.

In 1925, Schrödinger gave a seminar on de Broglie's material waves, and a member of the audience suggested that there should be a wave equation. Within a few weeks Schrödinger found his celebrated wave equation, first in a relativistic, and then in the non-relativistic form [53]. Instead of seeking the classical solutions to the Hamilton-Jacobi equation

$$
H\left(q, \frac{\hbar}{i} \frac{\partial}{\partial q} \ln \psi\right)=E
$$

he suggested finding those wave functions $\psi(q)$ which satisfy the linear equation

$$
H\left(q, \frac{\hbar}{i} \frac{\partial}{\partial q}\right) \psi=E \psi
$$

(It coincides with the former equation only if the Hamiltonian function $H(q, p)$ is linear with respect to $p$ but not for the non-relativistic $\left.H(q, p)=\frac{1}{2 m} p^{2}+\phi(q)\right)$.

Schrödinger published his revolutionary wave mechanics in a series of six papers 54 in 1926 during a short period of sustained creative activity that is without parallel in the history of science. Like Shakespeare, whose sonnets were inspired by a dark lady, Schrödinger was inspired by a mysterious lady of Arosa where he took ski holidays during the Christmas 1925 but 'had been distracted by a few calculations'. This was the second formulation of the new quantum theory, which he successfully applied to the Hydrogen atom, oscillator and other quantum mechanical systems, solving the corresponding Sturm-Liouville boundary-value problems of mathematical physics. The mathematical equivalence between the two formulations of quantum mechanics was understood by Schrödinger in the fourth paper where he suggested the non-stationary wave equation written in terms of the Hamiltonian operator $\mathrm{H}=H\left(q, \frac{\hbar}{i} \frac{\partial}{\partial q}\right)$ for the complex time-dependent wave-function $\psi(t, q)$ simply as

$$
i \hbar \frac{\partial}{\partial t} \psi(t)=\mathrm{H} \psi(t)
$$

and he also introduced operators associated with each dynamical variable.

Unlike Heisenberg and Born's matrix mechanics, the general reaction towards wave mechanics was immediately enthusiastic. Plank described Schrödinger's wave mechanics as 'epoch-making work'. Einstein wrote: 'the idea of your work springs from true genius...'. Next year Schrödinger was nominated for the Nobel Prize, but 
he failed to receive it in this and five further consecutive years of his nominations by most distinguished physicists of the world, the reason behind his rejection being 'the highly mathematical character of his work'. Only in 1933 did he receive his prize, this time jointly with Dirac, and this was the first, and perhaps the last, time when the Nobel Prize for physics was given to true mathematical physicists.

Following de Broglie, Schrödinger initially thought that the wave function corresponds to a physical vibration process in a real continuous space-time because it was not stochastic, but he was puzzled by the failure to explain the blackbody radiation and photoelectric effect from this wave point of view. In fact the wave interpretation applied to light quanta leads back to classical electrodynamics, as his relativistic wave equation for a single photon coincides mathematically with the classical wave equation. However after realizing that the time-dependent $\psi$ in (2.5) must be a complex function, he admitted in his fourth 1926 paper 54 that the wave function $\psi$ cannot be given a direct interpretation, and described the wave density $|\psi|^{2}=\psi \bar{\psi}$ as a sort of weight function for superposition of point-mechanical configurations.

Although Schrödinger was a champion of the idea that the most fundamental laws of the microscopic world are absolutely random even before he discovered wave mechanics, he failed to see the probabilistic nature of $\psi \bar{\psi}$. Indeed, his equation was not stochastic, and it didn't account for the individual random jumps $E_{m} \rightarrow E_{n}$ of the Bohr-Heisenberg theory but rather opposite, it did prescribe the preservation of the eigenvalues $E=E_{n}$.

For the rest of his life Schrödinger was trying to find apparently without a success a more fundamental equation which would be responsible for the energy transitions in the process of measurement of the quanta $\hbar \omega_{m n}$. As we shall see, he was right assuming the existence of such equation.

2.1.3. Interpretations of quantum mechanics. The creators of the rival matrix quantum mechanics were forced to accept the simplicity and beauty of Schrödinger's approach. In 1926 Max Born put forward the statistical interpretation of the wave function by introducing the statistical means

$$
\langle\mathrm{X}\rangle=\int \bar{\psi}(x) x \psi(x) \mathrm{d} x
$$

for Hermitian dynamical variables $\mathrm{X}$ in the physical state, described in the eigenrepresentation of $\mathrm{X}$ by a complex function $\psi(x)$ normalized as $\langle\mathrm{I}\rangle=1$. Thus he identified the quantum states with one-dimensional subspaces of a Hilbert space $\mathcal{H}$ corresponding to the normalized $\psi$ defined up to a complex factor $\mathrm{e}^{i \theta}$. This was developed in Copenhagen and gradually was accepted by almost all physicists as the "Copenhagen interpretation". Born by education was a mathematician, but he received the Nobel Prize in physics for his statistical studies of wave functions later in 1953 as a Professor of Natural Philosophy at Edinburgh. Bohr, Born and Heisenberg considered electrons and quanta as unpredictable particles which cannot be visualized in the real space and time.

The most outspoken opponent of a/the probabilistic interpretation was Einstein. Albert Einstein admired the new development of quantum theory but was suspicious, rejecting its acausality and probabilistic interpretation. It was against his scientific instinct to accept statistical interpretation of quantum mechanics as a complete description of physical reality. There are famous sayings of his on that account: 
'God is subtle but he is not malicious', 'God doesn't play dice'

During these debates on the probabilistic interpretation of quantum mechanics of Einstein between Niels Bohr, Schrödinger often sided with his friend Einstein, and this may explain why he was distancing himself from the statistical interpretation of his wave function. Bohr invited Schrödinger to Copenhagen and tried to convince him of the particle-probabilistic interpretation of quantum mechanics. The discussion between them went on day and night, without reaching any agreement. The conversation, however deeply affected both men. Schrödinger recognized the necessity of admitting both wave and particles, but he never devised a comprehensive interpretation rival to Copenhagen orthodoxy. Bohr ventured more deeply into philosophical waters and emerged with his concept of complementarity:

\section{Evidence obtained under different experimental conditions cannot be comprehended within a single picture, but must be regarded as comple- mentary in the sense that only the totality of the phenomena exhausts the possible information about the objects.}

In his later papers Schrödinger accepted the probabilistic interpretation of $\psi \bar{\psi}$, but he did not consider these probabilities classically, instead he interpreted them as the strength of our belief or anticipation of an experimental result. In this sense the probabilities are closer to propensities than to the frequencies of the statistical interpretation of Born and Heisenberg. Schrödinger had never accepted the subjective positivism of Bohr and Heisenberg, and his philosophy is closer to that called representational realism. He was content to remain a critical unbeliever.

There have been many other attempts to retain the deterministic realism in the quantum world, the most extravagant among these being the ensemble-world interpretations of Bohm 56] and Everett [57]. The first interpretational theory, known as the pilot-wave theory, is based on the conventional Schrödinger equation which is used to define the flow of a classical fluid in the configuration space. The predictions of this classical macroscopic theory coincide with the statistical predictions of the orthodox quantum theory only for the ensembles of coordinate-like observables with the initial probability distribution over the many worlds given by the initial pilot wave. Other observables like momenta which are precisely determined at each point by the velocity of this fluid, have no uncertainty under the fixed coordinates. This is inconsistent with the prediction of quantum theory for individual systems, and there is no way to incorporate the stochastic dynamics of sequentially monitored individual quantum particles in the single world into this fluid dynamics. Certainly this is a variation of the de Broglie-Schrödinger old interpretation, and it doesn't respect the Bell's first principle for the interpretational theories "that it should be possible to formulate them for small systems" [1], p. 126.

The Everett's many-world interpretation also assumes that the classical configurations at each time are distributed in the comparison class of possible worlds worth probability density $\psi \bar{\psi}$. However no continuity between present and past configurations is assumed, and all possible outcomes of their measurement are realized every time, each in a different edition of the continuously multiplying universe. The observer in a given brunch of the universe is aware only of what is going on in that particular branch, and this results in the reduction of the wave-function. This would be macroscopically equivalent to the pilot-wave theory if the de BroglieSchrödinger-Bohm fluid dynamics could be obtained as the average of wave equations over all brunches. An experienced statistician would immediately recognize 
in this many-world interpretation an ensemble model for a continuously branching stochastic process, and would apply the well-developed stochastic analysis and differential calculus to analyze this dynamical model. However no stochastic equation for a continuously monitored branch was suggested in this theory, although there should be many if at all, corresponding to many possible choices of classical configurations (e.g. positions or momenta) for a single many-world Schrödinger equation.

Living simultaneously in many worlds would have perhaps certain advatages, but from the philosophical and practical point of view, however, to have an infinite number (continuum product of continua?) of real worlds at the same time without their communication seems not better than to have none. As Bell wrote in [1], p. 134: "to have multiple universes, to realize all possible configurations of particles, would have seemed grotesque". Even if such a weighted many-world dynamical theory had been developed to a satisfactory level, it would have been reformulated in terms of well-established mathematical language as a stochastic evolutionary theory in the single world with the usual statistical interpretation. In fact, the stochastic theory of continuously observed quantum systems has been already derived, not just developed, in full generality and rigor in quantum stochastics, and it will be presented in the last sections. But first we shall demonstrate the underlying ideas on the elementary single-transition level.

\subsection{Uncertaities and Quantum Probabilities.}

In mathematics you don't understand things. You just get used to them - John von Neumann.

In 1932 von Neumann put quantum theory on firm theoretical basis by setting the mathematical foundation for new, quantum, probability theory, the quantitative theory for counting non commuting events of quantum logics. This noncommutative probability theory is based on essentially more general axioms than the classical (Kolmogorovian) probability of commuting events, which form common sense Boolean logic, first formalized by Aristotle. The main idea of this theory is based upon the empirical fact that the maximal number of alternatives in each experiment over a quantum system is smaller than the quantum probability dimensionality of the system, i.e. the dimensionality of the linear space of all propensities, or empirical frequencies defining the quantum state. Unlike in the classical world were the maximal number of alternatives always coincides with the dimensionality of the probability space. Actually the quantum dimensionality squares the classical one, is the sum of such squares for the hybrid systems, and this defines every quantum probability space as the space of density matrices rather than space of density functions.

Quantum probability has been under extensive development during the last 30 years since the introduction of algebraic and operational approaches for treatment of noncommutative probabilities, and currently serves as the mathematical basis for quantum stochastics and information theory. Unfortunately its recent development was more in parallel with classical probability theory rather than with physics, and many mathematical technicalities of quantum calculi prevented its acceptance in physics.

In the next section we shall demonstrate the main ideas of quantum probability arising from the application of classical probability theory to quantum phenomena 
on the simple quantum systems. The most recent mathematical development of these models and methods leads to a profound stochastic theory of quantum open systems with many applications including quantum information, quantum measurement, quantum filtering and prediction and quantum feedback control, some of them are presented in the last sections.

2.2.1. Heisenberg uncertainty relations. In 1927 Heisenberg derived [58] his famous uncertainty relations

$$
\Delta \mathrm{Q} \Delta \mathrm{P} \geq \hbar / 2, \quad \Delta \mathrm{T} \Delta \mathrm{E} \geq \hbar / 2
$$

which gave mathematical support to the revolutionary complementary principle of Bohr. As Dirac stated:

Now when Heisenberg noticed that, he was really scared.

The first relation can be easily understood in the Schrödinger representations $\mathrm{Q}=x, \mathrm{P}=\frac{\hbar}{i} \frac{\partial}{\partial x}$ in terms of the standard deviations

$$
\Delta \mathrm{Q}=\left\langle\widetilde{\mathrm{Q}}^{2}\right\rangle^{1 / 2}, \quad \Delta \mathrm{P}=\left\langle\widetilde{\mathrm{P}}^{2}\right\rangle^{1 / 2}
$$

where $\widetilde{\mathrm{Q}}=\mathrm{Q}-\langle\mathrm{Q}\rangle \mathrm{I}$ and $\widetilde{\mathrm{P}}=\mathrm{P}-\langle\mathrm{P}\rangle \mathrm{I}$ have the same commutator $[\widetilde{\mathrm{Q}}, \widetilde{\mathrm{P}}]=\frac{\hbar}{i} \mathrm{I}$ as $\mathrm{Q}$ and $\mathrm{P}$. To this end one can use the Schwarz inequality $\left\langle\widetilde{\mathrm{Q}}^{2}\right\rangle\left\langle\widetilde{\mathrm{P}}^{2}\right\rangle \geq|\langle\widetilde{\mathrm{Q}} \widetilde{\mathrm{P}}\rangle|^{2}$ and that

$$
|\langle\widetilde{\mathrm{Q}} \widetilde{\mathrm{P}}\rangle| \geq|\operatorname{Im}\langle\widetilde{\mathrm{Q}} \widetilde{\mathrm{P}}\rangle|=\frac{1}{2}|\langle[\widetilde{\mathrm{Q}}, \widetilde{\mathrm{P}}]\rangle|=\frac{\hbar}{2}
$$

The second uncertainty relation, which was first stated by analogy of $t$ with $x$ and of $\mathrm{E}$ with $\mathrm{P}$, cannot be proved in the same way as the time operator $\mathrm{T}$ does not exist in the Schrödinger's Hilbert space $\mathcal{H}$ of wave functions $\psi(x)$. So, it is usually interpreted in terms of the time $\Delta \mathrm{T}$ required for a quantum nonstationary state with spread in energy $\Delta \mathrm{E}$ to evolve to an orthogonal and hence distiguishable state [59]. However it can also be proved [60, 61] in terms of the standard deviation $\Delta \mathrm{T}$ of the optimal statistical estimate for the time $t$ of the wave packet in the Schrödinger's picture $\psi(t-s)$ with respect to an unknown initial $t_{0}=s$, with the energy spread $\Delta \mathrm{E}$. The similar problem for the shift parameter $q$ in the wave packet $\psi(x-q)$ defines the optimal estimate as the measurement of the coordinate operator $\mathrm{Q}=x$. Although the optimal estimation of $t$ cannot be treated as the usual quantum mechanical measurement of a self-adjoint operator in $\mathcal{H}$, the optimal estimation can been realized [62] by the measurement of the self-adjoint operator $\mathrm{T}=s$ in an extended (doubled) Hilbert state space $\mathbb{H}=\mathcal{H} \oplus \mathcal{H}$ of all the functions

$$
\Psi(t, s)=\psi(t-s) \oplus \overline{\varphi(t-s)}=\Psi(0, s-t), \quad \psi, \varphi \in \mathcal{H} .
$$

Note that such extension is simply a new reducible representation (time representation) of the quantum system in which the Hamiltonian $\mathrm{H}$ is the momentum operator $\mathrm{E}=\frac{\hbar}{i} \frac{\partial}{\partial s}$ along the time coordinate $s$ if the initial states $\Psi(0)$ are restricted to the embedded subspace $\mathcal{H}$ by the initial data constraint $\Psi(0, s)=\psi(-s)(\varphi=0)$. This subspace is not invariant under the (measurement of) $\mathrm{T}$, and after this measurement it should be projected back onto $\mathcal{H}$.

Einstein launched an attack on the uncertainty relation at the Solvay Congress in 1927 , and then again in 1930, by proposing cleverly devised thought experiments 
which would violate this relation. Most of these imaginary experiments were designed to show that interaction between the microphysical object and the measuring instrument is not so inscrutable as Heisenberg and Bohr maintained. He suggested, for example, a box filled with radiation with a clock described by the pointer coordinate $x$. The clock is designed to open a shutter and allow one photon to escape at the time T. He argued that the time of escape and the photon energy E can both be measured with arbitrary accuracy by measuring the pointer coordinate and by weighing the box before and after the escape as the difference of the weights $y$.

After proposing this argument Einstein is reported to have spent a happy evening, and Niels Bohr an unhappy one. However after a sleepless night Bohr showed next morning that Einstein was wrong. Mathematically Bohr's explanation of the Einstein experiment can be expressed as the usual measurement of two compatible variables $x$ and $y$ of the of the total system under the question by the following simple'signal plus noise' formula

$$
X=s+Q, \quad Y=\frac{\hbar}{i} \frac{\partial}{\partial s}-P,
$$

where $Q$ and $P$ are the position and momentum operators of the compensation weight under the box. Here the measuring quantity $X$, the pointer coordinate of the clock, realizes an unsharp measurement of the self-adjoint time operator $\mathrm{T}=s$ representing the time in the extended Hilbert space $\mathbb{H}$, and the observable $Y$ realizes the indirect measurement of photon energy $\mathrm{E}=i \hbar \frac{\partial}{\partial s}$ in $\mathbb{H}$. Due to the initial independence of the weight, the commuting observables $X$ and $Y$ in the Einstein experiment will have even greater uncertainty

$$
\Delta X \Delta Y=\Delta \mathrm{T} \Delta \mathrm{E}+\Delta Q \Delta P \geq \hbar
$$

than that predicted by Heisenberg uncertainty $\Delta \mathrm{T} \Delta \mathrm{E} \geq \hbar / 2$ as it is the sum with $\Delta Q \Delta P \geq \hbar / 2$. This uncertainty remains obviously valid if the states $\psi \in \mathbb{H}$ of the 'extended photon' are restricted to only physical photon states $\psi \in \mathcal{H}$ corresponding to the positive spectrum of $\mathrm{E}$.

2.2.2. Nonexistence of hidden variables. Einstein hoped that eventually it would be possible to explain the uncertainty relations by expressing quantum mechanical observables as functions of some hidden variables $\lambda$ in deterministic physical states such that the statistical aspect will arise as in classical statistical mechanics by averaging these observables over $\lambda$.

Von Neumann's monumental book 63 on the mathematical foundations of quantum theory was therefore a timely contribution, clarifying, as it did, this point. Inspired by Lev Landau, he introduced, for the unique characterization of the statistics of a quantum ensemble, the statistical density operator $\rho$ which eventually, under the name normal, or regular state, became a major tool in quantum statistics. He considered the linear space $\mathfrak{L}$ of all bounded Hermitian operators $\mathrm{L}=\mathrm{L}^{\dagger}$ as potential observables in a quantum system described by a Hilbert space $\mathbb{H}$ of all normalizable wave functions $\psi$. Although von Neumann considered any complete inner product complex linear space as the Hilbert space, it is sufficient to reproduce his analysis for a finite-dimensional $\mathbb{H}$. He defined the expectation $\langle\mathrm{L}\rangle$ of each $\mathrm{L} \in \mathfrak{L}$ in a state $\rho$ by the linear functional $\mathrm{L} \mapsto\langle\mathrm{L}\rangle$ of the regular form $\langle\mathrm{L}\rangle=\operatorname{Tr} \mathrm{L} \rho$, where $\operatorname{Tr}$ denotes the linear operation of trace applied to the product of all operators on the right. He noted that in order to have positive probabilities for the potential quantum mechanical events $E$ as the expectations $\langle E\rangle$ of yes-no 
observables described by the Hermitian projectors $\mathrm{E} \in \mathfrak{L}$ (i.e. with $\{0,1\}$ spectrum), and probability one for the identity event $I=1$ described by the identity operator I ,

$$
\operatorname{Pr}\{E=1\}=\operatorname{Tr} E \rho \geq 0, \quad \operatorname{Pr}\{I=1\}=\operatorname{Tr} \rho=1,
$$

the statistical operator $\rho$ must be positive-definite and have trace one. Then he proved that any linear (and even any additive) physically continuous functional $\mathrm{L} \mapsto$ $\langle\mathrm{L}\rangle$ is regular, i.e. has such trace form. He applied this technique to the analysis of the completeness problem of quantum theory, i.e. whether it constitutes a logically closed theory or whether it could be reformulated as an entirely deterministic theory through the introduction of hidden parameters (additional variables which, unlike ordinary observables, are inaccessible to measurements). He came to the conclusion that

the present system of quantum mechanics would have to be objectively false, in order that another description of the elementary process than the statistical one may be possible

(quoted on page 325 in [63])

To prove this theorem, von Neumann showed that there is no such state which would be dispersion-free simultaneously for all possible quantum events $E \in \mathfrak{L}$ described by all Hermitian projectors $E^{2}=E$. For each such state, he argued,

$$
\left\langle E^{2}\right\rangle=\langle E\rangle=\langle E\rangle^{2}
$$

for all such $E$ would imply that $\rho=\mathrm{O}$ (O denotes the zero operator) which cannot be statistical operator as $\operatorname{TrO}=0 \neq 1$. Thus no state can be considered as a mixture of dispersion-free states, each of them associated with a definite value of hidden parameters. There are simply no such states, and thus, no hidden parameters. In particular this implies that the statistical nature of pure states, which are described by one-dimensional projectors $\rho=P_{\psi}$ corresponding to wave functions $\psi$, cannot be removed by supposing them to be a mixture of dispersion-free substates.

It is widely believed that in 1966 John Bell showed that von Neuman's proof was in error, or at least his analysis left the real question untouched [64. To discredit the von Neumann's proof he constructed an example of dispersion-free states parametrized for each quantum state $\rho$ by a real parameter $\lambda$ for a simplest quantum system corresponding to the two dimensional $\mathbb{H}=\mathfrak{h}$ (we shall use the little $\mathfrak{h} \simeq \mathbb{C}^{2}$ for this simplest state space and Pauli matrix calculus in notation of the Appendix 1). He succeeded to do this by weakening the assumption of the additivity for such states, requiring it only for the commuting observables in $\mathfrak{L}$, and by abandoning the linearity of the constructed expectations in $\rho$. There is no reason, he argued, to keep the linearity in $\rho$ for the observable eigenvalues determined by $\lambda$ and $\rho$, and to demand the additivity for non-commuting observables as they are not simultaneously measurable: The measured eigenvalues of a sum of noncommuting observables are not the sums of the eigenvalues of this observables measured separately. For each spin-operator $\mathrm{L}=\sigma(\mathbf{l})$ given by a 3 -vector $\mathbf{l} \in \mathbb{R}^{3}$ as in the Appendix 1 Bell found a discontinuous family $s_{\lambda}$ (l) of dispersion-free values $\pm l$, $l=|\mathbf{l}|$, parameterized by $|\lambda| \leq 1 / 2$, which reproduce the expectation $\langle\sigma(\mathbf{l})\rangle=\mathbf{l} \cdot \mathbf{r}$ in the pure quantum state described by a unit polarization vector $\mathbf{r}$ in $\mathbb{R}^{3}$ when uniformly averaged over the $\lambda$.

Although the Bell's analysis of the von Neumann theorem is mathematically incomplete as it ignores physical continuity which was assumed by von Neumann 
in his definition of physical states, this is not the main reason for failure of the Bell's. The reason for failure of the Bell's and others hidden variable arguments is given in the Appendix 1 where is shown that all dispersion free states even if they existed, not just the one constructed by Bell for the exceptional case $\operatorname{dim} \mathfrak{h}=2$, cannot be extended to the quantum composed systems. All hidden variable theories are incompatible with quantum composition principle which multiples the dimensionally of the Hilbert space by the dimensionality of the state-vector space of the additional quantum system. In higher dimensions of $\mathbb{H}$ all such irregular states are ruled out by Gleason's theorem [65] who proved that there is no even one additive zero-one value probability function if $\operatorname{dim} \mathbb{H}>2$. In order that a hidden variable description of the elementary quantum process may be possible, the present postulates of quantum mechanics such as the composition principle would have to be objectively false.

2.2.3. Complementarity and common sense. In view of the decisive importance of this analysis for the foundations of quantum theory, Birkhoff and von Neumann [66] setup a system of formal axioms for the calculus of logico-theoretical propositions concerning results of possible measurements in a quantum system described by a Hilbert space $\mathbb{H}$. They started by formalizing the calculus of quantum propositions corresponding to the potential idealized events $E$ described by orthoprojectors in $\mathbb{H}$, the projective operators $E=E^{2}$ which are orthogonal to their complements $E^{\perp}=\mathrm{I}-E$ in the sense $E^{\dagger} E^{\perp}=\mathrm{O}$, where $\mathrm{O}$ denotes the multiplication by 0 . The set $\mathcal{P}(\mathbb{H})$ of all othoprojectors, equivalently defined by

$$
E^{\dagger}=E^{\dagger} E=E,
$$

is the set of all Hermitian projectors $E \in \mathfrak{L}$ as the only observables with two eigenvalues $\{1,0\}$ ("yes" and "no"). Such calculus coincides with the calculus of linear subspaces $\mathfrak{e} \subseteq \mathbb{H}$ including 0 -dimensional subspace $\mathbb{O}$, in the same sense as the common sense propositional calculus of classical events coincides with the calculus in a Boolean algebra of subsets $E \subseteq \Omega$ including empty subset $\varnothing$. The subspaces $\mathfrak{e}$ are defined by the condition $\mathfrak{e}^{\perp \perp}=\mathfrak{e}$, where $\mathfrak{e}^{\perp}$ denotes the orthogonal complement $\{\phi \in \mathbb{H}:\langle\phi \mid \psi\rangle=0, \psi \in \mathfrak{e}\}$ of $\mathfrak{e}$, and they uniquely define the propositions $E$ as the orthoprojectors $P(\mathfrak{e})$ onto the ranges

$$
\mathfrak{e}=\operatorname{range} E:=E \mathbb{H}
$$

of $E \in \mathcal{P}(\mathbb{H})$. In this calculus the logical ordering $E \leq F$ implemented by the algebraic relation $E F=E$ coincides with

$$
\text { range } E \subseteq \text { range } F,
$$

the conjunction $E \wedge F$ corresponds to the intersection,

$$
\text { range }(E \wedge F)=\operatorname{range} E \cap \operatorname{range} F,
$$

however the disjunction $E \vee F$ is represented by the linear sum $\mathfrak{e}+\mathfrak{f}$ of the corresponding subspaces but not their union

$$
\text { range } E \cup \operatorname{range} F \subseteq \text { range }(E \vee F),
$$

and the smallest orthoprojector $\mathrm{O}$ corresponds to zero-dimensional subspace $\mathbb{O}=$ $\{0\}$ but not the empty subset $\varnothing$ (which is not linear subspace). Note that although range $(E+F)=\mathfrak{e}+\mathfrak{f}$ for any $E, F \in \mathcal{P}(\mathbb{H})$, the operator $E+F$ is not the orthoprojector $E \vee F$ corresponding to $\mathfrak{e}+\mathfrak{f}$ unless $E F=O$. This implies that the distributive law characteristic for propositional calculus of classical logics no 
longer holds. However it still holds for the compatible propositions described by commutative orthoprojectors due to the orthomodularity property

$$
E \leq I-F \leq G \Longrightarrow(E \vee F) \wedge G=E \vee(F \wedge G)
$$

Actually as we shall see, a propositions $E$ can become an event if and only if it may serve as a condition for any other proposition $F$. In terms of the natural order of the propositions this can be written as

$$
E^{\perp} \wedge F=\mathrm{I}-E \wedge F \quad \forall F
$$

where $E^{\perp}=\mathrm{I}-E$, and it is equivalent to the compatibility $E F=F E$ of the event-orthoprojector $E$ with any other orthoprojector $F$ of the system.

For each regular state corresponding to a density operator $\rho$, one can obtain the probability function $\langle E\rangle=\operatorname{Tr} E \rho$ on $\mathcal{P}(\mathbb{H})$ called the quantum probability measure. It can also be defined as a function

$$
\mathrm{P}(\mathfrak{e})=\operatorname{Pr}\{P(\mathfrak{e})=1\}=\langle P(\mathfrak{e})\rangle
$$

on the set $\mathcal{E}$ of all subspaces $\mathfrak{e}$ of $\mathbb{H}$. It is obviously positive, $\mathrm{P}(\mathfrak{e})>0$, with $\mathrm{P}(\mathbb{O})=0$, normalized, $\mathrm{P}(\mathbb{H})=1$, and additive but only for orthogonal $\mathfrak{e}$ and $\mathfrak{f}$ :

$$
\mathfrak{e} \perp \mathfrak{f} \Rightarrow P(\mathfrak{e}+\mathfrak{f})=P(\mathfrak{e})+P(\mathfrak{f}) .
$$

These properties are usually taken as definition of a probabilistic state on the quantum logic $\mathcal{E}$. Note that not any ortho-additive function $\mathrm{P}$ is a priori regular, i.e. induced by a density operator $\rho$ on $\mathcal{E}$ as $\mathrm{P}(\mathfrak{e})=\operatorname{Tr} P(\mathfrak{e}) \rho$. However Gleason proved 65 that every ortho-additive normalized function $\mathcal{E} \rightarrow[0,1]$ is regular in this sense if $2<\operatorname{dim} \mathbb{H}<\infty$ (he proved this also for the case $\operatorname{dim} \mathbb{H}=\infty$ under the natural assumption of countable ortho-additivity, and it is also true for $\operatorname{dim} \mathbb{H}=2$ under the quantum composition assumption, see the Appendix 1). Any statistical mixture of such (regular) probability functions is obviously a (regular) probability function, and the extreme functions of this convex set correspond to the pure (regular) states $\rho=\psi \psi^{\dagger}$.

Two propositions $E, F$ are called complementary if $E \vee F=\mathrm{I}$, orthocomplementary if $E+F=\mathrm{I}$, incompatible or disjunctive if $E \wedge F=\mathrm{O}$, and contradictory or orthogonal if $E F=\mathrm{O}$. As in the classical, common sense case, logic contradictory propositions are incompatible. However incompatible propositions are not necessary contradictory as can be easily seen for any two nonorthogonal but not coinciding one-dimensional subspaces. In particular, in quantum logics there exist complementary incompatible pairs $E, F, E \vee F=\mathrm{I}, E \wedge F=\mathrm{O}$ which are not ortho-complementary in the sense $E+F \neq \mathrm{I}$, i.e. $E F \neq \mathrm{O}$ (this would be impossible in the classical case). This is a rigorous logico-mathematical proof of Bohr's complementarity.

As an example, we can consider the proposition that a quantum system is in a stable energy state $E$, and an incompatible proposition $F$, that it collapses at a given time $t$, say. The incompatibility $E \wedge F=\mathrm{O}$ follows from the fact that there is no state in which the system would collapse preserving its energy, however these two propositions are not contradictory (i.e. not orthogonal, $E F \neq \mathrm{O}$ ): the system might not collapse if it is in other than $E$ stationary state (remember the Schrödinger's earlier belief that the energy law is valid only on average, and is violated in the process of radiation). 
In 1952 Wick, Wightman, and Wigner 67 showed that there are physical systems for which not every orthoprojector corresponds to an observable event, so that not every one-dimensional orthoprojector $\rho=P_{\psi}$ corresponding to a wave function $\psi$ is a pure state. This is equivalent to the admission of some selective events which are dispersion-free in all pure states. Jauch and Piron [68] incorporated this situation into quantum logics and proved in the context of this most general approach that the hidden variable interpretation is only possible if the theory is observably wrong, i.e. if incompatible events are always contradictory.

Bell criticized this as well as the Gleason's theorem, but this time his arguments were not based even on the classical ground of usual probability theory. Although he explicitly used the additivity of the probability on the orthogonal events in his counterexample for $\mathbb{H}=\mathbb{C}^{2}$, he questioned : 'That so much follows from such apparently innocent assumptions leads us to question their innocence'. (p.8 in [1]). In fact this was equivalent to questioning the additivity of classical probability on the corresponding disjoint subsets, but he didn't suggest any other complete system of physically reasonable axioms for introducing such peculiar "nonclassical" hidden variables, not even a single counterexample to the orthogonal nonadditivity for the simplest case of quantum bit $\mathbb{H}=\mathfrak{h}$. Thus Bell implicitly rejected classical probability theory in the quantum world, but he didn't want to accept quantum probability as the only possible theory for explaining the microworld.

\subsection{Entanglement and Quantum Causality.}

The nonvalidity of rigorous causality is necessary and not just consistently possible. - Heisenberg.

Thus deterministic causality was questioned by Heisenberg when he analyzed his uncertainty relations. The general consensus among quantum physicists was that there is no positive answer to this question. Max Born even stated:

One does not get an answer to the question, what is the state after collision? but only to the question, how probable is a given effect of the collision?

Einstein was deeply concerned with loss of reality and causality in the treatment of quantum measuring process by Heisenberg and Born. In [70] he suggested a gedanken experiment, now known as EPR paradox. Schrödinger's remained unhappy with Bohr's reply to the EPR paradox, Schrödinger's own analysis was:

It is pretty clear, if reality does not determine the measured value, at least the measurable value determines reality.

In this section we develop this idea of Schrödinger applying it to his explanatory model for the EPR paradox, now is well-know as the Cat of Schrödinger. We shall see how the entanglement, decoherence and the collapse problem can be derived for his cat from purely dynamical arguments of Schrödinger, extending his model to a semi-infinite string of independent cats interacting with a single atom at the boundary of the string by a unitary scattering. We shall see that in such extended system the measurable value (cat is dead or alive) indeed determines reality by simple inference (Bayes conditioning) in the same way as it does in usual classical statistical theory. We shall see that this is possible only due to the Schrödinger's superselection rule which determines his measurable state as reality, i.e. as a classical bit system state with the only two values. This is the only way to keep the 
causality in its weakest, statistical form Later we shall see that quantum causality in the form of a superselection rule is in fact the new postulate of quantum theory which does not contradict to the present formalism and resolves the paradoxes of its statistical interpretation.

2.3.1. Spooky action at distance. After his defeat on uncertainty relations Einstein seemed to have become resigned to the statistical interpretation of quantum theory, and at the 1933 Solvay Congress he listened to Bohr's paper on completeness of quantum theory without objections. In 1935, he launched a brilliant and subtle new attack in a paper 70 with two young co-authors, Podolski and Rosen, which has become of major importance to the world view of physics. They stated the following requirement for a complete theory as a seemingly necessary one:

\section{Every element of physical reality must have a counterpart in the physical theory.}

The question of completeness is thus easily answered as soon as soon as we are able to decide what are the elements of the physical reality. EPR then proposed a sufficient condition for an element of physical reality:

If, without in any way disturbing the system, we can predict with certainty the value of a physical quantity, then there exists an element of physical reality corresponding to this quantity.

Then they designed a thought experiment the essence of which is that two quantum "bits", particle spins of two electrons say, are brought together to interact, and after separation an experiment is made to measure the spin orientation of one of them. The state after interaction is such that the measurement value $v= \pm \frac{1}{2}$ of one particle uniquely determines the spin $z$-orientation $\sigma=\mp \frac{1}{2}$ of the other particle indipendently of its initial state. EPR apply their criterion of local reality: since the value of $\sigma$ can be predicted by measuring $v$ without in any way disturbing $\sigma$, it must correspond to an existing element of physical reality determining the state. Yet the conclusion contradicts a fundamental postulate of quantum mechanics, according to which the sign of spin is not an intrinsic property of a complete description of the spin by state but is evoked only by a process of measurement. Therefore, EPR conclude, quantum mechanics must be incomplete, there must be hidden variables not yet discovered, which determine the spin as an intrinsic property. It seems Einstein was unaware of the von Neumann's hidden variable theorem, although they both had positions at the Institute for Advanced Studies at Princeton (being among the original six mathematics professors appointed there in 1933).

Bohr carefully replied to this challenge by rejecting the assumption of local physical realism as stated by EPR [71]: 'There is no question of a mechanical disturbance of the system under investigation during the last critical stage of the measuring procedure. But even at this stage there is essentially a question of an influence on the very conditions which define the possible types of predictions regarding the future behavior of the system'. This influence became notoriously famous as Bohr's spooky action at a distance. He had obviously meant the semi-classical model of measurement, when one can statistically infer the state of one (quantum) part of a system immediately after observing the other (classical) part, whatever the distance between them. In fact, there is no paradox of "spooky action at distance" in the classical case. The statistical inference, playing the role of such immediate action, is simply based on the Bayesian selection rule of a posterior state from the prior 
mixture of all such states, corresponding to the possible results of the measurement. Bohr always emphasized that one must treat the measuring instrument classically (the measured spin, or another bit interacting with this spin, as a classical bit), although the classical-quantum interaction should be regarded as quantum. The latter follows from non-existence of semi-classical Poisson bracket (i.e. classicalquantum potential interaction) for finite systems. Schrödinger clarified this point more precisely then Bohr, and he followed in fact the mathematical pattern of von Neumann measurement theory.

2.3.2. Releasing Schrödinger's cat. Motivated by EPR paper, in 1935 Schrödinger published a three part essay [72] on 'The Present Situation in Quantum Mechanics'. He turns to EPR paradox and analyses completeness of the description by the wave function for the entangled parts of the system. (The word entangled was introduced by Schrödinger for the description of nonseparable states.) He notes that if one has pure states $\psi(\sigma)$ and $\chi(v)$ for each of two completely separated bodies, one has maximal knowledge, $\psi_{1}(\sigma, v)=\psi(\sigma) \chi(v)$, for two taken together. But the converse is not true for the entangled bodies, described by a non-separable wave function $\psi_{1}(\sigma, v) \neq \psi(\sigma) \chi(v)$ :

Maximal knowledge of a total system does not necessary imply maximal knowledge of all its parts, not even when these are completely separated one from another, and at the time can not influence one another at all.

To make absurdity of the EPR argument even more evident he constructed his famous burlesque example in quite a sardonic style. A cat is shut up in a steel chamber equipped with a camera, with an atomic mechanism in a pure state $\rho_{0}=P_{\psi}$ which triggers the release of a phial of cyanide if an atom disintegrates spontaneously, and this proposition is represented by a one-dimensional projector $F$. It is assumed that it might not disintegrate in a course of an hour $t=1$ with probability $\operatorname{Tr}\left(E P_{\psi}\right)=1 / 2$, where $E=\mathrm{I}-F$. If the cyanide is released, the cat dies, if not, the cat lives. Because the entire system is regarded as quantum and closed, after one hour, without looking into the camera, one can say that the entire system is still in a pure state in which the living and the dead cat are smeared out in equal parts.

Schrödinger resolves this paradox by noting that the cat is a macroscopic object, the states of which (alive or dead) could be distinguished by a macroscopic observation as distinct from each other whether observed or not. He calls this 'the principle of state distinction' for macroscopic objects, which is in fact the postulate that the directly measurable system (consisting of the cat) must be classical:

It is typical in such a case that an uncertainty initially restricted to an atomic domain has become transformed into a macroscopic uncertainty which can be resolved through direct observation.

The dynamical problem of the transformation of the atomic, or "coherent" uncertainty, corresponding to a probability amplitude $\psi(\sigma)$, into a macroscopic uncertainty, corresponding to a mixed state $\rho$, is called quantum decoherence problem. Thus he suggested that the solution of EPR paradox is in non-equivalence of two spins in this thought experiment, one being observed and thus must be open macroscopic subsystem, and the other, nonobserved, can stay microscopic and closed. This was the true reason why he replaced the observed spin by the classical two state cat, and the other by an unstable atom as a quantum model of 
a two level closed system. The only problem was to construct the corresponding classical-quantum transformation in a consistent way.

In order to make this idea clear, let us formulate the dynamical Schrödinger's cat problem in the purely mathematical way. For the notational simplicity instead of the values $\pm 1 / 2$ for the spin-variables $\sigma$ and $v$ we shall use the indexing values $\tau, v \in\{0,1\}$ describing the states of a "bit", the "atomic" system of the classical information theory.

Consider the atomic mechanism as a quantum "bit" with Hilbert space $\mathfrak{h}=\mathbb{C}^{2}$, the pure states of which are described by $\psi$-functions of the variable $\tau \in\{0,1\}$, i.e. by 2 -columns with scalar (complex) entries $\psi(\tau)=\langle\tau| \psi$ defining the probabilities $|\psi(\tau)|^{2}$ of the quantum elementary propositions corresponding to $\tau=0,1$. If atom is disintegrated, $\psi=|1\rangle$ corresponding to $\tau=1$, if not, $\psi=|0\rangle$ corresponding to $\tau=0$. The Schrödinger's cat is a classical bit with only two pure states $v \in\{0,1\}$ which can be identified with the Krönicker delta probability distributions $\delta_{0}(v)$ when alive $(v=0)$ and $\delta_{1}(v)$ when dead $(v=1)$. These pure and even mixed states of the cat can also be described by the complex amplitudes $\chi(v)=\langle v| \chi$ as it were initially quantum bit. However the 2-columns $\chi$ are uniquely defined by the probabilities $|\chi(v)|^{2}$ not just up to a phase constant as in the case of the atom (only constants commute with all atomic observables $\mathrm{B} \in \mathfrak{L}$ on the Hilbert space $\mathfrak{h}$ ), but up to a phase function of $v$ (the phase multiplier of $\chi \in \mathfrak{g}, \mathfrak{g}=\mathbb{C}^{2}$ commuting with all cat observables $c(v)$ ) if the cat is considered as being classical. Initially the cat is alive, so its amplitude defined as $\chi_{0}=|0\rangle$ up to a phase function by the probability distribution $\delta_{0} \equiv \delta$ on $\{0,1\}$, is equal 1 if $v=0$, and 0 if $v=1$ as $\langle v \mid 0\rangle=\delta_{0}(v)$.

The dynamical interaction in this semiclassical system can be described by the unitary transformation

$$
\mathrm{S}=E \otimes \hat{1}+F \otimes \sigma_{1}=\sigma_{1}^{\mathrm{X} \otimes \hat{1}}
$$

in $\mathfrak{h} \otimes \mathfrak{g}$ as it was purely quantum composed system. Here $\sigma_{1}$ is the unitary flipoperator $\sigma_{1} \chi(v)=\chi(v \triangle 1)$ in $\mathfrak{g}$, where $v \Delta \tau=|v-\tau|=\tau \Delta v$ is the difference $(\bmod 2)$ on $\{0,1\}$, and $\mathrm{X}=0 E+1 F$ is the orthoprojector $F$ in $\mathfrak{h}$. This is the only meaningful interaction affecting the cat but not the atom after the hour in a way suggested by Schrödinger,

$$
\mathrm{S}[\psi \otimes \chi](\tau, v):=\langle\tau, v| \mathrm{S}(\psi \otimes \chi)=\psi(\tau) \chi((v \triangle \tau))
$$

where $\langle\tau, v|=\langle\tau| \otimes\langle v|$. Applied to the initial product-state $\psi_{0}=\psi \otimes \delta$ corresponding to $\chi_{0}=\delta$ it has the resulting probability amplitude

$$
\psi_{1}(\tau, v)=\psi(\tau) \delta(v \triangle \tau)=0 \quad \text { if } \quad \tau \neq v
$$

Because the initial state $\delta$ is pure for the cat considered either as classical bit or quantum, the initial composed state $\psi_{0}=\psi \otimes \delta$ is also pure even if this system is considered as semiquantum, corresponding to the Cartesian product $(\psi, 0)$ of the initial pure classical $v=0$ and quantum states $\psi \in \mathfrak{h}$. Despite this fact one can easily see that the unitary operator $S$ induces in $\mathbb{H}=\mathfrak{h} \otimes \mathfrak{g}$ the mixed state for the quantum-classical system, although it is still described by the vector $\psi_{1}=\mathrm{S} \psi_{0} \in \mathbb{H}$ as the wave function $\psi_{1}(\tau, v)$ of the "atom+cat" corresponding to $\psi_{0}=\psi \otimes \delta$.

Indeed, the potential observables of such a system at the time of observation $t=1$ are all operator-functions $X$ of $v$ with values $X(v)$ in Hermitian $2 \times 2$-matrices, represented as block-diagonal $(\tau, v)$-matrices $\hat{X}=\left[X(v) \delta_{v^{\prime}}^{v}\right]$ of the multiplication 
$X(v) \psi_{1}(\cdot, v)$ at each point $v \in\{0,1\}$. This means that the amplitude $\psi_{1}$ (and its density matrix $\left.\omega=P_{\psi_{1}}\right)$ induces the same expectations

$$
\langle\hat{X}\rangle=\sum_{v} \psi_{1}(v)^{\dagger} X(v) \psi_{1}(v)=\sum_{v} \operatorname{Tr} X(v) \varrho(v)=\operatorname{Tr} \hat{X} \hat{\varrho}
$$

as the block-diagonal density matrix $\hat{\varrho}=\left[\varrho(v) \delta_{v^{\prime}}^{v}\right]$ of the multiplication by

$$
\varrho(v)=F(v) P_{\psi} F(v)=\pi(v) P_{F(v) \psi}
$$

where $\pi(v)=|\psi(v)|^{2}, F(v)=P_{\delta_{v}}$ is the projection operator $E$ if $v=0$ and $F$ if $v=1$ :

$$
[F(v) \psi](\tau)=\delta(v \triangle \tau) \psi(\tau)=\psi(v) \delta_{v}(\tau)
$$

and $P_{F(v) \psi}=P_{\delta_{v}}$ is also projector onto $\delta_{v}(\cdot)=\delta(\cdot \triangle v)$. The $4 \times 4$-matrix $\hat{\varrho}$ is a mixture of two orthogonal projectors $P_{\delta_{v}} \otimes P_{\delta_{v}}, v=0,1$ :

$$
\hat{\varrho}=\left[P_{\delta_{v}} \delta_{v^{\prime}}^{v} \pi(v)\right]=\sum_{v=0}^{1} \pi(v) P_{\delta_{v}} \otimes P_{\delta_{v}} .
$$

The only remaining problem is to explain how the cat, initially interacting with atom as a quantum bit described by the algebra $\mathcal{A}=\mathcal{B}(\mathfrak{g})$ of all operators on $\mathfrak{g}$, after the measurement becomes classical, described by the commutative subalgebra $\mathcal{C}=\mathcal{D}(\mathfrak{g})$ of all diagonal operators on $\mathfrak{g}$. As will be shown in the next section even, this can be done in purely dynamical terms if the system "atom plus cat" is extended to an infinite system by adding a quantum string of "incoming cats" and a classical string of "outgoing cats" with a potential interaction (2.16) with the atom at the boundary. The free dynamics in the strings is modeled by the simple shift which replaces the algebra $\mathcal{A}$ of the quantum cat at the boundary by the algebra $\mathcal{C}$ of the classical one, and the total discrete-time dynamics of this extended system is induced on the infinite semi-classical algebra of the "atom plus strings" observables by a unitary dynamics on the extended Hilbert space $\mathcal{H}=\mathbb{H} \otimes \mathcal{H}_{0}$. Here $\mathbb{H}=\mathfrak{h} \otimes \mathfrak{g}$, and $\mathcal{H}_{0}$ is generated by the orthonormal infinite products $\left|\tau_{0}^{\infty}, v_{0}^{\infty}\right\rangle=\otimes\left|\tau_{i}, v_{i}\right\rangle$ for all the strings of quantum $\tau_{0}^{\infty}=\left(\tau_{1}, \tau_{2}, \ldots\right)$ and classical $v_{0}^{\infty}=\left(v_{1}, v_{2}, \ldots\right)$ bits with almost all (but finite number) of $\tau_{n}$ and $v_{m}$ being zero. In this space the total dynamics is described by the single-step unitary transformation

$$
U:\left|\tau_{0}, v\right\rangle \otimes\left|\tau \sqcup \tau_{0}^{\infty}, v_{0}^{\infty}\right\rangle \mapsto\left|\tau_{0}, \tau_{0}+\tau\right\rangle \otimes\left|\tau_{0}^{\infty}, v \sqcup v_{0}^{\infty}\right\rangle,
$$

incorporating the shift and the scattering $\mathrm{S}$, where $\tau+v$ is the sum $\bmod 2$ (which coincides with $\tau \triangle v=|\tau-v|)$, and

$$
\left|\tau_{0}^{\infty}, v \sqcup v_{0}^{\infty}\right\rangle=\left|\tau_{1}, \tau_{2}, \ldots\right\rangle \otimes\left|v, v_{1}, v_{2}, \ldots\right\rangle, \quad \tau, v \in\{0,1\}
$$

are the shifted orthogonal vectors which span the whole infinite Hilbert product space $\mathcal{H}_{0}=\otimes_{r>0} \mathbb{H}_{r}$ of $\mathbb{H}_{r}=\mathfrak{h}_{r} \otimes \mathfrak{g}_{r}$ (the copies of the four-dimensional Hilbert space $\mathbb{H})$. Thus the states $\left|\tau_{0}^{\infty}, v_{0}^{\infty}\right\rangle=\left|\tau_{0}^{\infty}\right\rangle \otimes\left|v_{0}^{\infty}\right\rangle$ can be interpreted as the products of two discrete waves interacting only at the boundary via the atom. The incoming wave $\left|\tau_{0}^{\infty}\right\rangle$ is the quantum probability amplitude wave describing the state of "input quantum cats". The outgoing wave $\left|v_{0}^{\infty}\right\rangle$ is the classical probability amplitude wave describing the states of "output classical cats". 
2.3.3. The measurement problem. Inspired by Bohr's complementarity principle, von Neumann proposed even earlier the idea that every quantum measuring process involves an unanalysable element. He postulated [63] that, in addition to the continuous causal propagation $\psi(0) \mapsto \psi(t)$ of the wave function generated by the Schrödinger equation, the function $\psi$ undergoes a discontinuous, irreversible instantaneous change due to an action of the observer on the object preparing the measurement at the time $t$. Just prior to the reading of measurement result of an event $F$, disintegration of the atom, say, the quantum pure state $\sigma=P_{\psi}$ changes to the mixed one

$$
\rho=\lambda P_{E \psi}+\mu P_{F \psi}=E \sigma E+F \sigma F,
$$

where $E=I-F$ is the orthocomplement event, and

$$
\lambda=\|E \psi\|^{2}=\operatorname{Tr} E \rho, \quad \mu=\|F \psi\|^{2}=\operatorname{Tr} F \rho .
$$

are the probabilities of $E$ and $F$. Such change is projective as shown in the second part of this equation, and it is called the von Neumann projection postulate.

This linear irreversible decoherence process should be completed by the nonlinear, acausal random jump to one of the pure states

$$
\rho \mapsto P_{E \psi}, \text { or } \rho \mapsto P_{F \psi}
$$

depending on whether the tested event $F$ is false (the cat is alive, $\psi_{0}=\lambda^{-1 / 2} E \psi$ ), or true (the cat is dead, $\psi_{1}=\mu^{-1 / 2} F \psi$ ). This final step is the posterior prediction, called filtering of the decoherent mixture of $\psi_{0}$ and $\psi_{1}$ by selection of only one result of the measurement, and is an unavoidable element in every measurement process relating the state of the pointer of the measurement (in this case the cat) to the state of the whole system. This assures that the same result would be obtained in case of immediate subsequent measurement of the same event $F$. The resulting change of the prior wave-function $\psi$ is described up to normalization by one of the projections

$$
\psi \mapsto E \psi, \quad \psi \mapsto F \psi
$$

and is sometimes called the Lüders projection postulate [73].

Although unobjectionable from the purely logical point of view the von Neumann theory of measurement soon became the target of severe criticisms. Firstly it seams radically subjective, postulating the spooky action at distance in a purely quantum system instead of deriving it. Secondly the von Neumann analysis is applicable to only the idealized situation of discrete instantaneous measurements.

The first objection can be regarded as a result of misinterpretation of the projection postulate $\sigma \mapsto \rho$ which can be avoided by its more adequate formulation in the "Heisenberg picture" for any atomic observable $\mathrm{B}$ as the transformation $\mathrm{B} \mapsto \mathrm{A}$,

$$
\mathrm{A}=E \mathrm{~B} E+F \mathrm{~B} F, \quad \mathrm{~B} \in \mathcal{B}(\mathfrak{h}),
$$

where $\mathcal{B}(\mathfrak{h})$ is the algebra of operators on $\mathfrak{h}$. Note that this transformation is irreversible, so the "Heisenberg" and "Schrödinger" pictures for measurements are no longer unitary equivalent as the matter of choice of the mathematically equivalent representations as it is in the case of the conservative (reversible) dynamical transformations. However these two pictures are physically (statistically) equivalent as they give the same prediction of the expectations just prior the measurement:

$$
\operatorname{Tr} \mathrm{B} \sigma=\langle\psi|E \mathrm{~A} E+F \mathrm{~A} F| \psi\rangle=\operatorname{Tr} \mathrm{A} \rho .
$$


Indeed, thus reformulated, the projection postulate can be interpreted as the reduction of the set of all potential observables to only those compatible with the measurement in the result of the preparation of this measurement. There is nothing subjective in this, the states $\sigma=P_{\psi}$ describing the reality by wave functions $\psi$ prior the measurement are not changed, and the reduction of potential observables is merely a rule to satisfy the Bohrs complementarity for the given measurement. However after the reduction of quantum potentialities to only those which are compatible with the measurement, the pure state $\sigma$ becomes mixed even without the change of $\psi$, and this state can be described not only by the prior density operator $P_{\psi}$ but by the mixture $\rho$ in (2.21) of the posterior $P_{E \psi}$ and $P_{F \psi}$ such that $\operatorname{Tr} A P_{\psi}=\operatorname{Tr} \mathrm{A} \rho$ for all reduced observables A.

As we already mentioned when discussing the EPR paradox, the process of filtering $\rho \mapsto P_{F(v) \psi}$ is free from any conceptual difficulty if it is understood as the statistical inference about a mixed state in an extended stochastic representation of the quantum system as a part of a semiclassical one, based upon the results $v$ of observation in its classical part. As we shall see in the next section this is as simple as the transition from the prior to posterior classical probabilities by the conditioning upon the results of statistical inference. Note that in classical statistics due to complete commutativity such conditioning is always possible, and this is why there is no measurements problem in classical physics.

In the previous section we mentioned that the amount of commutativity which is necessary to derive the projection postulate as the result of inference, can be dynamically achieved by extending the system to the infinity. In the next section we shall show how to do this in the general case, but here let us demonstrate this for the dynamical model of "cat", identifying the quantum system in question with the Schrödinger's atom. The event $E$ (the atom exists) will correspond then to $v=0$ (the cat is alive), $E=F(0)$, and the complementary event will be $F=F(1)$.

Consider the semi-classical string of "incoming and outgoing cats" as the quantum and classical bits moving freely in the opposite directions along the discrete coordinate $r \in \mathbb{N}$. The Hamiltonian interaction of the quantum (incoming) cats with the atom at the boundary $r=0$ is described by the unitary scattering (2.16). The whole system is described by the unitary transformation 2.20 which induces an injective endomorphism $\vartheta(A)=U^{\dagger} A U$ on the infinite product algebra $\mathfrak{A}=\mathcal{A} \otimes \mathfrak{A}_{0}$ of the atom-cat observables $\hat{X} \in \mathcal{A}$ at $r=0$ and other quantum-classical cats $\mathfrak{A}_{0}=\otimes_{r>0} \mathcal{A}_{r}$. Here $\mathcal{A}=\mathcal{B}(\mathfrak{h}) \otimes \mathcal{C}$ is the block-diagonal algebra of operator-valued functions $\{0,1\} \ni v \mapsto X(v)$ describing the observables of the string boundary on the Hilbert space $\mathfrak{h} \otimes \mathfrak{g}$, where $\mathfrak{h}=\mathbb{C}^{2}=\mathfrak{g}$, and $\mathcal{A}_{r}=\mathcal{B}\left(\mathfrak{h}_{r}\right) \otimes \mathcal{C}_{r}$ are copies of $\mathcal{A}_{0}$ represented on tensor products $\mathfrak{h}_{r} \otimes \mathfrak{g}_{r}$ of the copies $\mathfrak{h}_{r}=\mathbb{C}^{2}=\mathfrak{g}_{r}$ at $r>0$.

The input quantum probability waves $\left|\tau_{0}^{\infty}\right\rangle=\otimes_{r>0}\left|\tau_{r}\right\rangle$ describe initially disentangled pure states on the noncommutative algebra $\mathcal{B}\left(\mathcal{H}_{0}\right)=\otimes_{r>0} \mathcal{B}\left(\mathfrak{h}_{r}\right)$ of "incoming quantum cats" in $\mathcal{H}_{0}=\otimes_{r>0} \mathfrak{h}_{r}$, and the output classical probability waves $\left|v_{0}^{\infty}\right\rangle=\otimes_{r>0}\left|v_{r}\right\rangle$ describe initially pure states on the commutative algebra $\mathfrak{C}_{0}=\otimes_{r>0} \mathcal{C}_{r}$ of "outgoing classical cats" in $\mathcal{G}_{0}=\otimes_{r>0} \mathfrak{g}_{r}$. At the boundary $r=0$ there is a transmission of information from the quantum algebra $\mathcal{B}(\mathcal{H})$ on $\mathcal{H}=\mathfrak{h} \otimes \mathcal{H}_{0}$ to the classical one $\mathfrak{C}=\mathcal{C} \otimes \mathfrak{C}_{0}$ on $\mathcal{G}=\mathfrak{g} \otimes \mathcal{G}_{0}$ which is induced by the Heisenberg transformation $\vartheta: \mathfrak{A} \mapsto \mathfrak{A}$. Note that although the Schrödinger transformation $U$ is reversible on $\mathbb{H}=\mathcal{H} \otimes \mathcal{G}, U^{-1}=U^{\dagger}$, and thus the Heisenberg endomorphism $\vartheta$ is one-to-one on the semi-commutative algebra $\mathfrak{A}=\mathcal{B}(\mathcal{H}) \otimes \mathfrak{C}$, it describes an irreversible dynamics because the image subalgebra $\vartheta(\mathfrak{A})=U^{\dagger} \mathfrak{A} U$ 
of the algebra $\mathfrak{A}$ does not coincide with $\mathfrak{A} \subset \mathcal{B}(\mathbb{H})$. The initially distinguishable pure states on $\mathfrak{A}$ may become identical and mixed on the smaller algebra $U^{\dagger} \mathfrak{A} U$, and this explains the decoherence.

Thus, this dynamical model explains that the origin of the von Neumann irreversible decoherence $\sigma=P_{\psi} \mapsto \rho$ of the atomic state is in the ignorance of the result of the measurement described by the partial tracing over the cat's Hilbert space $\mathfrak{g}=\mathbb{C}^{2}$ :

$$
\rho=\operatorname{Tr}_{\mathfrak{g}} \hat{\varrho}=\sum_{v=0}^{1} \pi(v) P_{\delta_{v}}=\varrho(0)+\varrho(1)
$$

where $\varrho(v)=|\psi(v)|^{2} P_{\delta_{v}}$. It has entropy $\mathrm{S}(\rho)=-\operatorname{Tr} \rho \log \rho$ of the compound state $\hat{\varrho}$ of the combined semi-classical system prepared for the indirect measurement of the disintegration of atom by means of cat's death:

$$
\mathbf{S}(\rho)=-\sum_{v=0}^{1}|\psi(v)|^{2} \log |\psi(v)|^{2}=\mathbf{S}(\hat{\varrho})
$$

It is the initial coherent uncertainty in the pure quantum state of the atom described by the wave-function $\psi$ which is equal to one bit in the case $|\psi(0)|^{2}=1 / 2=|\psi(1)|^{2}$. Each step of the unitary dynamics adds this entropy to the total entropy of the state on $\mathfrak{A}$ at the time $t \in \mathbb{N}$, so the total entropy produced by this dynamical decoherence model is equal exactly $t$.

The described dynamical model of the measurement interprets filtering $\rho \mapsto \sigma_{v}$ simply as the conditioning

$$
\sigma_{v}=\varrho(v) / \pi(v)=P_{\delta_{v}}
$$

of the joint classical-quantum state $\varrho(\cdot)$ with respect to the events $F(v)$ by the Bayes formula which is applicable due to the commutativity of actually measured observables $C \in \mathcal{C}$ (the life observables of cat at the time $t=1$ ) with any other potential observable of the combined semi-classical system.

Thus the atomic decoherence is derived from the unitary interaction of the quantum atom with the cat which should be treated as classical due to the projection superselection rule in the "Heisenberg" picture of von Neumann measurement. The spooky action at distance, affecting the atomic state by measuring $v$, is simply the result of the statistical inference (prediction after the measurement) of the atomic posterior state $\sigma_{v}=P_{\delta_{v}}$ : the atom disintegrates if and only if the cat is dead.

A formal derivation of the von-Neumann-Lüders projection postulate and the decoherence in the case of more general (discrete and continuous) spectra by explicit construction of unitary transformation in the extended semi-classical system as outlined in [2, 75] is given in the next section. An extension of this analysis to quantum continuous time and spectra will also be considered in the last sections.

\section{The Dynamical Solution for Quantum Measurement}

How wonderful we have met with a paradox, now we have some hope of making progress - Niels Bohr. 


\subsection{Instantaneous Quantum Measurements.}

If we have to go on with these dammed quantum jumps, then I'm sorry that I ever got involved - Schrödinger.

In this Chapter we present the main ideas of modern quantum measurement theory and the author's views on the quantum measurement problem which might not coincide with the present scientific consensus that this problem is unsolvable in the standard framework, or at least unsolved [74]. It will be shown that there exists such solution along the line suggested by the great founders of quantum theory Schrödinger, Heisenberg and Bohr. We shall see that von Neumann only partially solved this problem which he studied in his Mathematical Foundation of Quantum Theory [63], and that the direction in which the solution might be found was envisaged by the modern quantum philosopher J Bell [1].

Here we develope the approach suggested in the previous section for solving the famous Schrödinger's cat paradox. We shall see that even the most general quantum decoherence and wave packet reduction problem for an instantaneous or even sequential measurements can be solved in a canonical way which corresponds to adding a single initial cat's state. This resolves also the other paradoxes of quantum measurement theory in a constructive way, giving exact nontrivial models for the statistical analysis of quantum observation processes determining the reality underlying these paradoxes. Conceptually it is based upon a new idea of quantum causality as a superselection rule called the Nondemolition Principle [2] which divides the world into the classical past, forming the consistent histories, and the quantum future, the state of which is predictable for each such history. This new postulate of quantum theory making the solution of quantum measurement possible can not be contradicted by any experiment as we prove that any sequence of usual, "demolition" measurements based on the projection postulate or any other phenomenological measurement theories is statistically equivalent, and in fact can be dynamically realized as a simultaneous nondemolition measurement in a canonically extended infinite semi-quantum system. The nondemolition models give exactly the same predictions as the orthodox, "demolition" theories, but they do not require the projection or any other postulate except the causality (nondemolition) principle.

3.1.1. Generalized reduction and its dilation. Von Neumann's projection postulate, even reformulated in Heisenberg picture, is only a phenomenological reduction principle which requires a dynamical justification. Before formulating this quantum measurement problem in the most interesting time-continuous case, let us consider how the reduction principle can be generalized to include not only discrete but also continuous measurement spectra for a single time $t$.

The generalized reduction of the wave function $\psi(x)$, corresponding to a complete measurement with discrete or even continuous data $y$, is described by a function $V(y)$ whose values are linear operators $\mathfrak{h} \ni \psi \mapsto V(y) \psi$ for each $y$ which are not assumed to be isometric on the quantum system Hilbert space $\mathfrak{h}$, $V(y)^{\dagger} V(y) \neq I$, but have the following normalization condition. The resulting wave-function

$$
\psi_{1}(x, y)=[V(y) \psi](x)
$$


is normalized with respect to a given measure $\mu$ on $y$ in the sense

$$
\iint|[V(y) \psi](x)|^{2} \mathrm{~d} \lambda_{x} \mathrm{~d} \mu_{y}=\int|\psi(x)|^{2} \mathrm{~d} \lambda_{x}
$$

for any probability amplitude $\psi$ normalized with respect to a measure $\lambda$ on $x$. This can be written as the isometry condition $\mathrm{V}^{\dagger} \mathrm{V}=\mathrm{I}$ of the operator $\mathrm{V}: \psi \mapsto V(\cdot) \psi$ in terms of the integral

$$
\int_{y} V(y)^{\dagger} V(y) \mathrm{d} \mu_{y}=\mathrm{I}, \quad \text { or } \quad \sum_{y} V(y)^{\dagger} V(y)=\mathrm{I} .
$$

with respect to the base measure $\mu$ which is usually the counting measure, $\mathrm{d} \mu_{y}=1$ in the discrete case, e.g. in the case of two-point variables $y=v$ (EPR paradox, or Schrödinger cat with the projection-valued $V(v)=F(v))$. The general case of orthoprojectors $V(y)=F(y)$ corresponds to the Krönicker $\delta$-function $V(y)=\delta_{y}^{\mathrm{X}}$ of a self-adjoint operator $\mathrm{X}$ on $\mathfrak{h}$ with the discrete spectrum coinciding with the measured values $y$.

As in the simple example of the Schrödinger's cat, the unitary realization of such $V$ can always be constructed in terms of a unitary transformation on an extended Hilbert space $\mathfrak{h} \otimes \mathfrak{g}$ and a normalized wave function $\chi^{\circ} \in \mathfrak{g}$. It is easy to find such unitary dilation of any reduction family $V$ of the form

$$
V(y)=\mathrm{e}^{-i \mathrm{E} / \hbar} \exp \left[-\mathrm{X} \frac{\mathrm{d}}{\mathrm{d} y}\right] \varphi(y)=\mathrm{e}^{-i \mathrm{E} / \hbar} F(y),
$$

given by a normalized wave-function $\varphi \in L^{2}(\mathbb{G})$ on a cyclic group $\mathbb{G} \ni y$ (e.g. $\mathbb{G}=\mathbb{R}$ or $\mathbb{G}=\mathbb{Z})$. Here the shift $F(y)=\varphi(y-\mathrm{X})$ of $\chi^{\circ}=\varphi$ by a measured operator $\mathrm{X}$ in $\mathfrak{h}$ is well-defined by the unitary shifts $\exp \left[-x \frac{\mathrm{d}}{\mathrm{d} y}\right]$ in $\mathfrak{g}=L^{2}(\mathbb{G})$ in the eigen-representation of any selfadjoint $\mathrm{X}$ having the spectral values $x \in \mathbb{G}$, and $\mathrm{E}=\mathrm{E}^{\dagger}$ is any free evolution action after the measurement. As was noted by von Neumann for the case $\mathbb{G}=\mathbb{R}$ in $\left[63\right.$, the operator $S=\exp \left[-\mathrm{X} \frac{\mathrm{d}}{\mathrm{d} y}\right]$ is unitary in $\mathfrak{h} \otimes \mathfrak{g}$, and it coincides on $\psi \otimes \varphi$ with the isometry $\mathrm{F}=\mathrm{S}(\mathrm{I} \otimes \varphi)$ on each $\psi \in \mathfrak{h}$ such that the unitary operator $\mathrm{W}=\mathrm{e}^{-i \mathrm{E} / \hbar} \mathrm{S}$ dilates the isometry $\mathrm{V}=\mathrm{e}^{-i \mathrm{E} / \hbar} \mathrm{F}$ in the sense

$$
\mathrm{W}\left(\psi \otimes \chi^{\circ}\right)=\mathrm{e}^{-i \mathrm{E} / \hbar} \mathrm{S}(\psi \otimes \varphi)=\mathrm{e}^{-i \mathrm{E} / \hbar} \mathrm{F} \psi, \quad \forall \psi \in \mathfrak{h} .
$$

The wave function $\chi^{\circ}=\varphi$ defines the initial probability distribution $|\varphi(y)|^{2}$ of the pointer coordinate $y$ which can be dispersionless only if $\varphi$ is an eigen-function of the pointer operator $Y=\hat{y}$ (multiplication operator by $y$ in $\mathfrak{g}$ ) corresponding to a discrete spectral value $y^{\circ}$ as a predetermined initial value of the pointer, $y^{\circ}=0$ say. This corresponds to ortho-projectors $V(y)=\delta_{y}^{\mathrm{X}}=F(y)(\mathrm{E}=\mathrm{O})$ indexed by $y$ from a discrete cycle group, $y \in \mathbb{Z}$ for the discrete $\mathrm{X}$ having eigenvalues $x \in \mathbb{Z}$ say. Thus the projection postulate is always dilated by such shift operator $\mathrm{S}$ with $\chi^{\circ}(y)=\delta_{y}^{0}$ given as the eigen-function $\varphi(y)=\delta_{y}^{0}$ corresponding to the initial value $y=0$ for the pointer operator $Y=\hat{y}$ in $\mathfrak{g}=L^{2}(\mathbb{Z})$ (In the case of the Schrödinger's cat $U$ was simply the shift $W(\bmod 2)$ in $\left.\mathfrak{g}=L^{2}(0,1):=\mathbb{C}^{2}\right)$.

There exist another, canonical construction of the unitary operator $\mathrm{W}$ with the eigen-vector $\chi^{\circ} \in \mathfrak{g}$ for a 'pointer observable' $Y$ in an extended Hilbert space $\mathfrak{g}$ even if $y$ is a continuous variable of the general family $V(y)$. More precisely, it can always be represented on the tensor product of the system space $\mathfrak{h}$ and the space 
$\mathfrak{g}=\mathbb{C} \oplus L_{\mu}^{2}$ of square-integrable functions $\chi(y)$ defining also the values $\chi\left(y^{\circ}\right) \in \mathbb{C}$ at an additional point $y^{\circ} \neq y$ corresponding to the absence of a result $y$ and $\chi^{\circ}=1 \oplus 0$ such that

$$
\langle x| V(y) \psi=(\langle x| \otimes\langle y|) \mathrm{W}\left(\psi \otimes \chi^{\circ}\right), \quad \forall \psi \in \mathfrak{h}
$$

for each measured value $y \neq y^{\circ}$.

Now we prove this unitary dilation theorem for the general $V(y)$ by the explicit construction of the matrix elements $\mathrm{W}_{y^{\prime}}^{y}$ in the unitary block-operator $\mathrm{W}=\left[\mathrm{W}_{y^{\prime}}^{y}\right]$ defined as $(\mathrm{I} \otimes\langle y|) \mathrm{W}\left(\mathrm{I} \otimes\left|y^{\prime}\right\rangle\right)$ by

$$
\psi^{\dagger} \mathrm{W}_{y^{\prime}}^{y} \psi^{\prime}=\left(\psi^{\dagger} \otimes\langle y|\right) \mathrm{W}\left(\psi^{\prime} \otimes\left|y^{\prime}\right\rangle\right),
$$

identifying $y^{\circ}$ with 0 (assuming that $y \neq 0$, e.g. $y=1, \ldots, n$ ). We shall use the short notation $\mathfrak{f}=L_{\mu}^{2}$ for the functional Hilbert space on the measured values $y$ and $\chi^{\circ}=\left|y^{\circ}\right\rangle\left(=|0\rangle\right.$ if $\left.y^{\circ}=0\right)$ for the additional state-vector $\chi^{\circ} \in \mathfrak{g}$, identifying the extended Holbert space $\mathfrak{g}=\mathbb{C} \oplus \mathfrak{f}$ with the space $L_{\mu \oplus 1}^{2}$ of square-integrable functions of all $y$ by the extention $\mu \oplus 1$ of the measure $\mu$ at $y^{\circ}$ as $\mathrm{d} \mu_{y^{\circ}}=1$.

Indeed, we can always assume that $V(y)=\mathrm{e}^{-i \mathrm{E} / \hbar} F(y)$ where the family $F$ is viewed as an isometry $\mathrm{F}: \mathfrak{h} \rightarrow \mathfrak{h} \otimes \mathfrak{f}$ corresponding $\mathrm{F}^{\dagger} \mathrm{F}=\mathrm{I}$ (not necessarily of the form $F(y)=\chi^{\circ}(y-\mathrm{X})$ as in (3.2)). Denoting $\mathrm{e}^{-i \mathrm{E} / \hbar} \mathrm{F}$ as the column of $\mathrm{W}_{0}^{y}, y \neq 0$, and $\mathrm{e}^{-i \mathrm{E} / \hbar} \mathrm{F}^{\dagger}$ as the raw of $\mathrm{W}_{y}^{0}, y \neq 0$, we can compose the unitary block-matrix

$$
\left[\mathrm{W}_{y^{\prime}}^{y}\right]:=\mathrm{e}^{-i \mathrm{E} / \hbar}\left[\begin{array}{rr}
\mathrm{O} & \mathrm{F}^{\dagger} \\
\mathrm{F} & \mathrm{I} \otimes \hat{1}-\mathrm{FF}^{\dagger}
\end{array}\right], \quad \mathrm{I} \otimes \hat{1}=\left[\mathrm{I} \delta_{y^{\prime}}^{y}\right]_{y^{\prime} \neq 0}^{y \neq 0}
$$

describing an operator $\mathrm{W}=\left[\mathrm{W}_{y^{\prime}}^{y}\right]$ on the product $\mathfrak{h} \otimes \mathfrak{g}$, where $\mathfrak{g}=\mathbb{C} \oplus \mathfrak{f}$, represented $\operatorname{ash} \oplus(\mathfrak{h} \otimes \mathfrak{f}), \mathfrak{f}=L_{\mu}^{2}$. It has the adjoint $\mathrm{W}^{\dagger}=\mathrm{e}^{i \mathrm{E} / \hbar} \mathrm{We}^{-i \mathrm{E} / \hbar}$, and obviously

$$
(\mathrm{I} \otimes\langle y|) \mathrm{W}(\mathrm{I} \otimes|0\rangle)=V(y), \quad \forall y \neq 0 .
$$

The unitarity $\mathrm{W}^{-1}=\mathrm{W}^{\dagger}$ of the constructed operator $\mathrm{W}$ is the consequence of the isometricity $\mathrm{F}^{\dagger} \mathrm{F}=\mathrm{I}$ and thus the projectivity $\left(\mathrm{FF}^{\dagger}\right)^{2}=\mathrm{FF}^{\dagger}$ of $\mathrm{FF}^{\dagger}$ and of $\mathrm{I} \otimes \hat{1}-\mathrm{FF}^{\dagger}:$

$$
\mathrm{W}^{\dagger} \mathrm{W}=\left[\begin{array}{ll}
\mathrm{F}^{\dagger} \mathrm{F} & \mathrm{F}^{\dagger}\left(\mathrm{I} \otimes \hat{1}-\mathrm{FF}^{\dagger}\right) \\
\left(\mathrm{I} \otimes \hat{1}-\mathrm{FF}^{\dagger}\right) \mathrm{F} & \mathrm{FF}^{\dagger}+\mathrm{I} \otimes \hat{1}-\mathrm{FF}^{\dagger}
\end{array}\right]=\left[\begin{array}{ll}
\mathrm{I} & \mathrm{O} \\
\mathrm{O} & \mathrm{I} \otimes \hat{1}
\end{array}\right] .
$$

In general the observation may be incomplete: the data $y$ may be the only observable part of a pair $(z, y)$ defining the stochastic wave propagator $V(z, y)$. Consider for simplicity a discrete $z$ such that

$$
V^{\dagger} V:=\sum_{z} \int V(z, y)^{\dagger} V(z . y) \mathrm{d} \mu_{y}=\mathrm{I} .
$$

Then the linear unital map on the algebra $\mathcal{B}(\mathfrak{h}) \otimes \mathcal{C}$ of the completely positive form

$$
\pi(\hat{g} \mathrm{~B})=\sum_{z} \int g(y) V(z, y)^{\dagger} \mathrm{B} V(z . y) \mathrm{d} \mu_{y} \equiv \mathrm{M}[g \pi(\mathrm{B})]
$$

describes the "Heisenberg picture" for generalized von Neumann reduction with an incomplete measurement results $y$. Here $\mathrm{B} \in \mathcal{B}(\mathfrak{h}), \hat{g}$ is the multiplication operator 
by a measurable function of $y$ defining any system-pointer observable by linear combinations of $B(y)=g(y) \mathrm{B}$, and

$$
\pi(y, \mathrm{~B})=\sum_{z} V(z, y)^{\dagger} \mathrm{B} V(z, y), \quad \mathrm{M}[B(y)]=\int B(y) \mathrm{d} \mu_{y} .
$$

The function $y \mapsto \pi(y)$ with values in the completely positive maps $\mathrm{B} \mapsto \pi(y, \mathrm{~B})$, or operations, is the basic tool in the operational approach to quantum measurements. Its adjoint

$$
\pi^{*}(\sigma)=\sum_{z} V(y, z) \sigma V(y, z)^{\dagger} \mathrm{d} \mu_{y}=\pi^{*}(y, \sigma) \mathrm{d} \mu_{y}
$$

is given by the density matrix transformation and it is called the instrument in the phenomenological measurement theories. The operational approach was introduced by Ludwig 80, and the mathematical implementation of the notion of instrument was originated by Davies and Lewis 81.

An abstract instrument now is defined as the adjoint to a unital completely positive map $\pi$ for which $\pi_{y}^{*}(\sigma)$ is a trace-class operator for each $y$, normalized to a density operator $\rho=\int \mathrm{d} \pi_{y}^{*}(\sigma)$. The quantum mixed state described by the operator $\rho$ is called the prior state, i.e. the state which has been prepared for the measurement. A unitary dilation of the generalized reduction (or "instrumental") map $\pi$ was constructed by Ozawa 82], but as we shall now see, this, as well as the canonical dilation (3.4), is only a preliminary step towards the its quantum stochastic realization allowing the dynamical derivation of the reduction postulate as a result of the statistical inference as it was suggested in [2].

3.1.2. The future-past boundary value problem. The additional system of the constructed unitary dilation for the measurement propagator $V(y)$ represents only the pointer coordinate of the measurement apparatus $y$ with the initial value $y=y^{\circ}$ $\left(=0\right.$ corresponding to $\left.\chi^{\circ}=|0\rangle\right)$. It should be regarded as a classical system (like the Schrödinger's cat) at the instants of measurement $t>0$ in order to avoid the applying of the projection postulate for inferences in the auxiliary system. Indeed, the actual events of the measurement can be only those propositions $E$ in the extended system which may serve as the conditions for any other proposition $F$ as a potential in future event, otherwise there can't be any causality even in the weak, statistical sense. This means that future states should be statistically predictable in any prior state of the system in the result of testing the measurable event $E$ by the usual conditional probability (Bayes) formula

$$
\operatorname{Pr}\{F=1 \mid E=1\}=\operatorname{Pr}\{E \wedge F=1\} / \operatorname{Pr}\{E=1\} \quad \forall F,
$$

and this predictability, or statistical causality means that the prior quantum probability $\operatorname{Pr}\{F\} \equiv \operatorname{Pr}\{F=1\}$ must coincide with the statistical expectation of $F$ as the weighted sum

$$
\operatorname{Pr}\{F \mid E\} \operatorname{Pr}\{E\}+\operatorname{Pr}\left\{F \mid E^{\perp}\right\} \operatorname{Pr}\left\{E^{\perp}\right\}=\operatorname{Pr}\{F\}
$$

of this $\operatorname{Pr}\{F \mid E\} \equiv \operatorname{Pr}\{F=1 \mid E=1\}$ and the complementary conditional probability $\operatorname{Pr}\left\{F \mid E^{\perp}\right\}=\operatorname{Pr}\{F=1 \mid E=0\}$. As one can easily see, this is possible if and only if (2.14) holds, i.e. any other future event-orthoprojector $F$ of the extended system must be compatible with the actual event-orthoprojector $E$.

The actual events in the measurement model obtained by the unitary dilation are only the orthoprojectors $E=\mathrm{I} \otimes \hat{1}_{\Delta}$ on $\mathfrak{h} \otimes \mathfrak{g}$ corresponding to the propositions 
" $y \in \Delta$ " where $\hat{1}_{\Delta}$ is the multiplication by the indicator $1_{\Delta}$ for a measurable on the pointer scale subset $\Delta$. Other orthoprojectors which are not compatible with these orthoprojectors, are simply not admissible as the questions by the choice of time arrow. This choice restores the quantum causality as statistical predictability, i.e. the statistical inference made upon the sample data. And the actual observables in question are only the measurable functions $g(y)$ of $y \neq y^{\circ}$ represented on $\mathfrak{f}=$ $L^{2}(\mu)$ by the commuting operators $\hat{g}$ of multiplication by these functions, $\langle y| \hat{g} \chi=$ $g(y) \chi(y)$. As follows from $\mathrm{W}_{0}^{0}=\mathrm{O}$, the initial value $y^{\circ}=0$ is never observed at the time $t=1$ :

$$
\left\|\psi_{1}(0)\right\|^{2}=\|(\mathrm{I} \otimes\langle 0|) \mathrm{W}(\psi \otimes|0\rangle)\|^{2}=\left\|\mathrm{W}_{0}^{0} \psi\right\|^{2}=0, \quad \forall \psi \in \mathfrak{h}
$$

(that is a measurable value $y \neq y^{\circ}$ is certainly observed at $t=1$ ). These are the only appropriate candidates for Bell's "beables", [1], p.174. Indeed, such commuting observables, extended to the quantum counterpart as $\mathrm{G}_{0}=\mathrm{I} \otimes \hat{g}$ on $\mathfrak{h} \otimes \mathfrak{f}$, are compatible with any admissible question or observable $B$ on $\mathfrak{h}$ represented with respect to the output states $\psi_{1}=\mathrm{W} \psi_{0}$ at the time of measurement $t=1$ by an operator $B_{1}=B \otimes \hat{1}$ on $\mathfrak{h} \otimes \mathfrak{f}$. The probabilities (or, it is better to say, the propensities) of all such questions are the same in all states whether an observable $\mathrm{G}_{0}$ was measured but the result not read, or it was not measured at all. In this sense the measurement of $\mathrm{G}_{0}$ is called nondemolition with respect to the system observables $\mathrm{B}_{1}$, they do not demolish the propensities, or prior expectations of $\mathrm{B}$. However as we shall show now they are not necessary compatible with the same operators $\mathrm{B}$ of the quantum system at the initial stage and currently represented as $\mathrm{WB}_{0} \mathrm{~W}^{\dagger}$ on $\psi_{1}$, where $\mathrm{B}_{0}=\mathrm{B} \otimes \hat{1}$ is the Schrödinger representation of $\mathrm{B}$ at the time $t=0$ on the corresponding input states $\psi_{0}=\mathrm{W}^{\dagger} \psi_{1}$ in $\mathfrak{h} \otimes \mathfrak{g}$.

Indeed, we can see this on the example of the Schrödinger cat, where $\mathrm{W}$ is the flip $S$ in $\mathfrak{g}=\mathbb{C}^{2}(\operatorname{shift} \bmod 2)$. In this case the operators the operators $\hat{g}_{1}$ in the Heisenberg picture $\mathrm{G}=\mathrm{S}^{\dagger} \mathrm{G}_{0} \mathrm{~S}$ are represented on $\mathfrak{h} \otimes \mathfrak{g}$ as the diagonal operators $\mathrm{G}=\left[g(\tau+v) \delta_{\tau^{\prime}}^{\tau} \delta_{v^{\prime}}^{v}\right]$ of multiplication by $g(\tau+v)$, where the sum $\tau+v=|\tau-v|$ is modulo 2. Obviously they do not commute with $\mathrm{B}_{0}$ unless $\mathrm{B}$ is also a diagonal operator $\hat{f}$ of multiplication by a function $f(\tau)$, in which case

$$
\left[\mathrm{B}_{0}, \mathrm{G}\right] \psi_{0}(\tau, v)=[f(\tau), g(\tau+v)] \psi_{0}(\tau, v)=0, \quad \forall \psi_{0} \in \mathfrak{h} \otimes \mathfrak{g} .
$$

The restriction of the possibilities in a quantum system to only the diagonal operators $\mathrm{B}=\hat{f}$ of the atom which would eliminate the time arrow in the nondemolition condition, amounts to the redundancy of the quantum consideration: all such (possible and actual) observables can be simultaneously represented as classical observables by the measurable functions of $(\tau, v)$.

Thus the constructed semiclassical algebra $\mathcal{B}_{-}=\mathcal{B}(\mathfrak{h}) \otimes \mathcal{C}$ of the Schrödinger's atom and the pointer (dead or alive cat) is not dynamically invariant in the sense that transformed algebra $\mathrm{W}^{\dagger} \mathcal{B}_{-} \mathrm{W}$ does not coincide and is not a part of $\mathcal{B}_{-}$but of $\mathcal{B}_{+}=\mathcal{B}(\mathfrak{h}) \otimes \mathcal{B}(\mathfrak{g})$. This is also true in the general case, unless all the system-pointer observables in the Heisenberg picture are still decomposable,

$$
\mathrm{W}^{\dagger}(\mathrm{B} \otimes \hat{g}) \mathrm{W}=\int|y\rangle g(y) B(y)\langle y| \mathrm{d} \mu_{y},
$$

which would imply $\mathrm{W}^{\dagger} \mathcal{B} \mathrm{W} \subseteq \mathcal{B}$. (Such dynamical invariance of the decomposable algebra, given by the operator-valued functions $B(y)$, can be achieved by this unitary dilations only in trivial cases.) This is why the von Neumann type dilation 
(2.16), and even more general dilations (3.4), or 82, 2] cannot yet be considered as the dynamical solution of the instantaneous quantum measurement problem which we formulate in the following way.

Given a reduction postulate defined by an isometry $V$ on $\mathfrak{h}$ into $\mathfrak{h} \otimes \mathfrak{g}$, find a triple $\left(\mathcal{G}, \mathfrak{A}, \Phi^{\circ}\right)$ consisting of Hilbert space $\mathcal{G}=\mathcal{G}_{-} \otimes \mathcal{G}_{+}$embedding the Hilbert spaces $\mathfrak{f}=L_{\mu}^{2}$ by an isometry into $\mathcal{G}_{+}$, an algebra $\mathfrak{A}=\mathfrak{A}_{-} \otimes \mathfrak{A}_{+}$on $\mathcal{G}$ with an Abelian subalgebra $\mathfrak{A}_{-}=\mathfrak{C}$ generated by an observable (beable) $Y$ on $\mathcal{G}_{-}$, and a state-vector $\Phi^{\circ}=\Phi_{-}^{\circ} \otimes \Phi_{+}^{\circ} \in \mathcal{G}$ such that there exist a unitary operator $U$ on $\mathcal{H}=\mathfrak{h} \otimes \mathcal{G}$ which induces an endomorphism on the product algebra $\mathfrak{B}=\mathcal{B}(\mathfrak{h}) \otimes \mathfrak{A}$ in the sense $U^{\dagger} A U \in \mathfrak{B}$ for all $B \in \mathfrak{B}$, with

$$
\pi(\hat{g} \otimes \mathrm{B}):=\left(\mathrm{I} \otimes \Phi^{\circ}\right)^{\dagger} U^{\dagger}(\mathrm{B} \otimes g(Y)) U\left(\mathrm{I} \otimes \Phi^{\circ}\right)=\mathrm{M}\left[g V^{\dagger} \mathrm{B} V\right]
$$

for all $\mathrm{B} \in \mathcal{B}(\mathfrak{h})$ and measurable functions $g$ of $Y$, where $\mathrm{M}[B]=\int B(y) \mu_{y}$.

As it was pointed out in [3], it is always possible to achieve this dynamical invariance by extending the classical measurement apparatus' to an infinite auxiliary semi-classical system. It has been done in the previous section for the Schrödinger's atom and cat, and here we sketch this construction for the general unitary dilation (3.4). (The full proof is given in the Appendix 2.)

It consists of five steps. The first, preliminary step of a unitary dilation for the isometry $\mathrm{V}$ has been already described in the previous subsection.

Second, we construct the triple $\left(\mathcal{G}, \mathfrak{A}, \Phi^{\circ}\right)$. Denote by $\mathfrak{g}_{s}, s= \pm 0, \pm 1, \ldots$ (the indices \pm 0 are distinct and ordered as $-0<+0)$ the copies of the Hilbert space $\mathfrak{g}=\mathbb{C} \oplus \mathfrak{f}$ in the dilation (3.4) represented as the functional space $L_{\mu}^{2}$ on the values of $y$ including $y^{\circ}=0$, and $\mathbb{G}_{n}=\mathfrak{g}_{-n} \otimes \mathfrak{g}_{+n}, n \geq 0$. We define the Hilbert space of the past $\mathcal{G}_{-}$and the future $\mathcal{G}_{+}$as the state-vector spaces of semifinite discrete strings generated by the infinite tensor products $\Phi_{-}=\chi_{-0} \otimes \chi_{-1} \otimes \ldots$ and $\Phi_{+}=\chi_{+0} \otimes \chi_{+1} \otimes \ldots$ with all but finite number of $\chi_{s} \in \mathfrak{g}_{s}$ equal to the initial state $\chi_{s}^{\circ}$, the copies of $\chi^{\circ}=|0\rangle \in \mathfrak{g}$. Denoting by $\mathcal{A}_{s}$ the copies of the algebra $\mathcal{B}(\mathfrak{g})$ of bounded operators if $s \geq+0$, of the diagonal subalgebra $\mathcal{D}(\mathfrak{g})$ on $\mathfrak{g}$ if $s \leq-0$, and $\mathcal{A}_{n}=\mathcal{A}_{-n} \otimes \mathcal{A}_{+n}$ we construct the algebras of the past $\mathfrak{A}_{-}$and the future $\mathfrak{A}_{+}$and the whole algebra $\mathfrak{A} . \mathfrak{A}_{ \pm}$are generated on $\mathcal{G}_{ \pm}$respectively by the diagonal operators $\hat{f}_{-0} \otimes \hat{f}_{-1} \otimes \ldots$ and by $\mathrm{X}_{+0} \otimes \mathrm{X}_{+1} \otimes \ldots$ with all but finite number of $\hat{f}_{s} \in \mathcal{A}_{s}, s<0$ and $\mathrm{X}_{s} \in \mathcal{A}_{s}, s>0$ equal the identity operator $\hat{1}$ in $\mathfrak{g}$. Here $\hat{f}$ stands for the multiplication operator by a function $f$ of $y \in \mathbb{R}$, in particular, $\hat{y}$ is the multiplication by $y$, with the eigen-vector $\chi^{\circ}=|0\rangle$ corresponding to the eigen-value $y^{\circ}=0$. The Hilbert space $\mathcal{G}_{-} \otimes \mathcal{G}_{+}$identified with $\mathcal{G}=\otimes \mathbb{G}_{n}$, the decomposable algebra $\mathfrak{A}_{-} \otimes \mathfrak{A}_{+}$identified with $\mathfrak{A}=\otimes \mathcal{A}_{n}$ and the product vector $\Phi_{-} \otimes \Phi_{+}$identified with $\Phi=\otimes \phi_{n} \in \mathcal{G}$, where $\phi_{n}=\chi_{-n} \otimes \chi_{+n} \equiv \chi_{-n} \chi_{+n}$ with all $\chi_{s}=\chi^{\circ}$ stand as candidates for the triple $(\mathcal{G}, \mathfrak{A}, \Phi)$. Note that the eigen-vector $\Phi^{\circ}=\otimes \phi_{n}^{\circ}$ with all $\phi_{n}^{\circ}=\chi^{\circ} \otimes \chi^{\circ}$ corresponds to the initial eigen-state $y^{\circ}=0$ of all observables $Y_{ \pm n}=\hat{1}_{0} \otimes \ldots \otimes \hat{1}_{n-1} \otimes \hat{y}_{ \pm} \otimes \hat{1}_{n+1} \otimes$ in $\mathcal{G}$, where $\hat{1}=\hat{1}_{-} \otimes \hat{1}_{+}$, $\hat{y}_{-}=\hat{y} \otimes \hat{1}_{+}, \hat{y}_{+}=\hat{1}_{-} \otimes \hat{y}$ and $\hat{1}_{ \pm n}$ are the identity operators in $\mathfrak{g}_{ \pm n}$.

Third, we define the unitary evolution on the product space $\mathfrak{h} \otimes \mathcal{G}$ of the total system by

$$
U: \psi \otimes \chi_{-} \chi_{+} \otimes \chi_{-1} \chi_{+1} \cdots \mapsto \mathrm{W}\left(\psi \otimes \chi_{+}\right) \chi_{+1} \otimes \chi_{-} \chi_{+2} \cdots
$$

incorporating the right shift in $\mathcal{G}_{-}$, the left shift in $\mathcal{G}_{+}$and the conservative boundary condition $W: \mathfrak{h} \otimes \mathfrak{g}_{+} \rightarrow \mathfrak{h} \otimes \mathfrak{g}_{-}$given by the unitary dilation (3.4). We have 
obviously

$$
\left(\mathrm{I} \otimes\left\langle y_{-}, y_{+}, y_{-1}, y_{+1} \ldots\right|\right) U\left(\mathrm{I} \otimes\left|y_{-}^{0}, 0, y_{-1}^{0}, 0 \ldots\right\rangle\right)=\cdots \delta_{0}^{y_{+1}} \delta_{0}^{y_{+}} V\left(y_{-}\right) \delta_{y_{-}^{0}}^{y_{-1}} \delta_{y_{-1}^{0}}^{y_{-2}} \cdots
$$

so that the extended unitary operator $U$ still reproduces the reduction $V(y)$ in the result $y \neq y^{\circ}$ of the measurement $Y=Y_{-0}$ in a sequence $\left(Y_{-0}, Y_{+0}, Y_{-1} Y_{+1}, \ldots\right)$ with all other $y_{s}$ being zero $y^{\circ}=0$ with the probability one for the initial ground state $\Phi^{\circ}$ of the connected string.

Fourth, we prove the dynamical invariance $U^{\dagger}(\mathcal{B}(\mathfrak{h}) \otimes \mathfrak{A}) U \subseteq \mathcal{B}(\mathfrak{h}) \otimes \mathfrak{A}$ of the decomposable algebra of the total system, incorporating the measured quantum system $\mathcal{B}(\mathfrak{h})$ as the boundary between the quantum future (the right string considered as quantum, $\mathfrak{A}_{+}=\mathcal{B}\left(\mathcal{G}_{+}\right)$) with the classical past (the left string considered as classical, $\mathfrak{A}_{-}=\mathcal{D}\left(\mathcal{G}_{-}\right)$). This follows straightforward from the definition of $U$

$$
U^{\dagger}\left(\mathrm{B} \otimes \hat{g}_{-} \mathrm{X}_{+} \otimes \hat{g}_{-1} \mathrm{X}_{+1} \cdots\right) U=\hat{g}_{-1} \mathrm{~W}^{\dagger}\left(\mathrm{B} \otimes \hat{g}_{-}\right) \mathrm{W} \otimes \hat{g}_{-2} \mathrm{X}_{+} \cdots
$$

due to $\mathrm{W}^{\dagger}(\mathrm{B} \otimes \hat{g}) \mathrm{W} \in \mathcal{B}(\mathfrak{h}) \otimes \mathcal{B}(\mathfrak{g})$ for all $\hat{g} \in \mathcal{D}(\mathfrak{g})$. However this algebra representing the total algebra $\mathcal{B}(\mathfrak{h}) \otimes \mathfrak{A}$ on $\mathfrak{h} \otimes \mathcal{G}$ is not invariant under the inverse transformation, and there in no way to achieve the inverse invariance keeping $\mathfrak{A}$ decomposable as the requirement for statistical causality of quantum measurement if $\mathrm{W}(\mathrm{B} \otimes \mathrm{X}) \mathrm{W}^{\dagger} \notin \mathcal{B}(\mathfrak{h}) \otimes \mathcal{D}(\mathfrak{g})$ for some $\mathrm{B} \in \mathcal{B}(\mathfrak{h})$ and $\mathrm{X} \in \mathcal{B}(\mathfrak{g})$ :

$$
U\left(\mathrm{~B} \otimes \hat{g}_{-} \mathrm{X}_{+} \otimes \hat{g}_{-1} \mathrm{X}_{+1} \cdots\right) U^{\dagger}=\mathrm{W}\left(\mathrm{B} \otimes \mathrm{X}_{+}\right) \mathrm{W}^{\dagger} \mathrm{X}_{+1} \otimes \hat{g}_{-} \mathrm{X}_{+2} \cdots .
$$

And the fifth step is to explain on this dynamical model the decoherence phenomenon, irreversibility and causality by giving a constructive scheme in terms of equation for quantum predictions as statistical inferences by virtue of gaining the measurement information.

Because of the crucial importance of these realizations for developing understanding of the mathematical structure and interpretation of modern quantum theory, we need to analyze the mathematical consequences which can be drawn from such schemes.

3.1.3. Decoherence and quantum prediction. The analysis above shows that the dynamical realization of a quantum instantaneous measurement is possible in an infinitely extended system, but the discrete unitary group of unitary transformations $U^{t}, t \in \mathbb{N}$ with $U^{1}=U$ induces not a group of Heisenberg authomorphisms but an injective irreversible semigroup of endomorphisms on the decomposable algebra $\mathfrak{B}=\mathcal{B}(\mathfrak{h}) \otimes \mathfrak{A}$ of this system. However it is locally invertible on the center of the algebra $\mathfrak{A}$ in the sense that it reverses the shift dynamics on $\mathfrak{A}^{0]}$ :

$$
T_{-t}\left(\mathrm{I} \otimes Y_{s}\right) T_{t}:=\mathrm{I} \otimes Y_{s-t}=U^{t}\left(\mathrm{I} \otimes Y_{s}\right) U^{-t}, \quad \forall s \leq-0, t \in \mathbb{N} .
$$

Here $Y_{-n}=\hat{1}^{\otimes n} \otimes \hat{y}_{-} \otimes I_{n}$, where $I_{n}=\otimes_{k>n} \hat{1}_{k}$, and $T_{-t}=(T)^{t}$ is the power of the isometric shift $T: \Phi_{-} \mapsto \chi^{\circ} \otimes \Phi_{-}$on $\mathcal{G}_{-}$extended to the free unitary dynamics of the whole system as

$$
T: \psi \otimes \chi_{-} \chi_{+} \otimes \chi_{-1} \chi_{+1} \cdots \mapsto \psi \otimes \chi_{+} \chi_{+1} \otimes \chi_{-} \chi_{+2} \cdots
$$

The extended algebra $\mathfrak{B}$ is the minimal algebra containing all consistent events of the history and all admissible questions about the future of the open system 
under observation initially described by $\mathcal{B}(\mathfrak{h})$. Indeed, it contains all Heisenberg operators

$$
B(t)=U^{-t}(\mathrm{~B} \otimes I) U^{t}, \quad Y_{-}(t)=U^{-t}\left(\mathrm{I} \otimes Y_{-0}\right) U^{t}, \quad \forall t>0
$$

of $\mathrm{B} \in \mathcal{B}(\mathfrak{h})$, and these operators not only commute at each $t$, but also satisfy the nondemolition causality condition

$$
\left[B(t), Y_{-}(r)\right]=0, \quad\left[Y_{-}(t), Y_{-}(r)\right]=0, \quad \forall t \geq r \geq 0 .
$$

This follows from the commutativity of the Heisenberg string operators

$$
Y_{r-t}(t)=U^{-t}\left(\mathrm{I} \otimes Y_{r-t}\right) U^{t}=Y_{-}(r)
$$

at the different points $s=r-t<0$ coinciding with $Y_{s}(r-s)$ for any $s<0$ because of (3.7), and also from the commutativity with $\mathrm{B}(t)$ due to the simultaneous commutativity of all $Y_{s}(0)=\mathrm{I} \otimes \mathrm{Y}_{s}$ and $B(0)=\mathrm{B} \otimes I$. Thus all output Heisenberg operators $Y_{-}(r), 0<r \leq t$ at the boundary of the string can be measured simultaneously as $Y_{-n}(t)=Y_{-}(t-n)$ at the different points $n<t$, or sequentially at the point $s=-0$ as the commutative nondemolition family $Y_{0}^{t]}=\left(Y^{1}, \ldots, Y^{t}\right)$, where $Y^{r}=Y_{-}(r)$. This defines the reduced evolution operators

$$
V\left(t, y_{0}^{t]}\right)=V\left(y^{t}\right) V\left(y^{t-1}\right) \cdots V\left(y^{1}\right), \quad t>0
$$

of a sequential measurement in the system Hilbert space $\mathfrak{h}$ with measurement data $y_{0}^{t]}=\left\{(0, t] \ni r \mapsto y^{r}\right\}$. One can prove this (see the Appendix 2) using the filtering recurrency equation

$$
\psi\left(t, y_{0}^{t]}\right)=V\left(y_{t}\right) \psi\left(t-1, y_{0}^{t-1]}\right), \quad \psi(0)=\psi
$$

for $\psi\left(t, y_{0}^{t]}\right)=V\left(t, y_{0}^{t]}\right) \psi$ and for $\Psi(t)=U^{t}\left(\psi \otimes \Phi_{-} \otimes \Phi_{+}^{\circ}\right)$, where $\psi \in \mathfrak{h}$, and $V\left(y_{t}\right)$ is defined by

$$
\left(\mathrm{I} \otimes\left\langle y_{-\infty}^{t]}\right| \otimes\left\langle y_{t}^{\infty}\right|\right) U \Psi(t-1)=V\left(y_{t}\right) \psi\left(t-1, y_{0}^{t-1]}\right)\left\langle\delta_{0}^{y_{t}^{\infty}} y_{-\infty}^{0]}\right| \Phi_{-} .
$$

Moreover, any future expectations in the system, say the probabilities of the questions $F(t)=U^{-t}(F \otimes I) U^{t}, t \geq s$ given by orthoprojectors $F$ on $\mathfrak{h}$, can be statistically predicted upon the results of the past measurements of $Y_{-}(r), 0<r \leq t$ and initial state $\psi$ by the simple conditioning

$$
\operatorname{Pr}\left\{F(t) \mid E\left(\mathrm{~d} y^{1} \times \cdots \times \mathrm{d} y^{t}\right)\right\}=\frac{\operatorname{Pr}\left\{F(t) \wedge E\left(\mathrm{~d} y^{1} \times \cdots \times \mathrm{d} y^{t}\right)\right\}}{\operatorname{Pr}\left\{E\left(\mathrm{~d} y^{1} \times \cdots \times \mathrm{d} y^{t}\right)\right\}} .
$$

Here $E$ is the joint spectral measure for $Y^{1}, \ldots, Y^{t}$, and the probabilities in the numerator (and denominator) are defined as

$$
\left\|F(t) E\left(\mathrm{~d} y^{1} \times \cdots \times \mathrm{d} y^{t}\right)\left(\psi \otimes \Phi^{\circ}\right)\right\|^{2}=\left\|F \psi\left(t, y_{0}^{t]}\right)\right\|^{2} \mathrm{~d} \mu_{y^{1}} \cdots \mathrm{d} \mu_{y^{t}}
$$

(and for $F=\mathrm{I}$ ) due to the commutativity of $F(t)$ with $E_{-}$. This implies the usual sequential instrumental formula

$$
\langle\mathrm{B}\rangle\left(t, y_{0}^{r]}\right)=\frac{\psi^{\dagger} \pi\left(t, y_{0}^{r]}, \mathrm{B}\right) \psi}{\psi^{\dagger} \pi\left(t, y_{0}^{r]}, \mathrm{I}\right) \psi}=\mathrm{M}\left[\psi_{y_{0}^{t]}}^{\dagger}(t) \mathrm{B} \psi_{y_{0}^{t]}}(t) \mid y_{0}^{r]}\right]
$$


for the future expectations of B $(t)$ conditioned by $Y_{-}(1)=y^{1}, \ldots, Y_{-}(s)=y^{s}$ for any $t>r$. Here $\psi_{y_{0}^{t]}}(t)=\psi\left(t, y_{0}^{t]}\right) /\left\|\psi\left(t, y_{0}^{t]}\right)\right\|$, and

$$
\pi\left(t, y_{0}^{r]}, \mathrm{B}\right)=\int \cdots \int V\left(t, y_{0}^{r]}, y_{r}^{t]}\right)^{\dagger} \mathrm{B} V\left(t, y_{0}^{r]}, y_{r}^{t]}\right) \mathrm{d} \mu_{y^{r+1}} \cdots \mathrm{d} \mu_{y^{t}}
$$

is the sequential reduction map $V\left(t, y_{0}^{t]}\right)^{\dagger} \mathrm{B} V\left(t, y_{0}^{t]}\right)$ defining the prior probability distribution

$$
\mathrm{P}\left(\mathrm{d} y_{0}^{t]}\right)=\psi^{\dagger} \pi\left(t, y_{0}^{t]}, \mathrm{I}\right) \psi \mathrm{d} \mu_{y_{0}^{t]}}=\left\|\psi\left(t, y_{0}^{t]}\right)\right\|^{2} \mathrm{~d} \mu_{y^{1}} \cdots \mathrm{d} \mu_{y^{t}}
$$

integrated over $y_{r}^{t]}$ if these data are ignored for the quantum prediction of the state at the time $t>r$.

Note that the stochastic vector $\psi\left(t, y_{0}^{t]}\right)$, normalized as

$$
\int \cdots \int\left\|\psi\left(t, y_{0}^{t]}\right)\right\|^{2} \mathrm{~d} \mu_{y^{1}} \cdots \mathrm{d} \mu_{y^{t}}=1
$$

depends linearly on the initial state vector $\psi \in \mathfrak{h}$. However the posterior state vector $\psi_{y_{0}^{t]}}(t)$ is nonlinear, satisfying the nonlinear stochastic recurrency equation

$$
\psi_{y_{0}^{t]}}(t)=V_{y_{0}^{t-1]}}\left(t, y^{t}\right) \psi_{y_{0}^{t-1]}}(t-1), \quad \psi(0)=\psi,
$$

where $V_{y_{0}^{t-1]}}\left(t, y^{t}\right)=\left\|V\left(t-1, y_{0}^{t-1]}\right) \psi\right\| V\left(y^{t-1]}\right) /\left\|V\left(t, y_{0}^{t]}\right) \psi\right\|$.

In particular one can always realize in this way any sequential observation of the noncommuting operators $\mathrm{B}_{t}=\mathrm{e}^{i \mathrm{E} / \hbar} \mathrm{B}_{0} \mathrm{e}^{-i \mathrm{E} / \hbar}$ given by a selfadjoint operator $\mathrm{B}_{0}$ with discrete spectrum and the energy operator $\mathrm{E}$ in $\mathfrak{h}$. It corresponds to the sequential collapse given by $V(y)=\delta_{0}^{\mathrm{B}_{0}} \mathrm{e}^{-i \mathrm{E} / \hbar}$. Our construction suggests that any demolition sequential measurement can be realized as the nondemolition by the commutative family $Y_{-}(t), t>0$ with a common eigenvector $\Phi^{\circ}$ as the pointers initial state, satisfying the causality condition (3.8) with respect to all future Heisenberg operators B $(t)$. And the sequential collapse (3.10) follows from the usual Bayes formula for conditioning of the compatible observables due to the classical inference in the extended system. Thus, we have solved the sequential quantum measurement problem which can rigorously be formulated as

Given a sequential reduction family $V\left(t, y_{0}^{t]}\right), t \in \mathbb{N}$ of isometries resolving the filtering equation (3.9) on $\mathfrak{h}$ into $\mathfrak{h} \otimes \mathfrak{f}^{\otimes t}$, find a triple $(\mathcal{G}, \mathfrak{A}, \Phi)$ consisting of a Hilbert space $\mathcal{G}=\mathcal{G}_{-} \otimes \mathcal{G}_{+}$embedding all tensor products $\mathfrak{f}^{\otimes t}$ of the Hilbert spaces $\mathfrak{f}=L_{\mu}^{2}$ by an isometry into $\mathcal{G}_{+}$, an algebra $\mathfrak{A}=\mathfrak{A}_{-} \otimes \mathfrak{A}_{+}$on $\mathcal{G}$ with an Abelian subalgebra $\mathfrak{A}_{-}=\mathfrak{C}$ generated by a compatible discrete family $Y_{-\infty}^{0]}=\left\{Y_{s} s \leq 0\right\}$ of the observables (beables) $Y_{s}$ on $\mathcal{G}_{-}$, and a state-vector $\Phi^{\circ}=\Phi_{-}^{\circ} \otimes \Phi_{+}^{\circ} \in \mathcal{G}$ such that there exist a unitary group $U^{t}$ on $\mathcal{H}=\mathfrak{h} \otimes \mathcal{G}$ inducing a semigroup of endomorphisms $\mathfrak{B} \ni B \mapsto U^{-t} B U^{t} \in \mathfrak{B}$ on the product algebra $\mathfrak{B}=\mathcal{B}(\mathfrak{h}) \otimes \mathfrak{A}$ (3.) on $\mathfrak{A}$, with

$$
\pi^{t}\left(\hat{g}_{-t} \otimes \mathrm{B}\right)=\left(\mathrm{I} \otimes \Phi^{\circ}\right)^{\dagger} U^{-t}\left(g_{-t}\left(Y_{-t}^{0]}\right) \otimes \mathrm{B}\right) U^{t}\left(\mathrm{I} \otimes \Phi^{\circ}\right)=\mathrm{M}\left[g V(t)^{\dagger} \mathrm{B} V(t)\right]
$$

for any $\mathrm{B} \in \mathcal{B}(\mathfrak{h})$ and any operator $\hat{g}_{-t}=\hat{g}_{-t}\left(Y_{-t}^{0]}\right) \in \mathfrak{C}$ represented as the shifted function $\hat{g}_{-t}\left(y_{-t}^{0]}\right)=g\left(y_{0}^{t]}\right)$ of $Y_{-t}^{-0]}=\left(Y_{1-t}, \ldots, Y_{0}\right)$ on $\mathcal{G}$ by any measurable 
function $g$ of $y_{0}^{t]}=\left(y_{1}, \ldots, y_{t}\right)$ with arbitrary $t>0$, where

$$
\mathrm{M}\left[g V(t)^{\dagger} \mathrm{B} V(t)\right]=\int \cdots \int g\left(y_{0}^{t]}\right) V\left(t, y_{0}^{t]}\right)^{\dagger} \mathrm{B} V\left(t, y_{0}^{t]}\right) \mathrm{d} \mu_{y^{1}} \cdots \mathrm{d} \mu_{y^{t}} .
$$

Note that our construction of the solution to this problem admits also the time reversed representation of the sequential measurement process described by the isometry $\mathrm{V}$. The reversed system leaves in the same Hilbert space, with the same initial state-vector $\Phi^{\circ}$ in the auxiliary space $G$, however the reversed auxiliary system is described by the reflected algebra $\widetilde{\mathfrak{A}}=R \mathfrak{A} R$ where the reflection $R$ is described by the unitary flip-operator $R: \Phi_{-} \otimes \Phi_{+} \mapsto \Phi_{+} \otimes \Phi_{-}$on $\mathcal{G}=\mathcal{G}_{-} \otimes \mathcal{G}_{+}$. The past and future in the reflected algebra $\widetilde{\mathfrak{A}}=\mathfrak{A}_{+} \otimes \mathfrak{A}_{-}$are flipped such that its left subalgebra consists now of all operators on $\mathcal{G}_{-}, \widetilde{\mathfrak{A}}_{-}=\mathcal{B}(\mathcal{G}) \supset \mathfrak{A}_{-}$and its right subalgebra is the diagonal algebra $\widetilde{\mathfrak{A}}_{+}=\mathcal{D}\left(\mathcal{G}_{+}\right) \subset \mathfrak{A}_{+}$on $\mathcal{G}_{+}$. The inverse operators $U^{t}, t<0$ induce the reversed dynamical semigroup of the injective endomorphisms $B \mapsto U^{-t} B U^{t}$ which leaves invariant the algebra $\widetilde{\mathfrak{B}}=\mathcal{B}(\mathfrak{h}) \otimes \widetilde{\mathfrak{A}}$ but not $\mathfrak{B}$. The reversed canonical measurement process is described by another family $Y_{[+0}^{\infty}=\left(Y_{+t}\right)$ of commuting operators $Y_{+t}=R Y_{-t} R$ in $\widetilde{\mathfrak{A}}_{+}$, and the Heisenberg operators

$$
Y_{+}(t)=Y_{s}(t-s)=R Y_{-}(-t) R, \quad t<0, s>0,
$$

are compatible and satisfy the reversed causality condition

$$
\left[B(t), Y_{+}(r)\right]=0, \quad\left[Y_{+}(t), Y_{+}(r)\right]=0, \quad \forall t \leq r \leq 0 .
$$

It reproduces another, reversed sequence of the successive measurements

$$
V^{*}\left(t, y_{[t}^{0}\right)=V^{*}\left(y_{t}\right) V^{*}\left(y_{t+1}\right) \cdots V^{*}\left(y_{-1}\right), \quad t<0,
$$

where $V^{*}(y)=(\mathrm{I} \otimes\langle y|) \mathrm{W}^{-1}(\mathrm{I} \otimes|0\rangle)$ depends on the choice of the unitary dilation $\mathrm{W}$ of $\mathrm{V}$. In the case of the canonical dilation (3.4) uniquely defined up to the system evolution between the measurements, we obtain $V^{*}(y)=F(y) \mathrm{e}^{i \mathrm{E} / \hbar}$. If the system the Hamiltonian is time-symmetric, i.e. $\overline{\mathrm{E}}=\mathrm{E}$ in the sense $\mathrm{E} \bar{\psi}=$ $\overline{\mathrm{E} \psi}$ with respect to the complex (or another) conjugation in $\mathfrak{h}$, and if $\bar{F}(y)=$ $\mathrm{e}^{-i \mathrm{E} / \hbar} F(\tilde{y}) \mathrm{e}^{i \mathrm{E} / \hbar}$, where $y \mapsto \tilde{y}$ is a covariant flip, $\widetilde{\tilde{y}}=y$ (e.g. $\tilde{y}=y$, or reflection of the measurement data under the time reflection $t \mapsto-t)$, then $V^{*}(y)=\bar{V}(\tilde{y})$. This means that the reversed measurement process can be described as time-reflected direct measurement process under the $*$-conjugation $\psi^{*}(y)=\bar{\psi}(\tilde{y})$ in the space $\mathfrak{h} \otimes \mathfrak{f}$. And it can be modelled as the time reflected direct nondemolition process under the involution $J(\psi \otimes \Phi)=\bar{\psi} \otimes R \Phi^{*}$ induced by $\chi^{*}(y)=\bar{\chi}(\tilde{y})$ in $\mathfrak{g}$ with the flip-invariant eigen-value $y^{\circ}=0$ and $|0\rangle^{*}=|0\rangle$ corresponding to the real ground state $\chi^{\circ}(y)=\delta_{y}^{0}$.

Thus, the choice of time arrow, which is absolutely necessary for restoring statistical causality in quantum theory, is equivalent to a superselection rule. This corresponds to a choice of the minimal algebra $\mathfrak{B} \subset \mathcal{B}(\mathcal{H})$ generated by all admissible questions on a suitable Hilbert space $\mathcal{H}$ of the nondemolition representation for a process of the successive measurements. All consistent events should be drown from the center of $\mathfrak{B}$ : the events must be compatible with the questions, otherwise the propensities for the future cannot be inferred from the testing of the past. The decoherence is dynamically induced by a unitary evolution from any pure state on the algebra $\mathfrak{B}$ corresponding to the initial eigen-state for the measurement apparatus pointer which is described by the center of $\mathfrak{B}$. Moreover, the reversion of the 
time arrow corresponds to another choice of the admissible algebra. It can be implemented by a complex conjugation $J$ on $\mathcal{H}$ on the transposed algebra $\widetilde{\mathfrak{B}}=J \mathfrak{B} J$ . Note that the direct and reversed dynamics respectively on $\mathfrak{B}$ and on $\widetilde{\mathfrak{B}}$ are only endomorphic, and that the invertible authomorphic dynamics induced on the total algebra $\mathcal{B}(\mathcal{H})=\mathfrak{B} \vee \widetilde{\mathfrak{B}}$ does not reproduce the decoherence due to the redundancy of one of its part for a given time arrow $t$.

As Lawrence Bragg, another Nobel prize winner, once said, everything in the future is a wave, everything in the past is a particle.

\subsection{Quantum Jumps as a Boundary Value Problem.}

Have the 'jump' in the equations and not just the talk - J Bell.

Perhaps the closest to the truth was Bohr when he said that it 'must be possible so to describe the extraphysical process of the subjective perception as if it were in reality in the physical world'. He regarded the measurement apparatus, or meter, as a semiclassical object which interacts with the world in a quantum mechanical way but is essentially classical: it has only commuting observables - pointers. It relates the reality to a subjective observer as the classical part of the classical-quantum closed mechanical system. Thus Bohr accepted that not all the world is quantum mechanical, there is a classical part of the physical world, and we belong partly to this classical world.

In the previous section we have already shown how to realize this program as a discrete time boundary value problem of unitary interaction of the classical past with the quantum future. This however gives little for explanation of quantum jumps because any, even the Schrödinger unitary evolution in discrete time is described by jumps. Schrödinger himself tried unsuccessfully to derive the time continuous jumps from a boundary value problem between past and future for a maybe more general than his equation which would be relativistic and with infinite degrees of freedom. Here we shall see that in order to realize this program one should indeed consider a quantum field Dirac type boundary value problem, and the quantum stochastic models of jumps correspond to its ultrarelativistic limit.

In realizing this program I will start along the line suggested by John Bell [1] that the "development towards greater physical precision would be to have the 'jump' in the equations and not just the talk - so that it would come about as a dynamical process in dynamically defined conditions."

3.2.1. Stochastic decoherence equation. The generalized wave mechanics which enables us to treat the quantum spontaneous events, unstable systems and processes of time-continuous observation, or in other words, quantum mechanics with trajectories $\omega=\left(x_{t}\right)$, was discovered only quite recently, in [7, 9, 89. The basic idea of the theory is to replace the deterministic unitary Schrödinger propagation $\psi \mapsto \psi(t)$ by a linear causal stochastic one $\psi \mapsto \psi(t, \omega)$ which is not necessarily unitary for each history $\omega$, but unitary in the mean square sense, $\mathrm{M}\left[\|\psi(t)\|^{2}\right]=1$, with respect to a standard probability measure $\mu(\mathrm{d} \omega)$ for the measurable history subsets $\mathrm{d} \omega$. The unstable quantum systems can also be treated in the stochastic formalism by relaxing this condition by allowing the decreasing survival probabilities 
$\mathrm{M}\left[\|\psi(t)\|^{2}\right] \leq 1$. Due to this the positive measures

$$
\mathrm{P}(t, \mathrm{~d} \omega)=\|\psi(t, \omega)\|^{2} \mu(\mathrm{d} \omega), \quad \tilde{\mu}(\mathrm{d} \omega)=\lim _{t \rightarrow \infty} \mathrm{P}(t, \mathrm{~d} \omega)
$$

are normalized (if $\|\psi\|=1$ ) for each $t$, and are interpreted as the probability measure for the histories $\omega_{t}=\left\{(0, t] \ni r \mapsto x^{r}\right\}=x_{0}^{t]}$.of the output stochastic process $x^{t}$ with respect to the measure $\tilde{\mu}$. In the same way as the abstract Schrödinger equation can be derived from only unitarity of propagation, the abstract decoherence wave equation can be derived from the mean square unitarity in the form of a linear stochastic differential equation. The reason that Bohr and Schrödinger didn't derive such an equation despite their firm belief that the measurement process can be described 'as if it were in reality in the physical world' is that the appropriate (stochastic and quantum stochastic) differential calculus had not been yet developed early in that century. As classical differential calculus has its origin in classical mechanics, quantum stochastic calculus has its origin in quantum stochastic mechanics. A formal algebraic approach to this new calculus, which was developed in 77. 83, is presented in the Appendix 2.

Assuming that the superposition principle also holds for the stochastic waves such that $\psi(t, \omega)$ is given by a linear stochastic propagator $V(t, \omega)$, let us derive the general linear stochastic wave equation which preserves the mean-square normalization of these waves. Note that the abstract Schrödinger equation $i \hbar \partial_{t} \psi=\mathrm{E} \psi$ can also be derived as the general linear deterministic equation which preserves the normalization in a Hilbert space $\mathfrak{h}$. For the notational simplicity we shall consider here only the a finite-dimensional maybe complex trajectories $x^{t}=\left(x_{k}^{t}\right), k=1, \ldots, d$, the infinite-dimensional trajectories (fields) with even continuous index $k$ can be found elsewhere (e.g. in [7, 83]). It is usually assumed that the these $x_{k}^{t}$ as input stochastic processes have stationary independent increment $\mathrm{d} x_{k}^{t}=x_{k}^{t+\mathrm{d} t}-x_{k}^{t}$ with given expectations $\mathrm{M}\left[\mathrm{d} x_{k}^{t}\right]=\lambda_{k} \mathrm{~d} t$. The abstract linear stochastic decoherence wave equation is written then as

$$
\mathrm{d} \psi(t)+\left(\frac{\lambda^{2}}{2} \mathrm{R}+\frac{i}{\hbar} \mathrm{E}\right) \psi(t) \mathrm{d} t=\mathrm{L}^{k} \psi(t) \mathrm{d} x_{k}^{t}, \quad \psi(0)=\psi
$$

Here $\mathrm{E}$ is the system energy operator (the Hamiltonian of free evolution of the system), $\mathrm{R}=\mathrm{R}^{\dagger}$ is a selfadjoint operator describing a relaxation process in the system, $\mathrm{L}^{k}$ are any operators coupling the system to the trajectories $x_{k}$, and we use the Einstein summation rule $\mathrm{L}^{k} x_{k}=\sum \mathrm{L}^{k} x_{k} \equiv \mathrm{L} x, \lambda^{2}=\lambda^{k} \lambda_{k}$ with $\lambda^{k}=\bar{\lambda}_{k}$. In order to derive the relations between these operators which will imply the meansquare normalization of $\psi(t, \omega)$, let us rewrite this equation in the standard form

$$
\mathrm{d} \psi(t)+\mathrm{K} \psi(t) \mathrm{d} t=\mathrm{L}^{k} \psi(t) \mathrm{d} y_{k}^{t}, \quad \mathrm{~K}=\frac{\lambda^{2}}{2} \mathrm{R}+\frac{i}{\hbar} \mathrm{E}-\mathrm{L} \lambda,
$$

where $y_{k}^{t}=x_{k}^{t}-t \lambda_{k}$ are input noises as zero mean value independent increment processes with respect to the input probability measure $\mu$. Note that these noises will become the output information processes which will have dependent increments and correlations with the system with respect to the output probability measure $\tilde{\mu}=\mathrm{P}(\infty, \mathrm{d} \omega)$. If the Hilbert space valued stochastic process $\psi(t, \omega)$ is normalized in the mean square sense for each $t$, it represents a stochastic probability amplitude $\psi(t)$ as an element of an extended Hilbert space $\mathcal{H}_{0}=\mathfrak{h} \otimes L_{\mu}^{2}$. The stochastic process $t \mapsto \psi(t)$ describes a process of continual decoherence of the initial pure 
state $\rho(0)=P_{\psi}$ into the mixture

$$
\rho(t)=\int P_{\psi_{\omega}(t)} \mathrm{P}(t, \mathrm{~d} \omega)=\mathrm{M}\left[\psi(t) \psi(t)^{\dagger}\right]
$$

of the posterior states corresponding to $\psi_{\omega}(t)=\psi(t, \omega) /\|\psi(t, \omega)\|$, where M denotes mean with respect to the measure $\mu$. Assuming that the conditional expectation $\left\langle\mathrm{d} \bar{y}_{k}^{t} \mathrm{~d} y_{k}^{t}\right\rangle_{t}$ in

$$
\begin{aligned}
\left\langle\mathrm{d}\left(\psi^{\dagger} \psi\right)\right\rangle_{t} & =\left\langle\mathrm{d} \psi^{\dagger} \mathrm{d} \psi+\psi^{\dagger} \mathrm{d} \psi+\mathrm{d} \psi^{\dagger} \psi\right\rangle_{t} \\
& =\psi^{\dagger}\left(\mathrm{L}^{k \dagger}\left\langle\mathrm{d} \bar{y}_{k} \mathrm{~d} y_{k}\right\rangle_{t} \mathrm{~L}^{k}-\left(\mathrm{K}+\mathrm{K}^{\dagger}\right) \mathrm{d} t\right) \psi
\end{aligned}
$$

is $\mathrm{d} t$ (as in the case of the standard independent increment processes with $\bar{y}=y$ and $(\mathrm{d} y)^{2}=\mathrm{d} t+\varepsilon \mathrm{d} y_{t}$, see the Appendix 3), the mean square normalization in the differential form $\left\langle\mathrm{d}\left(\psi^{\dagger} \psi\right)\right\rangle_{t}=0\left(\right.$ or $\left\langle\mathrm{d}\left(\psi^{\dagger} \psi\right)\right\rangle_{t} \leq 0$ for the unstable systems) can be expressed [9, 11] as $\mathrm{K}+\mathrm{K}^{\dagger} \geq \mathrm{L}^{\dagger} \mathrm{L}$. In the stable case this defines the self-adjoint part of $\mathrm{K}$ as half of $\mathrm{L}^{\dagger} \mathrm{L}$, i.e.

$$
\mathrm{K}=\frac{1}{2} \mathrm{~L}^{\dagger} \mathrm{L}+\frac{i}{\hbar} \mathrm{H}, \quad \mathrm{L}^{\dagger} \mathrm{L}=\sum_{k} \mathrm{~L}^{k \dagger} \mathrm{L}^{k} \equiv \mathrm{L}_{k} \mathrm{~L}^{k}
$$

where $\mathrm{H}=\mathrm{H}^{\dagger}$ is the Schrödinger Hamiltonian in this equation when $\mathrm{L}=0$. One can also derive the corresponding Master equation

$$
\frac{\mathrm{d}}{\mathrm{d} t} \rho(t)+\mathrm{K} \rho(t)+\rho(t) \mathrm{K}^{\dagger}=\mathrm{L}^{k} \rho(t) \mathrm{L}_{k}
$$

for mixing decoherence of the initially pure state $\rho(0)=\psi \psi^{\dagger}$, as well as a stochastic nonlinear wave equation for the dynamical prediction of the posterior state vector $\psi_{\omega}(t)$, the normalization of $\psi(t, \omega)$ at each $\omega$.

3.2.2. Quantum jumps and unstable systems. Actually, there are two basic standard forms 89, 90] of such stochastic wave equations, corresponding to two basic types of stochastic integrators with independent increments: the Brownian standard type $x_{k}^{t} \simeq b_{k}^{t}$, and the Poisson standard type $x_{k}^{t} \simeq n_{k}^{t}$ with respect to the basic measure $\mu$, see the Appendix 3. We shall start with the Poisson case of the identical $n_{k}^{t}$ having all the expectations $\mathrm{M} n_{k}^{t}=\nu t$ and characterized by a very simple differential multiplication table

$$
\mathrm{d} n_{k}^{t}(\omega) \mathrm{d} n_{l}^{t}(\omega)=\delta_{l}^{k} \mathrm{~d} n_{l}^{t}(\omega)
$$

as it is for the only possible values $\mathrm{d} n_{k}^{t}=0,1$ of the counting increments at each time $t$. By taking all $x_{k}^{t}=n_{k}^{t} / \nu^{1 / 2}$ such that they have the expected rates $\lambda_{k}=\nu^{1 / 2}$ we can get the standard Poisson noises $y_{k}^{t}=x_{k}^{t}-\nu^{1 / 2} t \equiv m_{k}^{t}$ with respect to the input Poisson probability measure $\mu=\mathrm{P}_{m}$, described by the multiplication table

$$
\mathrm{d} m_{k} \mathrm{~d} m_{l}=\delta_{k}^{l}\left(\mathrm{~d} t+\nu^{-1 / 2} \mathrm{~d} m_{k}\right), \quad \mathrm{d} m_{k} \mathrm{~d} t=0=\mathrm{d} t \mathrm{~d} m_{k}
$$

Let us set now in our basic equation (3.11) the Hamiltonian $\mathrm{H}=\hbar\left(\mathrm{K}-\mathrm{K}^{\dagger}\right) / 2 i$ and the coupling operators $\mathrm{L}^{k}$ of the form

$$
\mathrm{L}^{k}=\lambda\left(\mathrm{C}^{k}-\mathrm{I}\right), \quad \mathrm{H}=\mathrm{E}+i \frac{\nu}{2}\left(\mathrm{C}^{k}-\mathrm{C}_{k}\right)
$$


with the coupling constant $\lambda=\nu^{1 / 2}$ and $\mathrm{C}^{k} \equiv \mathrm{C}_{k}^{\dagger}$ given by the collapse operators $\mathrm{C}_{k}$ (e.g. orthoprojectors, or contractions, $\mathrm{C}_{k}^{\dagger} \mathrm{C}_{k} \leq \mathrm{I}$ ). This corresponds to the stochastic decoherence equation of the form

$$
\mathrm{d} \psi(t)+\left(\frac{\nu}{2} \mathrm{R}+\frac{i}{\hbar} \mathrm{E}\right) \psi(t) \mathrm{d} t=\left(\mathrm{C}^{k}-\mathrm{I}\right) \psi(t) \mathrm{d} n_{k}^{t}, \quad \psi(0)=\psi,
$$

where $\mathrm{R} \geq \mathrm{C}^{\dagger} \mathrm{C}-\mathrm{I}$, or in the standard form (3.11) with $y_{k}^{t}=m_{k}^{t}$. In the stable case when $\mathrm{R}=\mathrm{C}^{\dagger} \mathrm{C}-\mathrm{I}$ this was derived from a unitary quantum jump model for counting nondemolition observation in [9, 91. It correspond to the linear stochastic decoherence Master-equation

$$
\mathrm{d} \varrho(t)+\left[\mathrm{G} \varrho(t)+\varrho(t) \mathrm{G}^{\dagger}-\nu \varrho(t)\right] \mathrm{d} t=\left[\mathrm{C}_{k} \varrho(t) \mathrm{C}^{k}-\varrho(t)\right] \mathrm{d} n_{k}^{t}, \quad \varrho(0)=\rho,
$$

for the not normalized (but normalized in the mean) density matrix $\varrho(t, \omega)$, where $\mathrm{G}=\frac{\nu}{2} \mathrm{C}_{k} \mathrm{C}^{k}+\frac{i}{\hbar} \mathrm{E}$ (it has the form $\psi(t, \omega) \psi(t, \omega)^{\dagger}$ in the case of a pure initial state $\left.\rho=\psi \psi^{\dagger}\right)$.

The nonlinear filtering equation for the posterior state vector

$$
\psi_{\omega}(t)=\psi(t, \omega) /\|\psi(t, \omega)\|
$$

has in this case the following form 90

$$
\mathrm{d} \psi_{\omega}+\left(\frac{\nu}{2}\left(\mathrm{C}_{k} \mathrm{C}^{k}-\sum\left\|\mathrm{C}_{k}^{\dagger} \psi_{\omega}\right\|^{2}\right)+\frac{i}{\hbar} \mathrm{E}\right) \psi_{\omega} \mathrm{d} t=\left(\mathrm{C}_{k}^{\dagger} /\left\|\mathrm{C}_{k}^{\dagger} \psi_{\omega}\right\|-\mathrm{I}\right) \psi_{\omega} \mathrm{d} n_{k, \omega}^{t, \rho},
$$

where $\|\psi\|=\langle\psi \mid \psi\rangle^{1 / 2}$ (see also [85 for the infinite-dimensional case). It corresponds to the nonlinear stochastic Master-equation

$$
\mathrm{d} \rho_{\omega}+\left[\mathrm{G} \rho_{\omega}+\rho_{\omega} \mathrm{G}^{\dagger}-\nu \rho_{\omega} \operatorname{TrC}^{k} \rho_{\omega} \mathrm{C}_{k}\right] \mathrm{d} t=\left[\mathrm{C}^{k} \rho_{\omega} \mathrm{C}_{k} / \operatorname{TrC}^{k} \rho_{\omega} \mathrm{C}_{k}-\rho\right] \mathrm{d} n_{k, \omega}^{t, \rho}
$$

for the posterior density matrix $\rho_{\omega}(t)$ which is the projector $P_{\omega}(t)=\psi_{\omega}(t) \psi_{\omega}(t)^{\dagger}$ for the pure initial state $\rho_{\omega}(0)=P_{\psi}$. Here $n_{k}^{\rho}(t, \omega)=n_{k, \omega}^{t, \rho(t)}$ are the output counting processes which are described by the history probability measure

$$
\mathrm{P}(t, \mathrm{~d} \omega)=\pi(t, \omega) \mu(\mathrm{d} \omega), \quad \pi(t, \omega)=\operatorname{Tr} \varrho(t, \omega)
$$

with the increment $\mathrm{d} n_{k}^{\rho}(t)$ independents of $n_{k}^{\rho}(t)$ under the condition $\rho_{\omega}(t)=\rho$ and the conditional expectations

$$
\mathrm{M}\left[\mathrm{d} n_{k}^{\rho}(t) \mid \rho_{\omega}(t)=\rho\right]=\nu \operatorname{TrC}_{k}^{\dagger} \rho \mathrm{C}_{k} \mathrm{~d} t
$$

which are $\nu\left\|\mathrm{C}_{k}^{\dagger} \psi\right\|^{2} \mathrm{~d} t$ for $\rho=P_{\psi}$. The derivation and solution of this equation was also considered in [84, and its solution was applied in quantum optics in 42, 46.

This nonlinear quantum jump equation can be written also in the quasi-linear form 89,90

$$
\mathrm{d} \psi_{\omega}(t)+\widetilde{\mathrm{K}}(t) \psi_{\omega}(t) \mathrm{d} t=\mathrm{L}^{k}(t) \psi_{\omega}(t) \mathrm{d} \tilde{m}_{k, \omega}^{t, \rho},
$$

where $\tilde{m}_{k}^{\rho}(t, \omega)=\tilde{m}_{k, \omega}^{t, \rho(t, \omega)}$ are the innovating martingales with respect to the output measure which is described by the differential

$$
\mathrm{d} \tilde{m}_{k}^{\rho}(t)=\nu^{-1 / 2}\left\|\mathrm{C}_{k}^{\dagger} \psi_{\omega}(t)\right\|^{-1} \mathrm{~d} n_{k}^{\rho}(t)-\nu^{1 / 2}\left\|\mathrm{C}_{k}^{\dagger} \psi_{\omega}(t)\right\| \mathrm{d} t
$$


with $\rho=P_{\psi}$ for $\psi=\psi_{\omega}(t)$ and the initial $\tilde{m}_{k}^{\rho}(0)=0$, the operator $\widetilde{\mathrm{K}}(t)$ similar to $\mathrm{K}$ has the form

$$
\widetilde{\mathrm{K}}(t)=\frac{1}{2} \widetilde{\mathrm{L}}(t)^{\dagger} \widetilde{\mathrm{L}}(t)+\frac{i}{\hbar} \widetilde{\mathrm{H}}(t)
$$

and $\widetilde{\mathrm{H}}(t), \widetilde{\mathrm{L}}(t)$ depend on $t$ (and $\omega$ ) through the dependence on $\psi=\psi_{\omega}(t)$ :

$$
\widetilde{\mathrm{L}}^{k}=\nu^{1 / 2}\left(\mathrm{C}_{k}^{\dagger}-\left\|\mathrm{C}_{k}^{\dagger} \psi\right\|\right), \quad \widetilde{\mathrm{H}}=\mathrm{E}+\frac{\nu}{2 i}\left(\mathrm{C}_{k}-\mathrm{C}_{k}^{\dagger}\right)\left\|\mathrm{C}_{k}^{\dagger} \psi\right\| .
$$

The latter form of the nonlinear filtering equation admits the central limit $\nu \rightarrow \infty$ corresponding to the standard Wiener case when $y_{k}^{t}=w_{k}^{t}$,

$$
\mathrm{d} w_{k} \mathrm{~d} w_{l}=\delta_{k}^{l} \mathrm{~d} t, \quad \mathrm{~d} w_{k} \mathrm{~d} t=0=\mathrm{d} t \mathrm{~d} w_{k},
$$

with respect to the limiting input Wiener measure $\mu$. If $\mathrm{L}^{k}$ and $\mathrm{H}$ do not depend on $\nu$, i.e. $\mathrm{C}_{k}$ and $\mathrm{E}$ depend on $\nu$ as

$$
\mathrm{C}_{k}=\mathrm{I}+\nu^{-1 / 2} \mathrm{~L}_{k}, \mathrm{E}=\mathrm{H}+\frac{\nu^{1 / 2}}{2 i}\left(\mathrm{~L}_{k}^{\dagger}-\mathrm{L}_{k}\right),
$$

then $\tilde{m}_{k}^{\rho}(t) \rightarrow \tilde{w}_{k}^{t}$, where the innovating diffusion process $\tilde{w}^{t}$ defined as

$$
\mathrm{d} \tilde{w}_{k}^{t}(\omega)=\mathrm{d} w_{k}^{t}(\omega)-2 \operatorname{Re}\left\langle\psi_{\omega}(t) \mid \mathrm{L}_{k}^{\dagger} \psi_{\omega}(t)\right\rangle \mathrm{d} t,
$$

are also standard Wiener processes but with respect to the output probability measure $\tilde{\mu}(\mathrm{d} \omega)=\mu(\mathrm{d} \tilde{\omega})$ due to

$$
\mathrm{d} \tilde{w}_{k} \mathrm{~d} \tilde{w}_{l}=\delta_{k}^{l} \mathrm{~d} t, \quad \mathrm{~d} \tilde{w}_{k} \mathrm{~d} t=0=\mathrm{d} t \mathrm{~d} \tilde{w}_{k} .
$$

.If $\left\|\psi_{\omega}(t)\right\|=1$ (which follows from the initial condition $\|\psi\|=1$ ), the stochastic operator-functions $\widetilde{\mathrm{L}}^{k}(t), \widetilde{\mathrm{H}}(t)$ defining the nonlinear filtering equation have the limits

$$
\widetilde{\mathrm{L}}^{k}=\mathrm{L}^{k}-\operatorname{Re}\left\langle\psi \mid \mathrm{L}^{k} \psi\right\rangle, \quad \widetilde{\mathrm{H}}=\mathrm{H}+\frac{i}{2}\left(\mathrm{~L}_{k}^{\dagger}-\mathrm{L}_{k}\right) \operatorname{Re}\left\langle\psi \mid \mathrm{L}^{k} \psi\right\rangle
$$

The corresponding nonlinear stochastic diffusion equation

$$
\mathrm{d} \psi_{\omega}(t)+\widetilde{\mathrm{K}}(t) \psi_{\omega}(t) \mathrm{d} t=\widetilde{\mathrm{L}}^{k}(t) \psi_{\omega}(t) \mathrm{d} \tilde{w}_{k}^{t}
$$

was first derived in the general multi-dimensional density-matrix form

$$
\mathrm{d} \rho_{\omega}+\left[\mathrm{K} \rho_{\omega}+\rho_{\omega} \mathrm{K}^{\dagger}-\mathrm{L}^{k} \rho_{\omega} \mathrm{L}_{k}\right] \mathrm{d} t=\left[\mathrm{L}^{k} \rho_{\omega}+\rho_{\omega} \mathrm{L}^{k \dagger}-\rho_{\omega} \operatorname{Tr}\left(\mathrm{L}^{k}+\mathrm{L}^{k \dagger}\right) \rho_{\omega}\right] \mathrm{d} \tilde{w}_{k}^{t}
$$

for the renormalized density matrix $\rho_{\omega}=\rho(\omega) / \operatorname{Tr} \rho(\omega)$ in [7, 13] from the microscopic reversible quantum stochastic unitary evolution models by the quantum filtering method. The general microscopic derivation for the case of multi-dimensional complete and incomplete measurements and solution in the linear-Gaussian case is given in 86. It has been recently applied in quantum optics 443, 44, 45, 47 for the description of counting, homodyne and heterodyne time-continuous measurements introduced in [91]. It has been shown in [95, 96] that the nondemolition observation of such a particle is described by filtering of the quantum noise which results in the continual collapse of any initial wave packet to the Gaussian stationary one localized at the position posterior expectation. 
The connection between the above diffusive nonlinear filtering equation and our linear decoherence Master-equation

$$
\mathrm{d} \varrho(t)+\left[\mathrm{K} \varrho(t)+\varrho(t) \mathrm{K}^{\dagger}-\mathrm{L}^{k} \varrho(t) \mathrm{L}_{k}\right] \mathrm{d} t=\left[\mathrm{L}^{k} \varrho(t)+\varrho(t) \mathrm{L}^{k \dagger}\right] \mathrm{d} w_{k}^{t}, \quad \varrho(0)=\rho,
$$

for the stochastic density operator $\varrho(t, \omega)$, defining the output probability density $\operatorname{Tr} \varrho(t, \omega)$, was well understood and presented in [12, 47, 48]. However it has also found an incorrect mathematical treatment in recent Quantum State Diffusion theory 92 based on the case $\varepsilon=0$ of our filtering equation (this particular nonlinear filtering equation is empirically postulated as the 'primary quantum state diffusion', and its more fundamental linear version $\mathrm{d} \psi+\mathrm{K} \psi \mathrm{d} t=\mathrm{L}^{k} \psi \mathrm{d} w_{k}$ is 'derived' in 92 simply by dropping the non-linear terms without appropriate change of the probability measures for the processes $\tilde{y}_{k}=\tilde{w}_{k}$ and $y_{k}=w_{k}$ ). The most general stochastic decoherence Master equation is given in the Appendix 3.

3.2.3. The derivation of jumps and localizations. Here we give the solution of the quantum jump problem for the stochastic model described by the equation (3.12) in the case $\mathrm{C}^{\dagger} \mathrm{C} \leq \mathrm{I}, \mathrm{R}=0$ which corresponds to the Hamiltonian evolution between the jumps with energy operator $\mathrm{E}$, and the jumps are cased only by the spontaneous decays or measurements. When $\mathrm{C}^{\dagger} \mathrm{C}=\mathrm{I}$, the quantum system certainly decays at the random moment of the jump $\mathrm{d} n_{k}^{t}=1$ to one of the $m$ products ending in the state $\mathrm{C}^{k} \psi /\left\|\mathrm{C}^{k} \psi\right\|$ from any state $\psi \in \mathfrak{h}$ with the probability $\left\|\mathrm{C}^{k} \psi\right\|^{2}$, or one of the measurement results $k=1, \ldots, m$ localizing the product is gained at the random moment of the spontaneous disintegration. The spontaneous evolution and its unitary quantum stochastic dilation was studied in details in [97]. When $\mathrm{C}^{\dagger} \mathrm{C}<\mathrm{I}$, the unstable system does not decay to one of the measurable products with the probability $\|\psi\|^{2}-\|\mathrm{C} \psi\|^{2}$, or no result is gained at the jump. This unstable spontaneous evolution and its unitary quantum stochastic dilation was considered in details for one dimensional case $m=1$ in 98 .

First, we consider the operator $\mathrm{C}$ as a construction (or isometry if $\mathrm{C}^{\dagger} \mathrm{C}=I$ ) from $\mathfrak{h}$ into $\mathfrak{h} \otimes \mathfrak{f}$, where $\mathfrak{f}=\mathbb{C}^{m}$. We dilate this $\mathrm{C}$ in the canonical way to the selfadjoint scattering operator

$$
\mathrm{S}=\left[\begin{array}{ll}
-\left(\mathrm{I}-\mathrm{C}^{\dagger} \mathrm{C}\right)^{1 / 2} & \mathrm{C}^{\dagger} \\
\mathrm{C} & \left(\mathrm{I} \otimes \hat{1}-\mathrm{CC}^{\dagger}\right)^{1 / 2}
\end{array}\right]
$$

where $\mathrm{I} \otimes \hat{1}$ is the identity operator in $\mathfrak{h} \otimes \mathfrak{f}$, and $\mathrm{C}^{\dagger}$ is the adjoint construction $\mathfrak{h} \otimes \mathfrak{f} \rightarrow \mathfrak{h}$ and $\mathrm{CC}^{\dagger}$ is a positive construction (orthoprojector $\mathrm{C}^{\dagger} \mathrm{C}=\mathrm{I}$ ) in this space. The unitarity $\mathrm{S}^{\dagger}=\mathrm{S}^{-1}$ of the operator $\mathrm{S}=\mathrm{S}^{\dagger}$ in the space $\mathfrak{g}=\mathbb{C} \oplus \mathfrak{f}=\mathbb{C}^{1+m}$ is easily proved as

$$
\mathrm{S}^{2}=\left[\begin{array}{ll}
\left(\mathrm{I}-\mathrm{C}^{\dagger} \mathrm{C}\right)+\mathrm{C}^{\dagger} \mathrm{C} & \mathrm{O} \\
\mathrm{O} & \mathrm{CC}^{\dagger}+\left(\mathrm{I} \otimes \hat{1}-\mathrm{CC}^{\dagger}\right)
\end{array}\right]=\left[\begin{array}{ll}
\mathrm{I} & \mathrm{O} \\
\mathrm{O} & \mathrm{I} \otimes \hat{1}
\end{array}\right]
$$

by use of the identity $\left(\mathrm{I} \otimes \hat{1}-\mathrm{CC}^{\dagger}\right)^{1 / 2} \mathrm{C}=\mathrm{C}\left(\mathrm{I}-\mathrm{C}^{\dagger} \mathrm{C}\right)^{1 / 2}$. The operators $\mathrm{C}^{k}=$ $\mathrm{S}_{0}^{k}$ are obtained from $\mathrm{S}$ as the partial matrix elements $(\mathrm{I} \otimes\langle k|) \mathrm{S}(\mathrm{I} \otimes|0\rangle)$ corresponding to the transition of the auxiliary system (pointer) form the initial state $|0\rangle=1 \oplus 0$ to one of the measured orthogonal states $0 \oplus|k\rangle, k=1, \ldots, m$ in the extended space $\mathfrak{g}$. 
Second, we consider two continuous seminfinite strings indexed by $s= \pm r$, where $r>0$ is any real positive number (one can think that $s$ is the coordinate on the right or left semistrings on the real line without the point $s=0$ ). Let us denote by $\mathfrak{g}^{\otimes}=\mathfrak{g}_{1} \otimes \mathfrak{g}_{2} \otimes \ldots \equiv \otimes \mathfrak{g}_{n}$ the infinite tensor product generated by $\otimes \chi_{n}$ with almost all components $\chi_{n} \in \mathfrak{g}_{n}=\mathfrak{g}$ equal to $\varphi=|0\rangle$ as they were defined in the Section 5.2. We shall consider right-continuous amplitudes $\Phi(v)$ with values in $\mathfrak{g}^{\otimes}$ for all infinite increasing sequences $v=\left\{r_{1}, r_{2}, \ldots\right\}, r_{n-1}<r_{n}$ having a finite number $n^{t}(v)=|v \cap[0, t)|$ of elements $r \in v^{t}$ in the finite intervals $[0, t)$ for all $t>0$ such that $f\left(r_{n}\right) \in \mathfrak{g}_{n}$ if $\Phi(v)=\otimes f\left(r_{n}\right)$. Let us also define the Hilbert space $L_{\mu}^{2} \otimes \mathfrak{g}^{\otimes}$ of the square-integrable functions $v \mapsto \Phi(v)$ in the sense

$$
\|\Phi\|^{2}=\int\langle\Phi(v) \mid \Phi(v)\rangle \mathrm{d} \mu_{v}^{\nu} \equiv \mathrm{M}\left(\|\Phi(\cdot)\|^{2}\right)<\infty
$$

with respect to the standard Poisson measure $\mu_{v}^{\nu}=\mathrm{P}_{m}$ with the constant intensity $\nu$ on $\mathbb{R}_{+}$. In other words we consider a countable number of the similar auxiliary systems (Schrödinger cats, bubbles or other pointers) described by the identical state spaces $\mathfrak{g}_{n}$ as independent randomly distributed on $\mathbb{R}_{+}$with the average number $\nu$ on any unit interval of the string. Let $\mathcal{G}_{ \pm}=L_{\mu}^{2} \otimes \mathfrak{g}_{ \pm}^{\otimes}$ be two copies of such space, one for the past, another for the future, and let $\mathcal{G}=L_{\mu}^{2} \otimes \mathbb{G}^{\otimes}$ with $\mathbb{G}=\mathfrak{g}_{-} \otimes \mathfrak{g}_{+}$be canonically identified with the space $\mathcal{G}_{-} \otimes \mathcal{G}_{+}$of square-integrable with $\mu_{-}^{\nu} \otimes \mu_{+}^{\nu}$ functions $\Phi\left(v_{-}, v_{+}\right)$having the values in $\mathfrak{g}_{-}^{\otimes} \otimes \mathfrak{g}_{+}^{\otimes}$. In particular, the ground state described by the constant function $\Phi^{\circ}(v)=\otimes \phi_{n}^{\circ}$, where $\phi^{\circ}=\varphi \otimes \varphi$, is identified with $\Phi_{-}^{\circ} \otimes \Phi_{+}^{\circ}$, where $\Phi_{ \pm}^{\circ}(v)=\otimes \varphi_{n}$. In order to maintain the quantum causality we shall select a decomposable algebra $\mathfrak{A}_{-} \otimes \mathfrak{A}_{+}$of the string with left string being classical, described by the commutative algebra $\mathfrak{A}_{-}=\mathcal{D}\left(\mathcal{G}_{-}\right)$, and the right string being quantum, described by the commutant $\mathfrak{A}_{+}$of the Abelian algebra $L_{\mu}^{\infty}$ of random scalar functions $f: v_{+} \mapsto \mathbb{C}$ represented by multiplication operators $\hat{f}$ on $\mathcal{G}_{+}$. More precisely, $\mathfrak{A}_{ \pm}$are the von Neumann algebras generated respectively on $\mathcal{G}_{ \pm}$by the functions $v_{ \pm} \mapsto A\left(v_{ \pm}\right)$with operator values $A\left(v_{+}\right) \in \mathcal{A}_{+}^{\otimes}$ and $A\left(v_{-}\right) \in \mathcal{A}_{-}^{\otimes}$, where $\mathcal{A}_{+}^{\otimes}=\otimes \mathcal{B}\left(\mathfrak{g}_{n}\right)$ is the algebra of all bounded operators $\mathcal{B}\left(\mathfrak{g}^{\otimes}\right)$ corresponding to $\mathcal{A}_{+}=\mathcal{B}\left(\mathbb{C}^{1+m}\right)$, and $\mathcal{A}_{-}^{\otimes}=\otimes \mathcal{D}\left(\mathfrak{g}_{n}\right)$ is its diagonal subalgebra $\mathcal{D}\left(\mathfrak{g}^{\otimes}\right)$ corresponding to $\mathcal{A}_{-}=\mathcal{D}\left(\mathbb{C}^{1+m}\right)$. The canonical triple $\left(\mathcal{G}, \mathfrak{A}, \Phi^{\circ}\right)$ is the appropriate candidate for the dynamical dilation of quantum jumps in the unstable system described by our spontaneous localization equation.

Third, we construct the time-continuous unitary group evolution which will dynamically induce the spontaneous jumps in the interaction representation at the boundary of the string. Let $T_{t}$ be the one parametric continuous unitary group on $\mathcal{G}=\mathcal{G}_{-} \otimes \mathcal{G}_{+}$describing the free evolution by right shifts $\Phi_{t}(\omega)=\Phi(\omega-t)$ when $\Phi\left(v_{-}, v_{+}\right)$is represented as $\Phi(\omega)$ with $\left(-v_{-}\right) \cup\left(+v_{+}\right) \subset \mathbb{R}$ for the two sided string parametrized by $\mathbb{R} \supset \omega$. (As in the discrete time case the corresponding Hilbert space $\mathcal{G}$ for such $\Phi$ will be denote as $\mathcal{G}^{0]} \otimes \mathcal{G}_{0}$ with $\mathcal{G}_{0}=\mathcal{G}_{+}$and $\mathcal{G}^{0]}$ obtained by the reflection of $\mathcal{G}_{-}$). This can be written as

$$
T_{t} \Phi\left(v_{-}, v_{+}\right)=\Phi\left(v_{-}^{t}, v_{+}^{t}\right)
$$

where $v_{ \pm}^{t}= \pm\left[\left(\left[\left(-v_{-}\right) \cup\left(+v_{+}\right)\right]-t\right) \cap \mathbb{R}_{ \pm}\right]$. Let us denote the selfadjoint generator of this free evolution on $\mathcal{G}$ by $P$ such that $T_{t}=\mathrm{e}^{-i P t / \hbar}$. This $P$ is the first 
order operator

$$
P \Phi\left(v_{-}, v_{+}\right)=\frac{\hbar}{i}\left(\sum_{r_{+} \in v_{+}} \frac{\partial}{\partial r_{+}}-\sum_{r_{-} \in v_{-}} \frac{\partial}{\partial r_{-}}\right) \Phi\left(v_{-}, v_{+}\right)
$$

on $\mathbb{R}_{+}$which is well defined on the differentiable functions $\Phi(v)$ which are constants for almost all $r \in v$. It is selfadjoint on a natural domain $\mathcal{D}_{0}$ in $\mathcal{G}$ corresponding to the boundary condition $\Phi\left(0 \sqcup v_{-}, v_{+}\right)=\Phi\left(v_{-}, 0 \sqcup v_{+}\right)$for all $v_{ \pm}>0$, where

$$
\Phi\left(0 \sqcup v_{-}, v_{+}\right)=\lim _{r \searrow 0} \Phi\left(r \sqcup v_{-}, v_{+}\right), \quad \Phi\left(v_{-}, 0 \sqcup v_{+}\right)=\lim _{r \searrow 0} \Phi\left(v_{-}, r \sqcup v_{+}\right)
$$

as induced by the continuity condition at $s=0$ on the whole $\mathbb{R}$. Here $r \sqcup v$ is adding a point $r \notin v$ to the ordered sequence $v=\left\{0, r_{1}, \ldots\right\}$, and $0 \sqcup v_{ \pm}$can be formally treated as adding two zeroth $s= \pm 0$ to $\left\{ \pm r_{1}, \pm r_{2}, \ldots\right\}$ such that $-r<-0<+0<+r$ for all $r>0$. Note that the Hamiltonian $-P$, not $+P$ corresponds to the right free evolution in positive arrow of time in which the states $\Phi\left(v_{+}\right)$describe the incoming "from the future" quantized waves, and the states $\Phi\left(v_{-}\right)$describe the outgoing "to the past" classical particles. This is a relativistic many particle Dirac type Hamiltonian on the half of the line $\mathbb{R}$ corresponding to zero particle mass and the orientation of spin along $\mathbb{R}$, and it has unbounded from below spectrum. However as we showed in 99, 100, the Heisenberg free evolution corresponding to this shift can be obtained as a WKB approximation in the ultrarelativistic limit $\langle p\rangle \rightarrow \infty$ of any free evolution with a positive single particle Hamiltonian, $\varepsilon(p)=|p|$ say. The unitary group evolution $U^{t}$ corresponding to the scattering interaction at the boundary with the unstable system which has its own free evolution described by the energy operator E can be obtained by resolving the following generalized Schrödinger equation

$$
\frac{\partial}{\partial t} \Psi^{t}\left(v_{-}, v_{+}\right)+\frac{i}{\hbar}(\mathrm{E} \otimes I) \Psi^{t}\left(v_{-}, v_{+}\right)=\left(\sum_{r \in v_{+}} \frac{\partial}{\partial r}-\sum_{r \in v_{-}} \frac{\partial}{\partial r}\right) \Psi^{t}\left(t, v_{-}, v_{+}\right)
$$

in the Hilbert space $\mathcal{H}=\mathfrak{h} \otimes \mathcal{G}$, with the following boundary condition

$$
\Psi^{t}\left(0 \sqcup v_{-}, v_{+}\right)=\mathrm{S}_{0} \Psi^{t}\left(v_{-}, 0 \sqcup v_{+}\right), \quad \forall t>0, v_{ \pm}>0 .
$$

Here $\mathrm{S}_{0}$ is the boundary action of $\mathrm{S}$ defined by $\mathrm{S}_{0} \Psi(0 \sqcup v)=\mathrm{S}[\psi \otimes \varphi] \otimes \Phi(v)$ on the products $\Psi(0 \sqcup v)=\psi \otimes \varphi \otimes \Phi(v)$. Due to the unitarity of the scattering matrix $\mathrm{S}$ this simply means $\left\|\Psi\left(0 \sqcup v_{-}, v_{+}\right)\right\|=\left\|\Psi\left(v_{-}, 0 \sqcup v_{+}\right)\right\|$, that is Dirac current has zero value at the boundary $r=0$. Note that this natural Dirac boundary condition corresponds to an unphysical discontinuity condition $\Psi(-0 \sqcup \omega)=(\mathrm{S} \otimes I) \Psi(+0 \sqcup \omega)$ at the origin $s=0$ when the doubled semi-string is represented as the two-sided string on $\mathbb{R}$.

Fourth, we have to solve this equation, or at least to prove that it has a unitary solution which induces the injective Heisenberg dynamics on the algebra $\mathfrak{B}=\mathcal{B}(\mathfrak{h}) \otimes \mathfrak{A}$ of the combined system with the required properties $U^{-t} \mathfrak{B} U^{t} \subseteq \mathfrak{B}$. The latter can be done by proof that the Hamiltonian in the equation (3.17) is selfadjoint on a natural domain corresponding to the perturbed boundary condition (3.18). It has been done by finding the appropriate domain for the perturbed Hamiltonian in the recent paper 101]. However we need a more explicit construction of the time continuous resolving operators $U^{t}: \Psi \mapsto \Psi^{t}$ for the equation (3.17). To obtain this 
we note that the this equation apart from the boundary condition coincides with the free evolution equation given by the Hamiltonian $-P$ up to the free unitary transformation $\mathrm{e}^{-i \mathrm{E} t / \hbar}$ in the initial space $\mathfrak{h}$. This implies that apart from the boundary the evolution $U^{t}$ coincides with $\mathrm{e}^{-i \mathrm{E} t / \hbar} \otimes T_{-t}$ such that the interaction evolution $U(t)=T_{t} U^{t}$, where $T_{t}$ is the shift extended trivially onto the component $\mathfrak{h}$ of $\mathcal{H}=\mathcal{G}^{0]} \otimes \mathfrak{h} \otimes \mathcal{G}_{0}$, is adapted in the sense

$$
U(t)\left(\Phi^{0]} \otimes \psi \otimes \varphi_{0}^{t]} \otimes \Phi_{t}\right)=\Phi^{0]} \otimes \psi(t) \otimes \Phi_{t},
$$

where $\psi(t)=\mathrm{W}_{0}^{t]}\left(\psi \otimes f_{0}^{t]}\right) \in \mathfrak{h} \otimes \mathcal{G}_{0}^{t]}$ for all $\psi \in \mathfrak{h}$ and $f_{0}^{t]} \in \mathcal{F}_{0}^{t]}$. Here we use the tensor product decomposition $\mathcal{G}_{0}=\mathcal{F}_{0}^{t]} \otimes \mathcal{G}_{t}$, where $\mathcal{F}_{0}^{t]}$ is Fock space generated by the products $f_{0}^{t]}(v)=\otimes_{r \in v} f(r)$ with finite $v \subset[0, t)$ and $f(r) \in \mathfrak{g}$ (we use the representation of $\mathcal{H}$ as the Hilbert space $\mathcal{G}^{0]} \otimes \mathfrak{h} \otimes \mathcal{G}_{0}$ for the two sided string on $\mathbb{R}$ with the measured system inserted at the origin $s=0$, and the notations $\mathcal{G}_{0}=\mathcal{G}_{+}$, $\mathcal{G}^{0]}=\mathcal{G}_{-}$, identifying $s=+r$ with $z=|s|$ for all $r>0$, and $s=-r$ with $z=-|s|$ including $r=0$ ). Moreover, in this interaction picture $U(t)$ is decomposable,

$$
[U(t) \Psi](\omega)=U(t, \omega) \Psi(\omega), \quad U(t, \omega)=I^{0]} \otimes \mathrm{W}_{0}^{t]}\left(\omega_{0}^{t]}\right) \otimes I_{t}
$$

as decomposable is each $\mathrm{W}_{0}^{t]}$ into the unitary operators $\mathrm{W}_{0}^{t]}(v)$ in $\mathfrak{h} \otimes \mathcal{F}_{0}^{t]}(v)$ with $\mathcal{F}_{0}^{t]}(v)=\otimes_{n=1}^{|v|} \mathfrak{g}_{n}$ for any finite $v \subset[0, t)$. The dynamical invariance $U^{-t} B U^{t} \in \mathfrak{B}$ of the algebra $\mathfrak{B}=\mathcal{B}(\mathfrak{h}) \otimes \mathfrak{A}$ for any positive $t>0$ under the Heisenberg transformations of the operators $B \in \mathfrak{B}$ induced by $U^{t}=T_{-t} U(t)$ simply follows from the right shift invariance $T_{t} \mathcal{B} T_{-t} \subseteq \mathfrak{B}$ of this algebra and $U(t, \omega)^{\dagger} \mathfrak{B}(\omega) U(t, \omega)=\mathfrak{B}(\omega)$ for each $\omega$ due to unitarity of $\mathrm{W}_{0}^{t]}(v)$ and the simplicity of the local algebras of future $\mathcal{B}_{0}^{t]}(v)=\mathcal{B}(\mathfrak{h}) \otimes \mathcal{A}_{+}^{\otimes|v|}$ corresponding to $\mathcal{A}_{+}=\mathcal{B}(\mathfrak{g})$.

And finally we can find the nondemolition processes $N_{k}^{t}, k=1, \ldots, m$ which count the spontaneous disintegrations of the unstable system to one of the measured products $k=1, \ldots, m$, and with $N_{0}^{t}$ corresponding to the unobserved jumps. These are given on the space $\mathcal{G}_{-}$as the sums of the orthoprojectors $|k\rangle\langle k| \in \mathfrak{g}$ corresponding to the arriving particles at the times $r_{n} \in v_{-}$up to the time $t$ :

$$
N_{k}^{t}\left(v_{-}, v_{+}\right)=\mathrm{I} \otimes \sum_{n=1}^{n^{t}\left(v_{-}\right)} \mathrm{I}_{0}^{n-1]} \otimes\left(|k\rangle\langle k| \otimes \mathrm{I}_{+}\right) \otimes I_{n} .
$$

Due to commutativity of this compatible family with all operators $\mathrm{B} \otimes I$ of the unstable system, the Heisenberg processes $N_{k}(t)=U^{-t} N_{k}^{t} U^{t}$ satisfies the nondemolition causality condition. The independent increment quantum nondemolition process with zero initial expectations corresponding to $y_{k}^{t}=m_{k}^{t}$ in the standard jump-decoherence equation (3.11) then is given by

$$
Y_{k}^{t}=\nu^{-1 / 2}\left(N_{k}^{t}-\nu t\right)=X_{k}^{t}-\lambda t
$$

where $X_{k}^{t}=\lambda^{-1 / 2} N_{k}^{t}$ with the coupling constant $\lambda=\nu^{1 / 2}$. Hence the quantum jumps, decoherence and spontaneous localization are simply derived from this dynamical model as the results of inference, or quantum filtering without the projection postulate by simple conditioning. The statistical equivalence of the nondemolition countings $N_{k}^{t}$ in the Schrödinger picture $\Psi^{t}=U^{t}\left(\psi \otimes \Phi^{\circ}\right)$ of this continuous unitary evolution model with fixed initial ground state $\Phi^{\circ}=\Phi_{-}^{\circ} \otimes \Phi_{+}^{\circ}$ and the stochastic quantum reduction model based on the spontaneous jump equation (3.12) 
for an unstable system corresponding to $\mathrm{R}=0$ and $\mathrm{C}^{\dagger} \mathrm{C} \leq \mathrm{I}$ was proved in the interaction representation picture in 97,857 .

Note that before the interaction the probability to measure any of these products is zero in the initial states $\Phi^{\circ}\left(v_{-}, v_{+}\right)=\otimes \phi_{n}^{\circ}$ as all $\phi_{n}^{\circ}=|0\rangle \otimes|0\rangle$. The maximal decreasing orthoprojector

$$
E_{t}\left(v_{-}, v_{+}\right)=\mathrm{I} \otimes E_{1}(0) \otimes \ldots \otimes E_{n^{t}\left(v_{-}\right)}(0) \otimes I_{n^{t}\left(v_{-}\right)}, \quad E_{n}=|0\rangle\langle 0| \otimes \mathrm{I}_{+},
$$

which is orthogonal to all product countings $N_{k}^{t}, k=1, \ldots, m$, is called the survival process for the unstable system.

Thus, the quantum jumps problem has been solved as the time-continuous quantum boundary-value problem formulated as

Given a reduction family $V(t, \omega)=V\left(t, m_{0}^{t]}\right), t \in \mathbb{R}_{+}$of isometries on $\mathfrak{h}$ into $\mathfrak{h} \otimes L_{\mu}^{2}$ resolving the quantum jump equation (3.19) with respect to the input probability measure $\mu=\mathrm{P}_{m}$ for the standard Poisson noises $m_{k}^{t}$ find a triple $(\mathcal{G}, \mathfrak{A}, \Phi)$ consisting of a Hilbert space $\mathcal{G}=\mathcal{G}_{-} \otimes \mathcal{G}_{+}$embedding the Poisson Hilbert space $L_{\mu}^{2}$ by an isometry into $\mathcal{G}_{+}$, an algebra $\mathfrak{A}=\mathfrak{A}_{-} \otimes \mathfrak{A}_{+}$on $\mathcal{G}$ with an Abelian subalgebra $\mathfrak{A}_{-}$generated by a compatible continuous family $Y_{-\infty}^{0]}=\left\{Y_{k}^{s}, k=1, \ldots, d, s \leq 0\right\}$ of observables (beables) on $\mathcal{G}_{-}$, and a state-vector $\Phi^{\circ}=\Phi_{-}^{\circ} \otimes \Phi_{+}^{\circ} \in \mathcal{G}$ such that there exist a time continuous unitary group $U^{t}$ on $\mathcal{H}=\mathfrak{h} \otimes \mathcal{G}$ inducing a semigroup of endomorphisms $\mathfrak{B} \ni B \mapsto U^{-t} B U^{t} \in \mathfrak{B}$ on the product algebra $\mathfrak{B}=\mathcal{B}(\mathfrak{h}) \otimes \mathfrak{A}$, with

$$
\begin{aligned}
\pi^{t}\left(\hat{g}_{-t} \otimes \mathrm{B}\right): & =\left(\mathrm{I} \otimes \Phi^{\circ}\right)^{\dagger} U^{-t}\left(\mathrm{~B} \otimes g_{-t}\left(Y_{-t}^{0]}\right)\right) U^{t}\left(\mathrm{I} \otimes \Phi^{\circ}\right) \\
= & \int g\left(y_{0}^{t]}\right) V\left(t, y_{0}^{t]}\right)^{\dagger} \mathrm{B} V\left(t, y_{0}^{t]}\right) \mathrm{d} \mathrm{P}_{m} \equiv \mathrm{M}\left[g V(t)^{\dagger} \mathrm{B} V(t)\right]
\end{aligned}
$$

for any $\mathrm{B} \in \mathcal{B}(\mathfrak{h})$ and any operator $\hat{g}_{-t}=\hat{g}_{-t}\left(Y_{-t}^{0]}\right) \in \mathfrak{C}$ represented as the shifted functional $\hat{g}_{-t}\left(y_{-t}^{0]}\right)=g\left(y_{0}^{t]}\right)$ of $Y_{-t}^{-0]}=\left\{Y^{s}: s \in(-t, 0]\right\}$ on $\mathcal{G}$ by any measurable functional $g$ of $y_{0}^{t]}=\left\{y^{r}: r \in(0, t]\right\}$ with arbitrary $t>0$.

Note that despite strong continuity of the unitary group evolutions $T_{t}$ and $U^{t}$, the interaction evolution $U(t, \omega)$ is time-discontinuous for each $\omega$. It is defined as $U(t)=I^{0]} \otimes W(t)$ for any positive $t$ by the stochastic evolution $W(t)=\mathrm{W}_{0}^{t]} \otimes I_{t}$ resolving the Schrödinger unitary jump equation [97, 85]

$$
\mathrm{d} \Psi_{0}(t, v)+\frac{i}{\hbar}(\mathrm{E} \otimes I) \Psi_{0}(t, v) \mathrm{d} t=(S-I)_{t}(v) \Psi_{0}(t, v) \mathrm{d} n^{t}(v)
$$

on $\mathcal{H}_{0}=\mathfrak{h} \otimes \mathcal{G}_{0}$ as $\Psi_{0}(t, v)=W(t, v) \Psi_{0}$. Here $L_{t}(v)=\mathrm{L}_{n^{t}(v)} \otimes I_{t}$ is the adapted generator $\mathrm{L}=\mathrm{S}-\mathrm{I}$ which is applied only to the system and the $n$ th particle with the number $n=n^{t}(v)$ on the right semistring as the operator $\mathrm{L}_{n}=\mathrm{T}_{0}^{n] \dagger}\left(\mathrm{I}_{0}^{n-1]} \otimes \mathrm{L}_{0}\right) \mathrm{T}_{0}^{n]}$ obtained from $\mathrm{L}_{0}=\mathrm{TLT}^{\dagger}$ by the transposition operator $\mathrm{T}(\psi \otimes \chi)=\chi \otimes \psi$ generating $\mathrm{T}_{0}^{n]}\left(\psi \otimes \chi_{0}^{n]}\right)=\chi_{0}^{n]} \otimes \psi$ on $\mathfrak{h} \otimes \mathfrak{g}_{0}^{n]}$ by the recurrency

$$
\mathrm{T}_{0}^{n]}=\left(\mathrm{I}_{0}^{n-1]} \otimes \mathrm{T}\right)\left(\mathrm{T}_{0}^{n-1]} \otimes \mathrm{I}\right), \quad \mathrm{T}_{0}^{0]}=\mathrm{I} .
$$


The operator $\mathrm{W}_{0}^{t]}$ can be explicitly found from the equivalent stochastic integral equation

$$
\mathrm{W}_{0}^{t]}(v)=\left(\mathrm{e}^{-i \mathrm{E} t / \hbar} \otimes \mathrm{I}_{0}^{t]}\right)+\sum_{r \in v \cap\{0, t)}\left(\mathrm{e}^{i \mathrm{E}(r-t) / \hbar} \otimes \mathrm{I}_{0}^{t]}\right)(\mathrm{S}-\mathrm{I})_{n^{r}}\left(\mathrm{~W}_{0}^{r]} \otimes \mathrm{I}_{r}^{t]}\right)
$$

with $\mathrm{W}_{0}^{0]}=\mathrm{I}$. Indeed, the solution to this integral equation can be written for each $v$ in terms of the finite chronological product of unitary operators as in the discrete time case iterating the following recurrency equation

$$
\mathrm{W}_{0}^{t]}(v)=\mathrm{e}^{-i \mathrm{E} t / \hbar} \mathrm{S}\left(t_{n}\right)\left(\mathrm{W}_{0}^{\left.t_{n}\right]}(v) \otimes \mathrm{I}\right), \quad \mathrm{W}_{0}^{0]}(v)=\mathrm{I},
$$

where $\mathrm{S}\left(t_{n}\right)=\mathrm{e}^{i \mathrm{E} t_{n} / \hbar} \mathrm{S}_{n} \mathrm{e}^{-i \mathrm{E} t_{n} / \hbar}$ with $n=n_{t}(v)$. From this we can also obtain the corresponding explicit formula 97, 85,

$$
V(t, v .)=\mathrm{e}^{-i \mathrm{E} t / \hbar} \mathrm{C}^{k_{n}}\left(t_{n}\right)\left(V\left(t_{n}, v\right) \otimes \mathrm{I}\right), \quad \mathrm{V}(v .)=\mathrm{I},
$$

resolving the reduced stochastic equation (3.12) as $\psi(t)=V(t) \psi$ for $\mathrm{R}=\mathrm{O}$ and any sequence $v$. of pairs $\left(r_{n}, k_{n}\right)$ with increasing $\left\{r_{n}\right\}$, where $n=n^{t}(v$.) is the maximal number in $\left\{r_{n}\right\} \cap[0, t)$.

\subsection{Continuous Trajectories and State Diffusion.}

Quantum mechanics itself, whatever its interpretation, does not account for the transition from 'possible to the actual' - Heisenberg.

Schrödinger believed that all quantum problems including the interpretation of measurement should be formulated in continuous time in the form of differential equations. He thought that the measurement problem would have been resolved if quantum mechanics had been made consistent with relativity theory and the time had been treated appropriately. However Einstein and Heisenberg did not believe this, each for his own reasons.

Although Schrödinger did not succeed to find the 'true Schrödinger equation' to formulate the boundary value problem for such transitions, the analysis of the phenomenological stochastic models for quantum spontaneous jumps in the unstable systems proves that Schrödinger was right. However Heisenberg was also right as in order to make an account for these transitions by filtering the actual past events simply as it is done in classical statistics, the corresponding ultrarelativistic Dirac type boundary value problem (3.17), (3.18) must be supplemented by future-past superselection rule for the total algebra as it follows from the nondemolition causality principle [2]. This principle cannot be formulated in quantum mechanics as it involves infinitely many degrees of freedom, and it has not been formulated even in the orthodox quantum field theory.

Here we shall deal with quantum noise models which allow to formulate the most general stochastic decoherence equation which was derived in 116. We shall start with a simple quantum noise model and show that it allows to prove the 'true Heisenberg principle' in the form of an uncertaincy relation for measurement errors and dynamical perturbations. The discovery of quantum thermal noise and its white-noise approximations lead to a profound revolution not only in modern physics but also in contemporary mathematics comparable with the discovery of differential calculus by Newton (for a feature exposition of this, accessible for 
physicists, see [76], the complete theory, which was mainly developed in the 80's 4. 77, 78, 79, is sketched in the Appendix 2)

3.3.1. The true Heisenberg principle. The first time continuous solution of the quantum measurement problem [4 was motivated by analogy with the classical stochastic filtering problem which obtains the prediction of future for an unobservable dynamical process $x(t)$ by time-continuous measuring of another, observable process $y(t)$. Such problems were first considered by Wiener and Kolmogorov who found the solutions in the form of a causal spectral filter for a liner estimate $\hat{x}(t)$ of $x(t)$ which is optimal only in the stationary Gaussian case. The complete solution of this problem was obtained by Stratonovich 88 in 1958 who derived a stochastic filtering equation giving the posterior expectations $\hat{x}(t)$ of $x(t)$ in the arbitrary Markovian pair $(x, y)$. This was really a break through in the statistics of stochastic processes which soon found many applications, in particular for solving the problems of stochastic control under incomplete information (it is possible that this was one of the reasons why the Russians were so successful in launching the rockets to the Moon and other planets of the Solar system in 60s).

If $X(t)$ is an unobservable Heisenberg process, or vector of such processes $X_{k}(t)$, $k=1, \ldots, d$ which might have even no prior trajectories as the Heisenberg coordinate processes of a quantum particle say, and $Y(t)$ is an actual observable quantum processes, i.e. a sort of Bell's beable describing the vector trajectory $y(t)$ of the particle in a cloud chamber say, why don't we find the posterior trajectories by deriving and solving a filtering equation for the posterior expectations $\hat{x}(t)$ of $X(t)$ or any other function of $X(t)$, defining the posterior trajectories $x\left(t, y_{0}^{t]}\right)$ in the same way as we do it in the classical case? If we had a dynamical model in which such beables existed as a nondemolition process, we could solve this problem simply by conditioning as the statistical inference problem, predicting the future knowing a history, i.e. a particular trajectory $y(r)$ up to the time $t$. This problem was first considered and solved by finding a nontrivial quantum stochastic model for the Markovian Gaussian pair $(X, Y)$. It corresponds to a quantum open linear system with linear output channel, in particular for a quantum oscillator matched to a quantum transmission line [4, 5]. By studying this example, the nondemolition condition

$$
\left[X_{k}(s), Y(r)\right]=0, \quad[Y(s), Y(r)]=0 \quad \forall r \leq s
$$

was first found, and this allowed the solution in the form of the causal equation for $x\left(t, y_{0}^{t]}\right)=\langle X(t)\rangle_{y_{0}^{t]}}$.

Let us describe this exact dynamical model of the causal nondemolition measurement first in terms of quantum white noise for a quantum nonrelativistic particle of mass $m$ which is conservative if not observed, in a potential field $\phi$. But we shall assume that this particle is under a time continuous indirect observation which is realized by measuring of its Heisenberg position operators $Q^{k}(t)$ with additive random errors $e^{k}(t)$ :

$$
Y^{k}(t)=Q^{k}(t)+e^{k}(t), \quad k=1, \ldots, d .
$$

We take the simplest statistical model for the error process $e(t)$, the white noise model (the worst, completely chaotic error), assuming that it is a classical Gaussian 
white noise given by the first momenta

$$
\left\langle e^{k}(t)\right\rangle=0, \quad\left\langle e^{k}(s) e^{l}(r)\right\rangle=\sigma_{e}^{2} \delta(s-r) \delta_{l}^{k} .
$$

The components of measurement vector-process $Y(t)$ should be commutative, satisfying the causal nondemolition condition with respect to the noncommutative process $Q(t)$ (and any other Heisenberg operator-process of the particle), this can be achieved by perturbing the particle Newton-Erenfest equation:

$$
m \frac{\mathrm{d}^{2}}{\mathrm{~d} t^{2}} Q(t)+\nabla \phi(Q(t))=f(t) .
$$

Here $f(t)$ is vector-process of Langevin forces $f_{k}$ perturbing the dynamics due to the measurement, which are also assumed to be independent classical white noises

$$
\left\langle f_{k}(t)\right\rangle=0, \quad\left\langle f_{k}(s) f_{l}(r)\right\rangle=\sigma_{f}^{2} \delta(s-r) \delta_{l}^{k} .
$$

In classical measurement and filtering theory the white noises $e(t), f(t)$ are usually considered independent, and the intensities $\sigma_{e}^{2}$ and $\sigma_{f}^{2}$ can be arbitrary, even zeros, corresponding to the ideal case of the direct unperturbing observation of the particle trajectory $Q(t)$. However in quantum theory corresponding to the standard commutation relations

$$
Q(0)=\mathrm{Q}, \quad \frac{\mathrm{d}}{\mathrm{d} t} Q(0)=\frac{1}{m} \mathrm{P}, \quad\left[\mathrm{Q}^{k}, \mathrm{P}_{l}\right]=i \hbar \delta_{l}^{k} \mathrm{I}
$$

the particle trajectories do not exist such that the measurement error $e(t)$ and perturbation force $f(t)$ should satisfy a sort of uncertainty relation. This "true Heisenberg principle" had never been mathematically proved before the discovery (4) of quantum causality in the form of nondemolition condition of commutativity of $Q(s)$, as well as any other process, the momentum $P(t)=m \dot{Q}(t)$ say, with all $Y(r)$ for $r \leq s$. As we showed first in the linear case [4, 5], and later even in the most general case [86], these conditions are fulfilled if and only if $e(t)$ and $f(t)$ satisfy the canonical commutation relations

$$
\left[e^{k}(r), e^{l}(s)\right]=0,\left[e^{k}(r), f_{l}(s)\right]=\frac{\hbar}{i} \delta(r-s) \delta_{l}^{k},\left[f_{k}(r), f_{l}(s)\right]=0 .
$$

From this it follows, in a similar way as it was done in the Sec. 3.1, that the pair $(e, f)$ satisfy the uncertainty relation $\sigma_{e} \sigma_{f} \geq \hbar / 2$. This inequality constitutes the precise formulation of the true Heisenberg principle for the square roots $\sigma_{e}$ and $\sigma_{f}$ of the intensities of error $e$ and perturbation $f$ : they are inversely proportional with the same coefficient of proportionality, $\hbar / 2$, as for the pair $(\mathrm{Q}, \mathrm{P})$. Note that the canonical pair $(e, f)$ called quantum white noise cannot be considered classically, despite the fact that each process $e$ and $f$ separately can. This is why we need a quantum-field representation for the pair $(e, f)$, and the corresponding quantum stochastic calculus. Thus, a generalized matrix mechanics for the treatment of quantum open systems under continuous nondemolition observation and the true Heisenberg principle was discovered 20 years ago only after the invention of quantum white noise in [4]. The nondemolition commutativity of $Y(t)$ with respect to the Heisenberg operators of the open quantum system was later rediscovered for the output of quantum stochastic fields in 78 .

Let us outline the exact quantum stochastic model [7, 86 for a quantum particle of mass $m$ in a potential $\phi$ under indirect observation of the positions $Q^{k}$ by measuring $Y_{k}$. We define the output process as a quantum stochastic Heisenberg 
transformation $Y_{k}^{t}=W(t)^{\dagger}\left(\mathrm{I} \otimes \hat{y}_{k}^{t}\right) W(t)$ for a time-continuous quantum stochastic unitary evolution $W(t)$ in a similar way as we did in the discrete case, see the Appendix 2. It has been shown in [7, 86] that $W(t)$ is the resolving family for an appropriate quantum stochastic Schrödinger equation (See the equation (3.23) below). It induces the following quantum stochastic Heisenberg output equation

$$
\mathrm{d} Y_{k}^{t}=2 \lambda Q^{k}(t) \mathrm{d} t+\mathrm{d} \hat{w}_{k}^{t} \equiv X_{k}(t) \mathrm{d} t+\mathrm{d} \hat{w}_{k}^{t},
$$

where $\lambda$ is a coupling constant, or a diagonal matrix $\lambda=\left[\lambda_{k} \delta_{k}^{i}\right]$ defining different accuracies of an indirect measurement it time of $\mathrm{Q}^{k}$. Here $X(t)=W(t)^{\dagger}\left(\mathrm{X} \otimes I_{0}\right) W(t)$ are the system Heisenberg operators for $\mathrm{X}_{k}=2(\lambda Q)^{k}, I_{0}$ is the identity operator in the Fock space $\mathcal{F}_{0}$, and $\hat{w}_{k}^{t} \equiv y_{k}^{t}, k=1, \ldots, d$ are the standard independent Wiener processes $w_{k}^{t}$ represented as the operators $\hat{w}_{k}^{t}=A_{-}^{k}(t)+A_{k}^{+}(t)$ on the Fock vacuum vector $\delta_{\varnothing} \in \mathcal{F}_{0}$ such that $w_{k}^{t} \simeq \hat{w}_{k}^{t} \delta_{o}$ (See the notations and more about the quantum stochastic calculus in Fock space in the Appendix 3). This model coincides with the signal plus noise model given above if

$$
\hat{e}^{k}(t)=\frac{1}{2}\left(a_{k}^{+}+a_{-}^{k}\right)(t)=\frac{1}{2 \lambda_{k}} \frac{\mathrm{d} w_{k}^{t}}{\mathrm{~d} t},
$$

where $a_{k}^{+}(t), a_{-}^{k}(t)$ are the canonical bosonic creation and annihilation field operators,

$$
\left[a_{k}^{+}(s), a_{l}^{+}(t)\right]=0,\left[a_{-}^{k}(s), a_{l}^{+}(t)\right]=\delta_{l}^{k} \delta(t-s),\left[a_{-}^{k}(s), a_{-}^{l}(t)\right]=0,
$$

defined as the generalized derivatives of the standard quantum Brownian motions $A_{k}^{+}(t)$ and $A_{-}^{k}(t)$ in Fock space $\mathcal{F}_{0}$. It was proved in [7, 86] that $Y_{k}^{t}$ is a commutative nondemolition process with respect to the system Heisenberg coordinate and momentum $P(t)=W(t)^{\dagger}(\mathrm{P} \otimes I) W(t)$ processes if they are perturbed by independent Langevin forces $f_{k}(t)$ of intensity $\tau_{k}^{2}=\left(\lambda_{k} \hbar\right)^{2}$, the generalized derivatives of $f_{k}^{t} \simeq \hat{f}_{k}^{t} \delta_{\varnothing}$ times $\lambda_{k}$, where $\hat{f}_{k}^{t}=i \hbar\left(A_{-}^{k}-A_{k}^{+}\right)(t)$ :

$$
\mathrm{d} P_{k}(t)+\phi_{k}^{\prime}(Q(t)) \mathrm{d} t=\lambda_{k} \mathrm{~d} \hat{f}_{k}^{t}, \quad P_{k}(t)=m \frac{\mathrm{d}}{\mathrm{d} t} Q^{k}(t) .
$$

Note that the quantum error operators $\hat{w}_{k}^{t}$ commute, but they do not commute with the perturbing quantum force operators $\hat{f}_{k}^{t}$ in Fock space due to the multiplication table

$$
\left(\mathrm{d} \hat{w}_{k}\right)^{2}=I \mathrm{~d} t, \quad \mathrm{~d} \hat{f}_{k} \mathrm{~d} \hat{w}_{l}=i \hbar I \delta_{l}^{k} \mathrm{~d} t, \quad \mathrm{~d} \hat{w}_{k} \mathrm{~d} \hat{f}_{l}=-i \hbar I \delta_{l}^{\kappa} \mathrm{d} t, \quad\left(\mathrm{~d} \hat{f}_{k}\right)^{2}=\hbar^{2} I \mathrm{~d} t .
$$

This corresponds to the canonical commutation relations for the renormalized derivatives $\hat{w}_{k}(t)$ and $\hat{f}_{l}(t)$, so that the true Heisenberg principle is fulfilled at the boundary $\sigma_{k} \tau_{k}=\hbar / 2$. Thus our quantum stochastic model of nondemolition observation is the minimal perturbation model for the given accuracy $\lambda$ of the continual indirect measurement of the position operators $Q(t)$ (the perturbation vanishes when $\lambda=0$ ).

3.3.2. Quantum state diffusion and filtering. Let us introduce the quantum stochastic wave equation for the unitary transformation $\Psi_{0}(t)=W(t) \Psi_{0}$ inducing Heisenberg dynamics which is decrepid by the quantum Langevin equation (3.22 
with white noise perturbation in terms. This equation is well understood in terms of the generalized derivatives

$$
\hat{f}_{k}(t)=\lambda_{k} \frac{\hbar}{i}\left(a_{k}^{+}-a_{-}^{k}\right)(t)=\lambda_{k} \frac{\mathrm{d} \hat{f}_{k}^{t}}{\mathrm{~d} t}
$$

of the standard quantum Brownian motions $A_{k}^{+}(t)$ and $A_{-}^{k}(t)$ defined by the commutation relations

$$
\left[A_{k}^{+}(s), A_{l}^{+}(t)\right]=0,\left[A_{-}^{k}(s), A_{l}^{+}(t)\right]=(t \wedge s) \delta_{l}^{k},\left[A_{-}^{k}(s), A_{-}^{l}(t)\right]=0
$$

in Fock space $\mathcal{F}_{0}(t \wedge s=\min \{s, t\})$ The corresponding quantum stochastic differential equation for the probability amplitude in $\mathfrak{h} \otimes \mathcal{F}_{0}$ is a particular case

$$
\mathrm{L}_{k}^{-\dagger}=-\mathrm{L}_{+}^{k}, \quad \mathrm{~L}_{+}^{k}=(\lambda \mathrm{Q})^{k} \equiv \mathrm{L}^{k}
$$

of the general quantum diffusion wave equation

$$
\mathrm{d} \Psi_{0}(t)+(\mathrm{K} \otimes I) \Psi_{0}(t) \mathrm{d} t=\left(\mathrm{L}_{+}^{k} \otimes \mathrm{d} A_{k}^{+}+\mathrm{L}_{k}^{-} \otimes \mathrm{d} A_{-}^{k}\right)(t) \Psi_{0}(t)
$$

which describes the unitary evolution in $\mathfrak{h} \otimes \mathcal{F}_{0}$ if $\mathrm{K}=\frac{i}{\hbar} \mathrm{H}-\frac{1}{2} \mathrm{~L}_{k}^{-} \mathrm{L}_{+}^{k}$, where $\mathrm{H}^{\dagger}=\mathrm{H}$ is the evolution Hamiltonian for the system in $\mathfrak{h}$. Using the quantum Itô formula, see the Appendix 3, it was proven in [7, 86] that it is equivalent to the Langevin equation

$$
\begin{aligned}
\mathrm{d} X(t)= & \left(f\left(X L+L^{\dagger} X\right)+L^{\dagger} X L-K^{\dagger} X-X K\right)(t) \mathrm{d} t \\
& +\left(f X+L^{\dagger} X-X L\right)(t) \mathrm{d} A_{-}+\left(f X+X L-L^{\dagger} X\right)(t) \mathrm{d} A^{+}
\end{aligned}
$$

for any quantum stochastic Heisenberg process

$$
X(t, f)=W(t)^{\dagger}\left(\mathrm{X} \otimes \exp \left[\int_{0}^{t}\left(f^{k}(r) \mathrm{d} \hat{w}_{k}^{r}-\frac{1}{2} f(r)^{2} \mathrm{~d} r\right)\right]\right) W(t),
$$

where $f^{k}(t)$ are a test function for the output process $w_{k}^{t}$ and

$$
K(t)=W(t)^{\dagger}(\mathrm{K} \otimes \mathrm{I}) W(t), \quad L^{k}(t)=W(t)^{\dagger}\left(\mathrm{L}^{k} \otimes \mathrm{I}\right) W(t) .
$$

The Langevin equation (3.22) for the system coordinate $X(t)=W(t)^{\dagger}(\mathrm{Q} \otimes I) W(t)$ and for the output processes $Y(t, f)$ corresponding to $\mathrm{X}=\mathrm{I}$ follow straightforward in the case $\mathrm{L}=\lambda \mathrm{Q}, \mathrm{H}=\mathrm{P}^{2} / 2 m+\phi(\mathrm{Q})$.

In the next section we shall show that this unitary evolution is the interaction picture for a unitary group evolution $U^{t}$ corresponding to a Dirac type boundary value problem for a generalized Schrödinger equation in an extended product Hilbert space $\mathfrak{h} \otimes \mathcal{G}$. Here we prove that the quantum stochastic evolution (3.23) in $\mathfrak{h} \otimes \mathcal{F}_{0}$ coincides with the quantum state diffusion in $\mathfrak{h}$ if it is considered only for the initial product states $\psi \otimes \delta_{\varnothing}$ with $\delta_{\varnothing}$ being the Fock vacuum state vector in $\mathcal{F}_{0}$.

Quantum state diffusion is a nonlinear, nonunitary, irreversible stochastic form of quantum mechanics with trajectories put forward by Gisin and Percival 40, 41] in the early 90's as a new, primary quantum theory which includes the diffusive reduction process into the wave equation for pure quantum states. It has been criticized, quite rightly, as an incomplete theory which does not satisfy the linear superposition principle for the waves, and for not explaining the origin of irreversible dissipativity which is build into the equation 'by hands'. In fact the 'primary' equation had been derived even earlier as the posterior state diffusion equation for pure states $\psi_{\omega}=\psi(\omega) /\|\psi(\omega)\|$ from the linear unitary quantum diffusion equation (3.23) by the following method as a particular type of the general quantum filtering 
equation in [7, 89, 94]. Here we shall show only how to derive the corresponding stochastic linear decoherence equation $\{3.11$ for $\psi(t, w)=V(t, w) \psi$ when all the independent increment processes $y_{k}^{t}$ are of the diffusive type $y_{k}^{t}=w_{k}^{t}$ :

$$
\mathrm{d} \psi(t, \omega)+\mathrm{K} \psi(t, \omega) \mathrm{d} t=\mathrm{L}^{k} \psi(t, \omega) \mathrm{d} w_{k}^{t}, \quad \psi(0)=\psi
$$

Note that the resolving stochastic propagator $V(t, \omega)$ for this equation defines the isometries

$$
V(t)^{\dagger} V(t)=\int V(t, \omega)^{\dagger} V(t, \omega) \mathrm{d} \mu=1
$$

of the system Hilbert space $\mathfrak{h}$ into the Wiener Hilbert space $L_{\mu}^{2}$ of square integrable functionals of the diffusive trajectories $\omega=\{w(t)\}$ with respect to the standard Gaussian measure $\mu=\mathrm{P}_{w}$ if $\mathrm{K}+\mathrm{K}^{\dagger}=\mathrm{L}^{\dagger} \mathrm{L}$.

Let us represent these Wiener processes in the equation by operators $\hat{w}_{k}^{t}=$ $A_{k}^{+}+A_{-}^{k}$ on the Fock space vacuum $\delta_{\varnothing}$ using the unitary equivalence $w_{k}^{t} \simeq \hat{w}_{k}^{t} \delta_{\varnothing}$ in the notation explained in the Appendix 3. Then the corresponding operator equation

$$
\mathrm{d} \hat{\psi}(t)+\mathrm{K} \hat{\psi}(t) \mathrm{d} t=\left(\mathrm{L}^{k} \mathrm{~d} A_{k}^{+}+\mathrm{L}_{k}^{\dagger} \mathrm{d} A_{-}^{k}\right) \hat{\psi}(t), \quad \hat{\psi}(0)=\psi \otimes \delta_{\varnothing}, \psi \in \mathfrak{h},
$$

with $\mathrm{L}_{k}^{\dagger}=\lambda \mathrm{Q}^{k}=\mathrm{L}^{k}$, coincides with the quantum diffusion Schrödinger equation (3.23), where $\mathrm{L}_{+}^{k}=\mathrm{L}^{k}, \mathrm{~L}_{k}^{-}=-\mathrm{L}_{k}$ on the same initial product-states $\Psi_{0}(0)=$ $\psi \otimes \delta_{\varnothing}$. Indeed, as it was noted in 86 , due to the adaptedness

$$
\hat{\psi}(t)=\hat{\psi}^{t} \otimes \delta_{\varnothing}, \quad \Psi_{0}(t)=\Psi_{0}^{t} \otimes \delta_{\varnothing}
$$

both right had sides of these equations coincide on future vacuum $\delta_{\varnothing}$ if $\hat{\psi}^{t}=\Psi_{0}^{t}$ as

$$
\begin{aligned}
\mathrm{L}^{k} \mathrm{~d} \hat{w}_{k}^{t} \hat{\psi}(t) & =\left(\mathrm{L}^{k} \mathrm{~d} A_{k}^{+}+\mathrm{L}_{k}^{\dagger} \mathrm{d} A_{-}^{k}\right)\left(\hat{\psi}^{t} \otimes \delta_{\varnothing}\right)=\mathrm{L}^{k} \hat{\psi}^{t} \otimes \mathrm{d} A_{k}^{+} \delta_{\varnothing} \\
\frac{i}{\hbar} \mathrm{L}^{k} \mathrm{~d} \hat{f}_{k}^{t} \Psi_{0}(t) & =\left(\mathrm{L}^{k} \mathrm{~d} A_{k}^{+}-\mathrm{L}_{k} \mathrm{~d} A_{-}^{k}\right)\left(\Psi_{0}^{t} \otimes \delta_{\varnothing}\right)=\mathrm{L}^{k} \Psi_{0}^{t} \otimes \mathrm{d} A_{k}^{+} \delta_{\varnothing}
\end{aligned}
$$

(the annihilation processes $A_{-}^{k}$ are zero on the vacuum $\delta_{\varnothing}$ ). By virtue of the coincidence of the initial data $\hat{\psi}^{0}=\psi=\Psi_{0}^{t}$ this proves that $\hat{\psi}(t)=\Psi_{0}(t)$ for all $t>0$. Note that the quantum stochastic evolutions $\hat{\psi}(t)$ and $\Psi_{0}(t)$, when extended on the whole space $\mathfrak{h} \otimes \mathcal{G}_{0}$, are described by the different propagators $V(t)$ and $W(t)$ as $\hat{\psi}(t)=V(t) \hat{\psi}, \Psi_{0}(t)=W(t) \Psi_{0}$. The first one is unbounded and even not well defined on the whole space $\mathfrak{h} \otimes \mathcal{G}$, while the second one is unitary, resolving another stochastic differential equation

$$
\mathrm{d} \psi_{0}(t)+\left(\frac{i}{\hbar} \mathrm{H}+\frac{1}{2} \mathrm{Q}^{k} \lambda_{k}^{2} \mathrm{Q}^{k}\right) \psi_{0}(t) \mathrm{d} t=\frac{i}{\hbar} \lambda \mathrm{Q}^{k} \psi_{0}(t) \mathrm{d} f_{k}^{t}, \quad \psi_{0}(0)=\psi .
$$

by the unitary propagator $\mathrm{W}(t, f)=W(t)\left(\mathrm{I} \otimes \delta_{\varnothing}\right)$ for each $f$ in $\mathfrak{h}$ as the stochastic function $\psi_{0}(t, f)=\mathrm{W}(t, f) \psi$ on another classical probability space.

Thus the stochastic decoherence equation

$$
\mathrm{d} \psi(t)+\left(\frac{i}{\hbar} \mathrm{H}+\frac{1}{2} \mathrm{Q}^{k} \lambda_{k}^{2} \mathrm{Q}^{k}\right) \psi(t) \mathrm{d} t=\lambda_{k} \mathrm{Q}^{k} \psi(t) \mathrm{d} w_{k}^{t}, \quad \psi(0)=\psi
$$

for the continuous observation of the position of a quantum particle with $\mathrm{H}=$ $\frac{1}{2 m} \mathrm{P}^{2}+\phi(\mathrm{Q})$ was derived for the unitary quantum stochastic evolution as an example of the general decoherence equation (3.11) which was obtained in this way in [7]. It was explicitly solved in [7, 89, 94] for the case of linear and quadratic 
potentials $\phi$, and it was shown that this solution coincides with the optimal quantum linear filtering solution obtained earlier in [4, 5] if the initial wave packet is Gaussian.

The nonlinear stochastic posterior equation for this particular case was derived independently by Diosi [8] and (as an example) in [7, 89]. It has the following form

$$
\mathrm{d} \psi_{w}(t)+\left(\frac{i}{\hbar} \mathrm{H}+\frac{1}{2} \widetilde{\mathrm{Q}}^{k}(t) \lambda_{k}^{2} \widetilde{\mathrm{Q}}^{k}(t)\right) \psi_{w}(t) \mathrm{d} t=\lambda_{k} \widetilde{\mathrm{Q}}^{k}(t) \psi_{w}(t) \mathrm{d} \tilde{w}_{k}^{t},
$$

where $\widetilde{\mathrm{Q}}(t)=\mathrm{Q}-\hat{q}(t)$ with $\hat{q}^{k}(t)$ defined as the multiplication operators by the components $q^{k}(t, w)=\psi_{w}^{\dagger}(t) \mathrm{Q}^{k}(t) \psi_{w}(t)$ of the posterior expectation (statistical prediction) of the coordinate $\mathrm{Q}$, and

$$
\mathrm{d} \tilde{w}_{k}^{t}=\mathrm{d} w_{k}^{t}-2 \lambda_{k} \hat{q}^{k}(t) \mathrm{d} t=\mathrm{d} y_{k}^{t}-\hat{x}_{k}(t) \mathrm{d} t, \quad \hat{x}_{k}(t)=2(\lambda \hat{q})^{k}(t) .
$$

Note that the innovating output processes $\tilde{w}_{k}^{t}$ are also standard Wiener processes with respect to the output probability measure $\mathrm{d} \tilde{\mu}=\lim \operatorname{Pr}(t, \mathrm{~d} \omega)$, but not with respect to the Wiener probability measure $\mu=\operatorname{Pr}(0, \mathrm{~d} \omega)$ for the input noise $w_{k}^{t}$.

Let us give the explicit solution of this stochastic wave equation for the free particle $(\phi=0)$ in one dimension and the stationary Gaussian initial wave packet which was found in $[7,89,94]$. One can show [95, 96 that the nondemolition observation of such particle is described by filtering of quantum noise which results in the continual collapse of any wave packet to the Gaussian stationary one centered at the posterior expectation $q(t, w)$ with finite dispersion $\left\|(\hat{q}(t)-\mathrm{Q}) \psi_{\omega}(t)\right\|^{2} \rightarrow$ $2 \lambda(\hbar / m)^{1 / 2}$. This center can be found from the linear Newton equation

$$
\frac{\mathrm{d}^{2}}{\mathrm{~d} t^{2}} z(t)+2 \kappa \frac{\mathrm{d}}{\mathrm{d} t} z(t)+2 \kappa^{2} z(t)=-g(t),
$$

for the deviation process $z(t)=q(t)-x(t)$, where $x(t)$ is an expected trajectory of the output process (3.21) with $z(0)=q_{0}-x(0), z^{\prime}(0)=v_{0}-x^{\prime}(0)$. Here $\kappa=\lambda(\hbar / m)^{1 / 2}$ is the decay rate which is also the frequency of effective oscillations, $q_{0}=\langle\hat{x}\rangle, v_{0}=\langle\hat{p} / m\rangle$ are the initial expectations and $g(t)=x^{\prime \prime}(t)$ is the effective gravitation for the particle in the moving framework of $x(t)$. The following figure illustrate the continuous collapse $z(t) \rightarrow 0$ of the posterior trajectory $q(t)$ towards a linear trajectory $x(t)$.

The posterior position expectation $q(t)$ in the absence of effective gravitation, $x^{\prime \prime}(t)=0$, for the linear trajectory $x(t)=u t-q$ collapses to the expected input trajectory $x(t)$ with the rate $\kappa=\lambda(\hbar / m)^{1 / 2}$, remaining not collapsed, $q_{0}(t)=v_{0} t$ 
in the framework where $q_{0}=0$, only in the classical limit $\hbar / m \rightarrow 0$ or absence of observation $\lambda=0$. This is the graph of

$$
q_{0}(t)=v_{0} t, \quad q(t)=u t+e^{-\kappa t}\left(q \cos \kappa t+\left(q+\kappa^{-1}\left(v_{0}-u\right)\right) \sin \kappa t\right)-q
$$

obtained as $q(t)=x(t)+z(t)$ by explicit solving of the second order linear equation for $z(t)$.

3.3.3. The dynamical boundary-value realization. Finally, let us set up an explicitly solvable microscopic problem underlying all quantum diffusion and more general quantum noise Langevin models. We shall see that all such models exactly correspond to Dirac type boundary value problems for a Poisson flow of independent quantum particles interacting with the quantum system under the observation at the boundary $r=0$ of the half line $\mathbb{R}_{+}$in an additional dimension, exactly as it was done for the quantum jumps in (3.11). One can think that the coordinate $r>0$ of this extra dimension is a physical realization of localizable time, at least it is so for any free evolution Hamiltonian $\varepsilon(p)>0$ of the incoming quantum particles in the ultrarelativistic limit $\langle p\rangle \rightarrow-\infty$ such that the average velocity in an initial state is a finite constant, $c=\left\langle\varepsilon^{\prime}(p)\right\rangle \rightarrow 1$ say. Thus we are going to solve the problem of quantum trajectories, individual decoherence, state diffusion, or permanent reduction problem as the following time continuous quantum measurement problem which we already formulated and solved in the Sec. 5.3 for the time discrete case:

Given a reduction family $V(t, \omega)=V\left(t, w_{0}^{t]}\right), t \in \mathbb{R}_{+}$of isometries on $\mathfrak{h}$ into $\mathfrak{h} \otimes L_{\mu}^{2}$ resolving the state diffusion equation 3.25) with respect to the input probability measure $\mu=\mathrm{P}_{w}$ for the standard Wiener noises $w_{k}^{t}$, find a triple $(\mathcal{G}, \mathfrak{A}, \Phi)$ consisting of a Hilbert space $\mathcal{G}=\mathcal{G}_{-} \otimes \mathcal{G}_{+}$embedding the Wiener Hilbert space $L_{\mu}^{2}$ by an isometry into $\mathcal{G}_{+}$, an algebra $\mathfrak{A}=\mathfrak{A}_{-} \otimes \mathfrak{A}_{+}$on $\mathcal{G}$ with an Abelian subalgebra $\mathfrak{A}_{-}$generated by a compatible continuous family $Y_{-\infty}^{0]}=\left\{Y_{k}^{s}, k=1, \ldots, d, s \leq 0\right\}$ of observables on $\mathcal{G}_{-}$, and a state-vector $\Phi^{\circ}=\Phi_{-}^{\circ} \otimes \Phi_{+}^{\circ} \in \mathcal{G}$ such that there exist a time continuous unitary group $U^{t}$ on $\mathcal{H}=\mathfrak{h} \otimes \mathcal{G}$ inducing a semigroup of endomorphisms $\mathfrak{B} \ni B \mapsto U^{-t} B U^{t} \in \mathfrak{B}$ on the product algebra $\mathfrak{B}=\mathcal{B}(\mathfrak{h}) \otimes \mathfrak{A}$, with

$$
\begin{aligned}
\pi^{t}\left(\hat{g}_{-t} \otimes \mathrm{B}\right) & :=\left(\mathrm{I} \otimes \Phi^{\circ}\right)^{\dagger} U^{-t}\left(\mathrm{~B} \otimes g_{-t}\left(Y_{-t}^{0]}\right)\right) U^{t}\left(\mathrm{I} \otimes \Phi^{\circ}\right) \\
= & \int g\left(w_{0}^{t]}\right) V\left(t, w_{0}^{t]}\right)^{\dagger} \mathrm{B} V\left(t, w_{0}^{t]}\right) \mathrm{dP}_{w} \equiv \mathrm{M}\left[g V(t)^{\dagger} \mathrm{B} V(t)\right]
\end{aligned}
$$

for any $\mathrm{B} \in \mathcal{B}(\mathfrak{h})$ and any operator $\hat{g}_{-t}=\hat{g}_{-t}\left(Y_{-t}^{0]}\right) \in \mathfrak{C}$ represented as the shifted functional $\hat{g}_{-t}\left(y_{-t}^{0]}\right)=g\left(y_{0}^{t]}\right)$ of $Y_{-t}^{-0]}=\left\{Y^{s}: s \in(-t, 0]\right\}$ on $\mathcal{G}$ by any measurable functional $g$ of $y_{0}^{t]}=\left\{y^{r}: r \in(0, t]\right\}$ with arbitrary $t>0$.

We have already dilated the state diffusion equation (3.25) to a quantum stochastic unitary evolution $W(t)$ resolving the quantum stochastic Schrödinger equation (3.23) on the system Hilbert $\mathfrak{h}$ tensored the Fock space $\mathcal{F}_{0}$ such that $W(t)\left(\mathrm{I} \otimes \delta_{\varnothing}\right)=$ $V(t)$, where $\delta_{\varnothing} \in \mathcal{F}_{0}$ is the Fock vacuum vector. In fact the state diffusion equation was first derived [7, 89] in this way from even more general quantum stochastic unitary evolution which satisfy the equation

$$
\left(\mathrm{I} \otimes \delta_{\varnothing}\right)^{\dagger} W(t)^{\dagger}\left(\mathrm{B} \otimes g\left(\hat{w}_{0}^{t]}\right)\right) W(t)\left(\mathrm{I} \otimes \delta_{\varnothing}\right)=\mathrm{M}\left[g V(t)^{\dagger} \mathrm{B} V(t)\right] .
$$


Indeed, this equation is satisfied for the model (3.23) as one can easily check for

$$
g\left(w_{0}^{t]}\right)=\exp \left[\int_{0}^{t}\left(f^{k}(r) \mathrm{d} w_{k}^{r}-\frac{1}{2} f(r)^{2} \mathrm{~d} r\right)\right]
$$

given by a test vector function $f$ by conditioning the Langevin equation (3.24) with respect to the vacuum vector $\delta_{\varnothing}$ :

$$
\left(\mathrm{I} \otimes \delta_{\varnothing}\right)^{\dagger}\left(\mathrm{d} X+\left(K^{\dagger} X+X K-L^{\dagger} X L-\left(X L+L^{\dagger} X\right) f\right) \mathrm{d} t\right)\left(\mathrm{I} \otimes \delta_{\varnothing}\right)=0 .
$$

Obviously this equation coincides with the conditional expectation

$$
\mathrm{M}\left[\mathrm{d} B(t)+\left(\mathrm{K}^{\dagger} B(t)+B(t) \mathrm{K}-\mathrm{L}^{\dagger} B(t) \mathrm{L}-\left(B(t) L+L^{\dagger} B(t)\right) f(t)\right) \mathrm{d} t\right]=0
$$

for the stochastic process $B(t)=V(t)^{\dagger} g \mathrm{X} V(t)$ which satisfies the stochastic Itô equation

$\mathrm{d} B+g V^{\dagger}\left(\mathrm{K}^{\dagger} \mathrm{X}+\mathrm{XK}-\mathrm{L}^{\dagger} \mathrm{XL}-\left(\mathrm{XL}+\mathrm{L}^{\dagger} \mathrm{X}\right) f\right) V \mathrm{~d} t=g V^{\dagger}\left(\mathrm{L}^{\dagger} \mathrm{X}+\mathrm{XL}+\mathrm{X}\right) V \mathrm{~d} w^{t}$.

This however doesn't give yet the complete solution of the quantum measurement problem as formulated above because the algebra $\mathfrak{B}_{0}$ generated by $\mathrm{B} \otimes I$ and the Langevin forces $\hat{f}_{k}^{t}$ does not contain the measurement processes $\hat{w}_{k}^{t}$ which do not commute with $\hat{f}_{k}^{t}$, and the unitary family $W(t)$ does not form unitary group but only cocycle

$$
T_{t} W(s) T_{-t} W(t)=W(s+t), \quad \forall s, t>0
$$

with respect to the isometric but not unitary right shift semigroup $T_{t}$ in $\mathcal{F}_{0}$.

Let $T_{t}$ be the one parametric continuous unitary shift group on $\mathcal{F}^{0]} \otimes \mathcal{F}_{0}$ extending the defining from $\mathcal{F}_{0}$. It describes the free evolution by right shifts $\Phi_{t}(\omega)=$ $\Phi(\omega-t)$ in Fock space over the whole line $\mathbb{R}$ (Here and below we use the notations from the Sec. 6.3). Then one can easily find the unitary group

$$
U^{t}=T_{-t}\left(I^{0} \otimes \mathrm{I} \otimes W(t)\right) T_{t}
$$

on $\mathcal{F}_{0} \otimes \mathfrak{h} \otimes \mathcal{F}^{0]}$ inducing the quantum stochastic evolution as the interaction representation $U(t)=T_{t} U^{t}$ on the Hilbert space $\mathfrak{h} \otimes \mathcal{G}_{0}$. In fact this evolution corresponds to an unphysical coordinate discontinuity problem at the origin $s=0$ which is not invariant under the reflection of time $t \mapsto-t$. Instead, we shall formulate the unitary equivalent boundary value problem in the Poisson space $\mathcal{G}=\mathcal{G}_{-} \otimes \mathcal{G}_{+}$for two semi-infinite strings on $\mathbb{R}_{+}$, one is the living place for the quantum noise generated by a Poisson flow of incoming waves of quantum particles of the intensity $\nu>0$, and the other one is for the outgoing classical particles carrying the information after a unitary interaction with the measured quantum system at the origin $r=0$. The probability amplitudes $\Phi \in \mathcal{G}$ are represented by the $\mathbb{G}^{\otimes}=\mathfrak{g}_{-}^{\otimes} \otimes \mathfrak{g}_{+}^{\otimes}$-valued functions $\Phi\left(v_{-}, v_{+}\right)$of two infinite sequences $v_{ \pm}=\left\{ \pm r_{1}, \pm r_{2}, \ldots\right\} \subset \mathbb{R}_{+}$of the coordinates of the particles in the increasing order $r_{1}<r_{2}<\ldots$ such that

$$
\|\Phi\|^{2}=\iint\left\|\Phi\left(v_{-}, v_{+}\right)\right\|^{2} \mathrm{P}_{\nu}\left(\mathrm{d} v_{-}\right) \mathrm{P}_{\nu}\left(\mathrm{d} v_{+}\right)<\infty
$$

with respect to the product of two copies of the Poisson probability measure $\mathrm{P}_{\nu}$ defined by the constant intensity $\nu>0$ on $\mathbb{R}_{+}$. Here $\mathfrak{g}^{\otimes}$ is the infinite tensor product of $\mathfrak{g}=\mathbb{C}^{d}$ obtained by the completion of the linear span of $\chi_{1} \otimes \chi_{2} \otimes \ldots$ with almost all multipliers $\chi_{n}=\varphi$ given by a unit vector $\varphi \in \mathbb{C}^{d}$ such that the 
infinite product $\|\Phi(v)\|=\prod_{r \in v}\|f(r)\|$ for $\Phi(v)=\otimes_{r \in v} f(r)$ with $f\left(r_{n}\right)=\chi_{n}$ is well defined as it has all but finite number of multipliers $\left\|\chi_{n}\right\|$ equal 1 . The unitary transformation $\digamma \mapsto \Phi$ from Fock space $\mathcal{F} \ni \digamma$ to the corresponding Poisson one $\mathcal{G}$ can be written as

$$
\Phi=\lim _{t \rightarrow \infty} \mathrm{e}^{\varphi^{i} A_{i}^{+}(t)} \mathrm{e}^{-\varphi_{k} A_{-}^{k}(t)} \nu^{-\frac{1}{2} A_{i}^{i}(t)} \digamma \equiv I_{\nu}(\varphi) \digamma,
$$

where $\varphi_{k}=\nu \bar{\varphi}^{k}$ for the Poisson intensity $\nu>0$ and the unit vector $\varphi=\left(\varphi^{i}\right)$ defined by the initial probability amplitude $\varphi \in \mathfrak{g}$ for the auxiliary particles to be in a state $k=1, \ldots, d$. Here $A_{\iota}^{\kappa}(t)$ are the QS integrators defined in Appedix 3, and the limit is taken on the dense subspace $\cup_{t>0} \mathcal{F}_{0}^{t]}$ of vacuum-adapted Fock functions $\digamma_{t} \in \mathcal{F}_{0}$ and extended then onto $\mathcal{F}_{0}$ by easily proved isometry $\left\|\digamma_{t}\right\|=\left\|\Phi_{t}\right\|$ for $\Phi_{t}=I_{\nu}(\varphi) \digamma_{t}$.

The free evolution in $\mathcal{G}$ is the same as in the Section 6.3,

$$
T_{t} \Phi\left(v_{-}, v_{+}\right)=\Phi\left(v_{-}^{t}, v_{+}^{t}\right),
$$

where $v_{ \pm}^{t}= \pm\left[\left(\left[\left(-v_{-}\right) \cup\left(+v_{+}\right)\right]-t\right) \cap \mathbb{R}_{ \pm}\right]$. It is given by the second quantization (3.16) of the Dirac Hamiltonian in one dimension on $\mathbb{R}_{+}$.

In order to formulate the boundary value problem in the space $\mathcal{H}=\mathfrak{h} \otimes \mathcal{G}$ corresponding to the quantum stochastic equations of the diffusive type (3.23) let us introduce the notation

$$
\Phi\left(0^{k} \sqcup v_{ \pm}\right)=\lim _{r \searrow 0}\left(\langle k| \otimes \mathrm{I}_{1} \otimes \mathrm{I}_{2} \ldots\right) \Phi\left( \pm r, \pm r_{1}, \pm r_{2}, \ldots\right),
$$

where $\langle k|=d^{-1 / 2}\left(\delta_{1}^{k}, \ldots, \delta_{l}^{k}\right)$ acts as the unit bra-vector evaluating the $k$-th projection of the state vector $\Phi\left( \pm r \sqcup v_{ \pm}\right)$with $r<r_{1}<r_{2}<\ldots$ corresponding to the nearest to the boundary $r=0$ particle in one of the strings on $\mathbb{R}_{+}$.

The unitary group evolution $U^{t}$ corresponding to the scattering interaction at the boundary with the continuously measured system which has its own free evolution described by the energy operator $\mathrm{E}=\mathrm{E}^{\dagger}$ can be obtained by resolving the following generalized Schrödinger equation

$$
\frac{\partial}{\partial t} \Psi^{t}\left(v_{-}, v_{+}\right)-\frac{\mathrm{i}}{\hbar} P \Psi^{t}\left(t, v_{-}, v_{+}\right)=\mathrm{G}_{k}^{-} \Psi^{t}\left(v_{-}, 0^{k} \sqcup v_{+}\right)+\mathrm{G}_{+}^{-} \Psi^{t}\left(v_{-}, v_{+}\right)
$$

with the Dirac zero current boundary condition at the origin $r=0$

$$
\Psi^{t}\left(0^{i} \sqcup v_{-}, v_{+}\right)=\mathrm{G}_{+}^{i} \Psi^{t}\left(v_{-}, v_{+}\right)+\mathrm{G}_{k}^{i} \Psi^{t}\left(v_{-}, 0^{k} \sqcup v_{+}\right), \quad \forall t>0, v_{ \pm}>0 .
$$

Here $\mathrm{G}=\left[\mathrm{G}_{k}^{i}\right]$ is unitary, $\mathrm{G}^{-1}=\mathrm{G}^{\dagger}$, like the scattering operator $\mathrm{S}$ in the simpler quantum jump boundary value problem (3.18), and the other system operators $\mathrm{G}_{\iota}^{\kappa}$, with $\iota=-, i$ and $\kappa=k,+$ for any $i, k=1, \ldots, d$ are chosen as

$$
\mathrm{G}^{-}+\nu \mathrm{G}_{+}^{\dagger} \mathrm{G}=\mathrm{O}, \quad \mathrm{G}_{+}^{-}+\frac{\nu}{2} \mathrm{G}_{+}^{\dagger} \mathrm{G}_{+}+\frac{i}{\hbar} \mathrm{E}=\mathrm{O}
$$


Note that these conditions can be written as pseudo-unitarity of the following triangular block-matrix

$$
\left[\begin{array}{lll}
\mathrm{I} & \mathrm{G}^{-} & \mathrm{G}_{+}^{-} \\
\mathrm{O} & \mathrm{G} & \mathrm{G}_{+} \\
\mathrm{O} & \mathrm{O} & \mathrm{I}
\end{array}\right]^{-1}=\left[\begin{array}{ccc}
\mathrm{O} & \mathrm{O} & \mathrm{I} \\
\mathrm{O} & \nu \mathrm{I} & \mathrm{O} \\
\mathrm{I} & \mathrm{O} & \mathrm{O}
\end{array}\right]^{-1}\left[\begin{array}{lll}
\mathrm{I} & \mathrm{G}^{-} & \mathrm{G}_{+}^{-} \\
\mathrm{O} & \mathrm{G} & \mathrm{G}_{+} \\
\mathrm{O} & \mathrm{O} & \mathrm{I}
\end{array}\right]^{\dagger}\left[\begin{array}{ccc}
\mathrm{O} & \mathrm{O} & \mathrm{I} \\
\mathrm{O} & \nu \mathrm{I} & \mathrm{O} \\
1 & \mathrm{O} & \mathrm{O}
\end{array}\right]
$$

As it was proved in 79, 83 this is necessary (and sufficient at if all operators are bounded) condition for the unitarity $W(t)^{-1}=W(t)^{\dagger}$ of the cocycle solution resolving the quantum stochastic differential equation

$$
\mathrm{d} \Psi_{0}(t)=\left(\mathrm{G}_{\kappa}^{\iota}-\delta_{\kappa}^{\iota} \mathrm{I}\right) \Psi_{0}(t) \mathrm{d} A_{\iota}^{\kappa}, \quad \Psi_{0}(0)=\Psi_{0}
$$

in the Hilbert space $\mathcal{H}_{0}=\mathfrak{h} \otimes \mathcal{G}_{0}$ where $\mathcal{G}_{0}$ is identified with the space $\mathcal{G}_{+}=\mathbb{G}^{\otimes} \otimes L_{\mu}^{2}$ for the Poisson measure $\mu=\mathrm{P}_{\nu}$ with the intensity $\nu$ on $\mathbb{R}_{+}$. This is the general form for the quantum stochastic equation (3.23) where $\mathrm{d} A_{-}^{+}=\mathrm{d} t$ in the Poisson space see the Appendix 3 for more detail explanations of these notations. Our recent results partially published in [99, 100, 101] prove that this quantum stochastic evolution extended as the identity $I_{-}$also on the component $\mathcal{G}_{-}$for the scattered particles, is nothing but the interaction representation $U^{t}=T_{-t}\left(I_{-} \otimes W(t)\right)$ for the unitary group $U^{t}$ resolving our boundary value problem in $\mathfrak{h} \otimes \mathbb{G}^{\otimes}$ times the Poisson space $L_{\mu}^{2}$. Thus the pseudounitarity condition (3.29) is necessary (and sufficient if the operators $\mathrm{G}_{\kappa}^{\iota}$ are bounded) for the self-adjointness of the Dirac type boundary value problem (3.27), (3.28).

The generators $\mathrm{G}_{\kappa}^{\iota}$ of this boundary value problem define the generators $\mathrm{S}_{\kappa}^{\iota}$ of the corresponding quantum stochastic equation in Fock space by the following transformation

$$
\begin{aligned}
& \mathrm{S}_{+}^{i}=\nu^{1 / 2}\left(\mathrm{G}_{+}^{i}+\mathrm{G}_{k}^{i} \varphi^{k}-\varphi^{i}\right), \mathrm{S}_{k}^{-}=\nu^{-1 / 2}\left(\mathrm{G}_{k}^{-}+\varphi_{i} \mathrm{G}_{k}^{i}-\varphi_{k}\right) \\
& \mathrm{S}_{+}^{-}=\mathrm{G}_{+}^{-}+\varphi_{i} \mathrm{G}_{+}^{i}+\mathrm{G}_{k}^{-} \varphi^{k}+\varphi_{i}\left(\mathrm{G}_{k}^{i}-\delta_{k}^{i} \mathrm{I}\right) \varphi^{k}, \quad \mathrm{~S}_{k}^{i}=\mathrm{G}_{k}^{i},
\end{aligned}
$$

induced by the canonical transformation $I_{\nu}(\varphi)$.

The quantum state diffusion equation (3.25) for the continuous measurement of the coordinates $\mathrm{Q}^{k}$ corresponds to the particular case 3.22 )of the quantum stochastic differential equation in Fock space, with

$$
\begin{aligned}
& \mathrm{S}_{+}^{i}=\nu^{1 / 2} \mathrm{G}_{+}^{i}, \quad \mathrm{~S}_{k}^{-}=\nu^{-1 / 2} \mathrm{G}_{k}^{-} \\
& \mathrm{S}_{+}^{-}=\mathrm{G}_{+}^{-}+\varphi_{i} \mathrm{G}_{+}^{i}+\mathrm{G}_{k}^{-} \varphi^{k}, \quad \mathrm{~S}_{k}^{i}=\delta_{k}^{i} \mathrm{I},
\end{aligned}
$$

and $\mathrm{G}_{+}^{i}=\mathrm{Q}^{i}, \mathrm{G}_{k}^{-}=\nu \mathrm{Q}^{k}$ such that all coupling constants $\lambda_{k}=\nu^{1 / 2}$. are equal to the square root of the flow intensity $\nu$. The operators $\mathrm{G}_{+}^{i}=\varphi^{i} \mathrm{Q}^{i}$ and $\mathrm{G}_{k}^{-}=\mathrm{Q}_{k} \varphi_{k}$ corresponding to the different couplings $\lambda_{k}$ can also be obtained from the purely jump model in the central limit $\nu \mapsto \infty$ as it was done in 85. In this case

$$
\mathrm{S}_{+}^{i}=\nu^{1 / 2}(\mathrm{G}-\mathrm{I})_{k}^{i} \varphi^{k} \rightarrow-\mathrm{i} \lambda \varphi^{i} \mathrm{Q}^{i},
$$

with $\varphi^{k}=\mathrm{i} \lambda_{k} / \lambda$.

And finally, we have to find the operator processes $Y_{k}^{s}, s \leq 0$ on the Hilbert space $\mathcal{G}_{-}$which reproduce the standard Wiener noises $w_{k}^{t}$ in the state diffusion when our dynamical model is conditioned (filtered) with respect their nondemolition measurement. As the candidates let us consider the field coordinate processes

$$
X_{k}^{-t}=A_{k}^{+}(-t, 0]+A_{-}^{k}(-t, 0]=T_{-t}\left(A_{k}^{+}(0, t]+A_{-}^{k}(0, t]\right) T_{t}
$$


which are given by the creation and annihilation processes $A^{+}(t)$ and $A_{-}(t)$ shifted from $\mathcal{G}_{+}$. In our Poisson space model of $\mathcal{G}$ they have not zero expectations

$$
\Phi^{\dagger} X_{k}^{-t} \Phi=\Phi^{\dagger}\left(A_{k}^{+}(0, t]+A_{-}^{k}(0, t]\right) \Phi=2 \nu^{1 / 2} t
$$

in the ground state $\Phi=I_{\nu}(\varphi) \delta_{\varnothing}$ corresponding to the vacuum vector $\delta_{\varnothing}$ in the Fock space. This state is given as the infinite tensor product $\Phi^{\circ}=\varphi_{-}^{\otimes} \otimes \varphi_{+}^{\otimes}$ of all equal probability amplitudes $\varphi_{-}=\varphi=\varphi_{+}$in $\mathfrak{g}=\mathbb{C}^{d}$ for each sequence $v_{-}$and $v_{+}$. Hence the independent increment processes $Y_{k}^{t}=T_{t} Y_{k}^{-t} T_{-t}$ corresponding to the standard Wiener noises $w_{k}^{t}$ represented in Fock spaces as $\hat{w}_{k}^{t}=A_{k}^{+}(t)+A_{-}^{k}(t)$ are the compensated processes $Y_{k}^{-t}=X_{k}^{-t}-2 \nu^{1 / 2} t$. This unitary equivalence of $Y_{k}^{t}$ and $\hat{w}_{k}^{t}$ under the Fock-Poisson transformation $I_{\nu}(\varphi)$, and the deduction given above of the quantum state diffusion from the quantum stochastic signal plus noise model (3.21) for continuous observation in Fock space, completes the solution of the quantum measurement model in its rigorous formulation.

\section{Conclusion: A quantum message from the Future}

Although the conventional formulation of quantum mechanics and quantum field theory is inadequate for the temporal treatment of modern experiments with the individual quantum system in real time, it has been shown that the latest developments in quantum probability, stochastics and in quantum information theory made it possible to reconcile the dynamical and statistical aspects of its interpretation. All such phenomena as quantum events, causality, decoherence, quantum jumps, trajectories and state diffusions which do not exist in usual quantum mechanical formalism but they do exist in the modern experimental quantum physics, can be interpreted in the modern mathematical framework of quantum stochastic processes in terms of the results of the generalized quantum measurements. The problem of quantum measurement which has been always the greatest problem of interpretation of the mathematical formalism of quantum mechanics, is unsolvable in the orthodox formulation of quantum theory. However it has been recently resolved in a more general framework of the algebraic theory of quantum systems which admits the superselection rules for the admissible sets of observables defining the physical systems. The new superselection rule, which we call quantum causality, or nondemolition principle, can be formulated in short as a the following resolution of the corpuscular-wave dualism: the past is classical (encoded into the trajectories of the particles), and the future is quantum (encoded into the propensity waves for these particles). This principle does not apply, it simply does not exist in the usual quantum theory with finite degrees of freedom. And there are no events, jumps and trajectories and other physics in this theory if it is not supplemented with the additional phenomenological interface rules such as projection postulate, permanent reduction or a spontaneous localization theory. This is why it is not applicable for our description of the open quantum world from inside of this world as we were a part of this world, but only for the external description of the whole of a closed physical system as we were outside of this world. However the external description doesn't allow to have a look inside the quantum system as any flow of information from the quantum world which can be obtained only by performing a measurement, will require an external measurement apparatus, and it will inevitable open the system. This is why there is no solution of quantum measurement and all paradoxes of quantum theory in the conventional, external description. 
As we demonstrated in the Section 5 on the simplest quantum measurement model for the Schrödinger's cat, this new superselection rule of quantum causality explains the entanglement and decoherence and derives the projection "postulate" in purely dynamical terms of quantum mechanics of infinitely extended system supplemented with the conventional rules for the statistical inference (statistical prediction by usual conditioning) from the classical information theory and statistics. This provides a solution of the instantaneous quantum measurement problem in its orthodox formulation. The realistic measurements however are not instantaneous but have a duration, and physically are performed even in the continuous time.

Recent dynamical models of the phenomenological theories for quantum jumps and spontaneous localizations, although they all pretend to have a primary value, extend in fact the instantaneous projection postulate to a certain, counting class of the continuous in time measurements. The time which appears in these theories is not the time at which the experimentalist decides to make a measurement on the system, but the time which the system does something for the experimenter to be observed. What it actually does and why, remains unexplained mystery in these theories. As was shown Section 6, there is no need in supplementing the usual quantum mechanics with any of such mysterious quantum spontaneous localization principles even if they are formulated in continuous time. They all have been derived from the time continuous unitary evolution for a generalized Dirac type Schrödinger equation, and 'that something' what the system does to be spontaneously observed, is simply caused by a singular scattering interaction at the boundary of our Hamiltonian model. The quantum causality principle provides a time continuous nondemolition counting measurement in the extended system which enables to obtain 'these stupid quantum jumps' simply by time continuous conditioning called quantum jump filtering.

And even the continuous diffusive trajectories of quantum state diffusion models have been derived from the usual Hilbert space unitary evolution corresponding to the Dirac type boundary value problem for a Schrödinger infinite particles equation with a singular scattering interaction. As it is shown in the Section 7, our causality principle admits to select a continuous diffusive classical process in the quantum extended world which satisfies the nondemolition condition with respect to all future of the measured system. And this allows to obtain the continuous trajectories for quantum state diffusion by simple filtering of quantum noise exactly as it was done in the classical statistical nonlinear filtering and prediction theory. In fact, the quantum state diffusion was first derived over 20 years ago in the result of solving of a similar quantum prediction problem by filtering the quantum white noise in a quantum stochastic Langevin model for the continuous observation and optimal quantum feedback control. Thus the "primary" stochastic nonlinear irreversible quantum state diffusion occurs to be the secondary, as it should be, to the deterministic linear unitary reversible evolution, but in an extended system containing an infinite number of auxiliary particles. And this is quantum causality who defines the arrow of time by selecting what part of the reversible world is related to the classical past and what is related to the quantum future. And this makes the unitary group evolution irreversible in terms of the injective semigroup of the Heisenberg transformations allowing the decoherence and the increase of entropy in a purely dynamical way without any sort of reservoir averaging. 
Our mathematical formulation of the extended quantum mechanics equipped with the quantum causality to allow events and trajectories in the theory, is just as continuous as Schrödinger could have wished. However it doesn't exclude the jumps which only appear in the singular interaction picture, are there as a part of the theory but not only of its interpretation. Although Schrödinger himself didn't believe in quantum jumps, he tried several times, although unsuccessfully, a possibility to obtain the continuous reduction from a generalized, relativistic, "true Schrödinger". He envisaged that "if one introduces two symmetric systems of waves, which are traveling in opposite directions; one of them presumably has something to do with the known (or supposed to be known) state of the system at a later point in time' [102], then it would be possible to derive the 'verdammte Quantenspringerei' for the opposite wave as a solution of the future-past boundary value problem. This desire coincides with the "transactional" attempt of interpretation of quantum mechanics suggested in [103 on the basis that the relativistic wave equation yields in the nonrelativistic limit two Schrödinger type equations, one of which is the time reversed version of the usual equation: "The state vector $\psi$ of the quantum mechanical formalism is a real physical wave with spatial extension and it is identical with the initial "offer wave" of the transaction. The particle (photon, electron, etc.) and the collapsed state vector are identical with the completed transaction.' There was no proof of this conjecture, and now we know that it is not even possible to derive the quantum state diffusions, spontaneous jumps and single reductions from such models involving only a finite particle state vectors $\psi(t)$ satisfying the conventional Schrödinger equation.

Our new approach based on the exactly solvable boundary value problems for infinite particle states described in this paper, resolves this problem formulated by Schrödinger. And thus it resolves the old problem of interpretation of the quantum theory, together with its famous paradoxes in a constructive way by giving exact nontrivial models for allowing the mathematical analysis of quantum observation processes determining the phenomenological coupling constants and the reality underlying these paradoxes. Conceptually it is based upon a new idea of quantum causality called the nondemolition principle [2] which divides the world into the classical past, forming the consistent histories, and the quantum future, the state of which is predictable for each such history. The nondemolition principle defines what is actual in the reality and what is only possible, what are the events and what are just the questions, and selects from the possible observables the actual ones as the candidates for Bell's beables. It was unknown to Bell who wrote that "There is nothing in the mathematics to tell what is 'system' and what is 'apparatus', ...", in [1], p.174). The mathematics of quantum open systems and quantum stochastics defines the extended system by the product of the commutative algebra of the output trajectories, the measured system, and the noncommutative algebra of the input quantum waves. All output processes in the apparatus are the beables which "live" in the center of the algebra, and all other observables which are not in the system algebra, are the input quantum noises of the measurement apparatus whose quantum states are represented by the offer waves. These are the only possible conditions when the posterior states exist as the results of inference (filtering and prediction) of future quantum states upon the measurement results of the classical past as beables. The act of measurement transforms quantum propensities into classical realities. As Lawrence Bragg, another Nobel prize winner, once said, everything in the future is a wave, everything in the past is a particle. 


\section{ApPENDiCES}

5.1. On Bell's "Proof" that von Neumann's Proof was in Error. To "disprove" the von Neumann's theorem on the nonexistence of hidden variables in quantum mechanics Bell [64 argued that the dispersion-free states specified by a hidden parameter $\lambda$ should be additive only for commuting pairs from the space $\mathfrak{L}$ of all Hermitian operators on the system Hilbert space $\mathfrak{h}$. One can assume even less, that the corresponding probability function $E \mapsto\langle E\rangle_{\lambda}$ should be additive with respect to only orthogonal decompositions in the subset $\mathcal{P}(\mathfrak{h})$ of all Hermitian projectors $E$, as only orthogonal events are simultaneously verifiable by measuring an observable $L \in \mathfrak{L}$. In the case of finite-dimensional Hilbert space $\mathfrak{h}$ it is equivalent to the Bell's assumption, but we shall reformulate his only counterexample it terms of the propositions, or events $E \in \mathcal{P}(\mathfrak{h})$ in order to dismiss his argument that this example 'is not dealing with logical propositions, but with measurements involving, for example, differently oriented magnets' (p.6 in 価).

Bell's hidden dispersion-free states were designed to reproduce the regular quantummechanical states of two-dimensional space $\mathfrak{h}=\mathbb{C}^{2}$. The regular pure quantum states are described by one-dimensional projectors

$$
\rho=\frac{1}{2}(\mathrm{I}+\sigma(\mathbf{r})) \equiv P(\mathbf{r}), \quad \sigma(\mathbf{r})=x \sigma_{x}+y \sigma_{y}+z \sigma_{z}
$$

given by the points $\mathbf{r}=x \mathbf{e}_{x}+y \mathbf{e}_{y}+z \mathbf{e}_{z}$ on the unit sphere $\mathbf{S} \subset \mathbb{R}^{3}$ and Pauli matrices $\sigma$. Bell assigned the simultaneously definite values

$$
s_{\lambda}(\mathbf{e})= \pm 1 \equiv\langle\sigma(\mathbf{e})\rangle_{\lambda}, \quad \mathbf{e} \in \mathbf{S}_{\lambda}^{ \pm}(\mathbf{r})
$$

to spin operators $\sigma(\mathbf{e})$ describing the spin projections in the directions $\mathbf{e} \in \mathbf{S}$, which is specified by a split of $\mathbf{S}$ into a positive $\mathbf{S}_{\lambda}^{+}(\mathbf{r})$ and negative $\mathbf{S}_{\lambda}^{-}(\mathbf{r})$ parts depending on the polarization $\mathbf{r}$ and a parameter $\lambda$. Due to

$$
\sigma(-\mathbf{e})=-\sigma(\mathbf{e}), \quad \sigma(\mathbf{e})^{2}=\mathrm{I}
$$

and $\langle\mathrm{I}\rangle_{\lambda}=1$, the values \pm 1 of $s_{\lambda}(\mathbf{e})$ can be taken as dispersion-free expectations $\langle\sigma(\mathbf{e})\rangle_{\lambda}$ of the projections $\sigma(\mathbf{e})$ if $s_{\lambda}(-\mathbf{e})=-s_{\lambda}(\mathbf{e})$. The latter is achieved by a reflection-symmetric partition

$$
\mathbf{S}_{\lambda}^{-}=-\mathbf{S}_{\lambda}^{+}, \quad \mathbf{S}_{\lambda}^{-} \cup \mathbf{S}_{\lambda}^{+}=\mathbf{S}, \quad \mathbf{S}_{\lambda}^{-} \cap \mathbf{S}_{\lambda}^{+}=\emptyset
$$

of the unit sphere $\mathbf{S}$ for each $\mathbf{r}$ and $\lambda$. Obviously there are plenty of such partitions, and any will do, but Bell took a special family

$$
\mathbf{S}_{\lambda}^{ \pm}(\mathbf{r})=\left[\mathbf{S}^{ \pm}(\mathbf{r}) \backslash \mathbf{S}_{\lambda}( \pm \mathbf{r})\right] \cup\left[\mathbf{S}^{\mp}(\mathbf{r}) \backslash \mathbf{S}_{-\lambda}( \pm \mathbf{r})\right],
$$

where $\mathbf{S}^{ \pm}$are south and north hemispheres of the standard reflection-symmetric partition with $\mathbf{r}$ pointing north. Although this particular choice is not better than any other one, he parametrized

$$
\mathbf{S}_{\lambda}(\mathbf{r})=\{\mathbf{e} \in \mathbf{S}: \mathbf{e} \cdot \mathbf{r}<2 \lambda\}
$$

by $\lambda \in\left[-\frac{1}{2}, \frac{1}{2}\right]$ in such a way that

$$
\int_{-1 / 2}^{1 / 2} s_{\lambda}(\mathbf{e}) \mathrm{d} \lambda=\operatorname{Pr}\left\{\lambda: \mathbf{S}_{\lambda}^{+}(\mathbf{r}) \ni \mathbf{e}\right\}-\operatorname{Pr}\left\{\lambda: \mathbf{S}_{\lambda}^{-}(\mathbf{r}) \ni \mathbf{e}\right\}=\mathbf{e} \cdot \mathbf{r} .
$$

Note that in his formula $\mathbf{r}=\mathbf{e}_{z}$, but it can be extended also to the case $|\mathbf{r}| \leq 1$ of not completely polarized quantum states $\rho$ defining the quantum-mechanical 
expectations $\langle\sigma(\mathbf{e})\rangle$ and quantum probabilities $\operatorname{Pr}\{P(\mathbf{e})=1\}$ of the propositions $E=P(\mathbf{e})$ as the linear and affine forms in the unit ball of all such $\mathbf{r}$ :

$$
\operatorname{Tr} \sigma(\mathbf{e}) \rho=\mathbf{e} \cdot \mathbf{r}, \quad \operatorname{Tr} P(\mathbf{e}) \rho=\frac{1}{2}(1+\mathbf{e} \cdot \mathbf{r}) .
$$

Each $\lambda$ assigns the zero-one probabilities $\langle P( \pm \mathbf{e})\rangle_{\lambda}=\chi_{\boldsymbol{\lambda}}^{ \pm}(\mathbf{e})$ given by the characteristic functions $\chi_{\lambda}^{ \pm}$of $\mathbf{S}_{\lambda}^{ \pm}$simultaneously for all quantum events $P( \pm \mathbf{e})$, the eigen-projectors of $\sigma(\mathbf{e})$ corresponding to the eigenvalues \pm 1 :

$$
P( \pm \mathbf{e})=\frac{1}{2}(I \pm \sigma(\mathbf{e})) \mapsto \chi_{\lambda}^{ \pm}(\mathbf{e})=\frac{1}{2}\left(1 \pm s_{\lambda}(\mathbf{e})\right)
$$

The additivity of the probability function $E \mapsto\langle E\rangle_{\lambda}$ in $\mathcal{P}(\mathfrak{h})=\{\mathrm{O}, P(\mathbf{S})$, I $\}$ at each $\lambda$ follows from $\langle\mathrm{O}\rangle_{\lambda}=0$ :

$$
\langle\mathrm{O}\rangle_{\lambda}+\langle\mathrm{I}\rangle_{\lambda}=1=\langle\mathrm{O}+\mathrm{I}\rangle_{\lambda}
$$

as $\mathrm{O}+\mathrm{I}=\mathrm{I}$, and from $\chi_{\lambda}^{+}(-\mathbf{e})=\chi_{\lambda}^{-}(\mathbf{e})$ :

$$
\langle P(\mathbf{e})\rangle_{\lambda}+\langle P(-\mathbf{e})\rangle_{\lambda}=1=\langle P(\mathbf{e})+P(-\mathbf{e})\rangle_{\lambda},
$$

as $P(\mathbf{e})+P(-\mathbf{e})=\mathrm{I}$.

Thus a classical hidden variable theory reproducing the affine quantum probabilities $\mathrm{P}(\mathbf{e})=\langle P(\mathbf{e})\rangle$ as the uniform mean value

$$
\mathrm{M}\langle P(\mathbf{e})\rangle .=\int_{-1 / 2}^{1 / 2} \frac{1}{2}\left(1+s_{\lambda}(\mathbf{e})\right) \mathrm{d} \lambda=\frac{1}{2}(1+\mathbf{e} \cdot \mathbf{r})=\operatorname{Tr} P(\mathbf{e}) \rho
$$

of the classical yes-no observables $\chi^{+}(\mathbf{e})=\langle P(\mathbf{e})\rangle$. was constructed by Bell.

First let us note that it does not contradict to the von Neumann theorem even if the latter is strengthened by the restriction of the additivity only to the orthogonal projectors $E \in \mathcal{P}(\mathfrak{h})$. The constructed dispersion-free expectation function $\mathrm{L} \mapsto$ $\langle\mathrm{L}\rangle_{\lambda}$ is not physically continuous on $\mathfrak{L}$ because the value $\langle\mathrm{L}\rangle_{\lambda}=s_{\lambda}(\mathbf{l})$ is one of the eigenvalues \pm 1 for each $\lambda$, and it covers both values when the directional vector $\mathbf{l}$ rotates continuously over the three-dimensional sphere. A function $\mathbf{l} \mapsto\langle\sigma(\mathbf{l})\rangle_{\lambda}$ on the continuous manifold (sphere) with discontinuous values can be continuous only if it is constant, but this is ruled out by the impossibility to reproduce the expectations $\langle\sigma(\mathbf{l})\rangle=\mathbf{l} \cdot \mathbf{r}$, which are linear in $\mathbf{l}$, by averaging the function $\lambda \mapsto$ $\langle\sigma(\mathbf{l})\rangle_{\lambda}$, constant in $\mathbf{l}$, over the $\lambda$. Measurements of the projections of spin on the physically close directions should be described by close expected values in any physical state specified by $\lambda$, otherwise it cannot have physical meaning!

Indeed, apart from partial additivity (the sums are defined in $\mathcal{P}(\mathfrak{h})$ only for the orthogonal pairs from $\mathcal{P}(\mathfrak{h}))$, the von Neumann theorem restricted to $\mathcal{P}(\mathfrak{h}) \subset \mathfrak{L}$ should also inherit the physical continuity, induced by ultra-strong topology in $\mathfrak{L}$. In the finite dimensional case it is just ordinary continuity in the projective topology $\mathfrak{h}$, and in the case $\operatorname{dim} \mathfrak{h}=2$ it is the continuity on the projective space $\mathbf{S}$ of all one-dimensional projectors $P(\mathbf{e}), \mathbf{e} \in \mathbf{S}$. It is obvious that the zero-one probability function $E \mapsto\langle E\rangle_{\lambda}$ constructed by Bell is not physically continuous on the restricted set: the characteristic function $\chi_{\lambda}^{+}(\mathbf{e})=\langle P(\mathbf{e})\rangle_{\lambda}$ of the half-sphere $\mathbf{S}_{\lambda}^{+}(\mathbf{r})$ is discontinuous in $\mathbf{e}$ on the whole sphere $\mathbf{S}$ for any $\lambda$ and $\mathbf{r}$. Measurements of the spin projections in the physically close directions $\mathbf{e}_{n} \rightarrow \mathbf{e}$ should be described by close probabilities $\left\langle P\left(\mathbf{e}_{n}\right)\right\rangle_{\lambda} \rightarrow\langle P(\mathbf{e})\rangle_{\lambda}$ in any physical state specified by $\lambda$, otherwise the state cannot have physical meaning! 
The continuity argument might be considered to be as purely mathematical, but in fact it is not: even in classical probability theory with a discrete phase space the pure states defined by Dirac $\delta$-measure, are uniformly continuous, as any positive probability measure is on the space of classical observables defined by bounded measurable functions on any continuous phase space. In quantum theory an expectation defined as a linear positive functional on $\mathfrak{L}$ is also uniformly continuous, hence the von Neumann assumption of physical (ultra-weak) continuity is only a restriction in the infinite-dimensional case. Even if the state is defined only on $\mathcal{P}(\mathfrak{h}) \subset \mathfrak{L}$ as a probability function which is additive only on the orthogonal projectors, the uniform continuity follows from its positivity in the case of $\operatorname{dim} \mathfrak{h} \geq 3$. This follows from the Gleason's theorem [65] with implication that Bell's dispersionfree states could exist only if the Hilberet space of our whole universe had the dimensionality not more than two.

In fact, Gleason obtained more than this: He proved that the case $\operatorname{dim} \mathfrak{h}=2$ is the only exceptional one when a probability function on $\mathcal{P}(\mathfrak{h})$ (which should be countably additive in the case $\operatorname{dim} \mathfrak{h}=\infty$ ) may not be induced by a density operator $\rho$, and thus cannot be extended to a linear expectation on the operator space $\mathfrak{L}$. The irregular states cannot be extended by linearity on the algebra of all (not just Hermitian) operators in $\mathfrak{h}=\mathbb{C}^{2}$ even if they are continuous.

To rule out even this exceptional case form the Gleason's theorem we note that an irregular state $E \mapsto\langle E\rangle$ on $\mathcal{P}\left(\mathbb{C}^{2}\right)$ cannot be composed with any state of an additional quantum system even if the latter is given by a regular probability function $\langle F\rangle=\operatorname{Tr} F \sigma$ on a set $\mathcal{P}(\mathfrak{f})$ of ortho-projectors of another Hilbert space. There is no additive probability function on the set $\mathcal{P}\left(\mathbb{C}^{2} \otimes \mathfrak{f}\right)$ of all verifiable events for the compound quantum system described by a nontrivial Hilbert space $\mathfrak{f}$ such that

$$
\langle E\rangle=\langle E \otimes \mathrm{I}\rangle, \quad\langle\mathrm{I} \otimes F\rangle=\operatorname{Tr} F \sigma,
$$

where $\sigma=P_{\varphi}$ is the density operator of wave function $\varphi \in \mathfrak{f}$. Indeed, if it could be possible for some $\mathfrak{f}$ with $\operatorname{dim} \mathfrak{f}>1$, it would be possible for $\mathfrak{f}=\mathbb{C}^{2}$. By virtue of Gleason's theorem any probability function which is additive for orthogonal projectors on $\mathbb{C}^{2} \otimes \mathbb{C}^{2}=\mathbb{C}^{4}$ is regular on $\mathcal{E}\left(\mathbb{C}^{4}\right)$, given by a density operator $\hat{\varrho}$. Hence

$$
\langle E\rangle=\operatorname{Tr}(\mathrm{I} \otimes E) \hat{\varrho}=\operatorname{Tr} E \rho
$$

i.e. the state on $\mathcal{P}\left(\mathbb{C}^{2}\right)$ is also regular, with the density operator in $\mathfrak{h}=\mathbb{C}^{2}$ given by the partial trace

$$
\rho=\operatorname{Tr}[\hat{\varrho} \mid \mathfrak{h}]=\operatorname{Tr}_{\mathfrak{f}} \hat{\varrho} .
$$

In order to obtain an additive product-state on $\mathcal{P}\left(\mathbb{C}^{2} \otimes \mathfrak{f}\right)$ satisfying

$$
\langle E \otimes F\rangle=\langle E\rangle \operatorname{Tr} F P_{\varphi}, \quad E \in \mathcal{P}\left(\mathbb{C}^{2}\right), F \in \mathcal{P}(\mathfrak{f})
$$

for a finite-dimensional $\mathfrak{f}=\mathbb{C}^{n}$ with $n>1$ it is necessary to define the state as an expectation on the whole unit ball $\mathcal{B}\left(\mathbb{C}^{2}\right)$ of the algebra $\mathfrak{B}=\mathcal{B}\left(\mathbb{C}^{2}\right)$ of all (not just Hermitian) operators in $\mathbb{C}^{2}$. Indeed, any one-dimensional Hermitian projector in $\mathbb{C}^{2} \otimes \mathbb{C}^{n}=\mathbb{C}^{2 n}$ can be described as an $n \times n$-matrix $\mathbf{E}=\left[\mathrm{A}_{j} \mathrm{~A}_{i}^{\dagger}\right]$ with $2 \times 2$-entries 
$\mathrm{A}_{j} \in \mathcal{B}\left(\mathbb{C}^{2}\right), j=1, \ldots, n$ satisfying the normalization condition

$$
\sum_{j=1}^{n} \mathrm{~A}_{j}^{\dagger} \mathrm{A}_{j}=P(\mathbf{e})=\frac{1}{2}(I+\sigma(\mathbf{e}))
$$

for some $\mathbf{e} \in \mathbf{S}$. These entries have the form

$$
\mathrm{A}=\alpha P(\mathbf{e})+a Q\left(\mathbf{e}_{\perp}\right), \quad Q\left(\mathbf{e}_{\perp}\right)=\frac{1}{2} \sigma\left(\mathbf{e}_{\perp}\right),
$$

where $\mathbf{e}_{\perp}$ is an orthogonal complex vector such that

$$
i \mathbf{e}_{\perp} \times \mathbf{e}=\mathbf{e}_{\perp} \quad \overline{\mathbf{e}}_{\perp} \cdot \mathbf{e}_{\perp}=2, \quad i \overline{\mathbf{e}}_{\perp} \times \mathbf{e}_{\perp}=2 \mathbf{e},
$$

and $\sum\left(\left|\alpha_{j}^{2}\right|+\left|a_{j}^{2}\right|\right)=1$ corresponding to $\operatorname{Tr} \mathbf{E}=1$. The matrix elements

$$
\mathrm{A}_{j} \mathrm{~A}_{i}^{\dagger}=\alpha_{j} \bar{\alpha}_{i} P(\mathbf{e})+a_{j} \bar{a}_{i} P(-\mathbf{e})+\alpha_{j} \bar{a}_{i} Q\left(\overline{\mathbf{e}}_{\perp}\right)+a_{j} \bar{\alpha}_{i} Q\left(\mathbf{e}_{\perp}\right)
$$

for these orthoprojectors in $\mathbb{C}^{2 n}$ are any matrices from the unit ball $\mathcal{B}\left(\mathbb{C}^{2}\right)$, not just Hermitian orthoprojectors. By virtue of Gleason's theorem the product-state of such events $\mathbf{E}$ must be defined by the additive probability

$$
\langle\mathbf{E}\rangle=\sum_{i, j=1}^{n} \varphi^{j} \varrho\left(\mathrm{A}_{j} \mathrm{~A}_{\iota}^{\dagger}\right) \varphi^{i}=\varrho(\mathrm{B}),
$$

where $\mathrm{B}=\mathrm{A}(\varphi) \mathrm{A}(\varphi)^{\dagger}=\beta I+\sigma(\mathbf{b})$ is given by $\alpha(\varphi)=\varphi^{j} \alpha_{j}, a(\varphi)=\varphi^{j} a_{j}$ for $\varphi \in \mathbb{C}^{n}$ with the components $\varphi^{j}=\bar{\varphi}_{j}$, and $\varrho(\mathrm{B})=\operatorname{Tr} \mathrm{B} \rho$ is the linear expectation

$$
\varrho(\mathrm{B})=\frac{1}{2}\left(\beta_{+}\left(1+r_{1}\right)+\beta_{-}\left(1-r_{1}\right)+b_{\perp} \bar{r}_{\perp}+\bar{b}_{\perp} r_{\perp}\right)=\beta+\mathbf{b} \cdot \mathbf{r}
$$

with $r_{1}=\mathbf{e} \cdot \mathbf{r}, \mathbf{r}_{\perp}=\mathbf{e}_{\perp} \cdot \mathbf{r}, \beta_{+}=|\alpha(\varphi)|^{2}, \beta_{-}=|a(\varphi)|^{2}, b_{\perp}=\alpha(\varphi) \overline{a(\varphi)}$. It these terms we can formulate the definition of a regular state without assuming a priori the linearity and even continuity conditions also for the case $\mathfrak{h}=\mathbb{C}^{2}$.

Thus we proved that in order to formulate the quantum composition principle for a physical system described by a Hilbert space $\mathfrak{h}$ we need the quantum state to be defined on the unite ball $\mathcal{B}(\mathfrak{h})$ rather than just on the set $\mathcal{P}(\mathfrak{h})$ of the orthoprojectors in $\mathfrak{h}$. The following definition obviously rules out the Bell's hidden variable states even in the case $\mathfrak{h}=\mathbb{C}^{2}$ as unphysical.

A complex-valued map $\mathrm{B} \mapsto \varrho(\mathrm{B})$ on the unit ball $\mathcal{B}(\mathfrak{h})$ normalized as $\varrho(\mathrm{I})=1$ is called state for a quantum system described by the Hilbert space $\mathfrak{h}$ (including the case $\operatorname{dim} \mathfrak{h}=2)$ if it is positive on all Hermitian projective matrices $\mathbf{E}=\left[\mathrm{A}_{j} \mathrm{~A}_{k}^{\dagger}\right]$ with entries $\mathrm{A}_{j} \in \mathcal{B}(\mathfrak{h})$ in the sense

$$
\sum_{j} \mathrm{~A}_{j}^{\dagger} \mathrm{A}_{j}=P \in \mathcal{P}(\mathfrak{h}) \Rightarrow \varrho(\mathbf{E})=\left[\varrho\left(\mathrm{A}_{j} \mathrm{~A}_{k}^{\dagger}\right)\right] \geq 0
$$

of positive-definiteness of the matrices $\varrho(\mathbf{E})$ with the complex entries $\left[\varrho\left(\mathrm{A}_{j} \mathrm{~A}_{k}^{\dagger}\right)\right]$. It is called a regular state if

$$
\varrho\left(E \otimes P_{\varphi}\right)=\varrho(E) P_{\varphi}
$$


for any one-dimensional projector $P_{\varphi}=\left[\varphi_{j} \varphi_{i}^{\dagger}\right]$, and if it is countably-additive with respect to the orthogonal decompositions $\mathbf{E}=\sum \mathbf{E}(k)$ :

$$
\sum_{j} \mathrm{~A}_{j}(i)^{\dagger} \mathrm{A}_{j}(k)=0, \forall i \neq k \Rightarrow \varrho\left(\sum_{k} \mathrm{~A}_{j}(k) \mathrm{A}_{j}(k)^{\dagger}\right)=\sum_{k} \varrho\left(\mathrm{A}_{j}(k) \mathrm{A}_{i}(k)^{\dagger}\right) .
$$

It obvious that the state thus defined can be uniquely extended to a regular product-state on $\mathcal{P}\left(\mathfrak{h} \otimes \mathbb{C}^{n}\right)$ by

$$
\sum_{j, k} \bar{\varphi}_{j} \varrho\left(\mathrm{A}_{j} \mathrm{~A}_{i}^{\dagger}\right) \varphi_{i} \geq 0, \quad \forall \varphi_{j} \in \mathbb{C}, \quad \sum\left|\varphi_{j}\right|=1,
$$

which proves that it is continuous and is given by a density operator: $\varrho(\mathrm{B})=$ $\operatorname{Tr} \mathrm{B} \rho$. Thus the composition principle rules out the existence of the hidden variable representation for the quantum systems.

In conclusion we note that the Bells example in fact doesn't prove the existence of hidden variables from any reasonable probabilistic point of view even in this exceptional case $\mathfrak{h}=\mathbb{C}^{2}$. Indeed, his mean $\mathrm{M}$ over $\lambda$ cannot be considered as the conditional averaging of a classical partially hidden world with respect to the quantum observable part. If this were so, not only the mean values of the classical hidden variables but also their momenta would reproduce the regular quantum expectations as linear functionals of $\rho$. Bell's model however gives nonlinear expectations with respect to the states $\rho$ even if it is restricted to the smallest commutative algebra generated by the characteristic functions $\left\{\chi_{.^{+}}(\mathbf{e}): \mathbf{e} \in \mathbf{S}\right\}$ of the subsets $\left\{\lambda: \mathbf{S}_{\lambda}^{+}(\mathbf{r}) \ni \mathbf{e}\right\}$. One can see this by the uniform averaging of the commutative products $\chi_{\lambda}^{+}(\mathbf{e}) \chi_{\lambda}^{+}(\mathbf{f})$ : such mean values (i.e. the second order moments) are affine with respect to $\mathbf{r}$ only for colinear $\mathbf{e}$ and $\mathbf{f} \in \mathbf{S}$.

5.2. Quantum Markov Chains and Stochastic Recurrences. Let $\mathfrak{h}, \mathfrak{g}_{o}$ be Hilbert spaces, $\mathrm{V}$ be an isometry $\mathfrak{h} \mapsto \mathfrak{g}_{o} \otimes \mathfrak{h}, \mathrm{V}^{\dagger} \mathrm{V}=\mathrm{I}$, and $\mathcal{A}$ and $\mathcal{B}$ be von Neumann algebras respectively on $\mathfrak{g}$ and $\mathfrak{h}$ and there exists a normal representation $\iota$ of the commutant $\mathcal{B}^{\prime}=\left\{\mathrm{B}^{\prime}:\left[\mathrm{B}^{\prime}, \mathrm{B}\right]=0, \forall \mathrm{B} \in \mathcal{B}\right\}$ into the commutant of $\mathcal{A} \otimes \mathcal{B}$ which is intertwined by this isometry:

$$
\iota\left(\mathrm{B}^{\prime}\right) \mathrm{V}=\mathrm{VB}^{\prime} \quad \forall \mathrm{B}^{\prime} \in \mathcal{B}^{\prime} .
$$

The pair $(\mathrm{V}, \iota)$ defines the standard representation of a $\mathcal{B}$-transitional probability map

$$
\pi(\mathrm{A}, \mathrm{B})=\mathrm{V}^{\dagger}(\mathrm{A} \otimes \mathrm{B}) \mathrm{V} \in \mathcal{B} \quad \forall \mathrm{A} \in \mathcal{A}_{o}, \mathrm{~B} \in \mathcal{B}
$$

with an output algebra $\mathcal{A}$. The transitional maps with the output $\mathcal{A}=\mathcal{B}$ on the Hilbert space copy $\mathfrak{g}=\mathfrak{h}$ were introduced by Accardi 106 for the case $\mathcal{B}=\mathcal{B}(\mathfrak{h})$ (in this simple case one can take $\mathcal{A}=\mathcal{B}(\mathfrak{g} \otimes \mathfrak{h})$ and $\iota(\alpha \mathrm{I})=\alpha \mathrm{I} \otimes \mathrm{I}$ on the onedimensional algebra $\mathcal{B}^{\prime}=\mathbb{C I}$ ). Note that the intertwining commutant condition (5.1) which was established for any conditional state in [105] is nontrivial if only $\mathcal{B}$ is smaller than the whole operator algebra $\mathcal{B}(\mathfrak{h})$. Every normal completely positive unital map $\kappa: \mathcal{A} \rightarrow \mathcal{B}$ can be extended to a $\mathcal{B}$-transitional probability map in the standard representation of $\mathcal{A}$ such that $\kappa(\mathrm{A})=\mathrm{V}^{\dagger}(\mathrm{I} \otimes \mathrm{A}) \mathrm{V}$ 105. 

if

We shall say that the $\mathcal{B}$-transitional map $\pi$ is given in a stochastic representation

$$
\pi(\mathrm{A}, \mathrm{B})=\mathrm{F}^{\dagger}(\tau(\mathrm{A} \otimes \mathrm{B})) \mathrm{F} \in \mathcal{B} \quad \forall \mathrm{A} \in \mathcal{A}, \mathrm{B} \in \mathcal{B},
$$

where $\mathrm{F}$ is an isometry $\mathfrak{h} \rightarrow \mathfrak{f} \otimes \mathfrak{h}$ from $\mathfrak{f} \otimes \mathcal{B}$ in the sense $\mathrm{FB}^{\prime}=\left(\mathrm{I} \otimes \mathrm{B}^{\prime}\right) \mathrm{F}$ for all $\mathrm{B}^{\prime} \in \mathcal{B}^{\prime}$, and $\tau$ is a normal, not necessarily unital representation of $\mathcal{A}$ in the algebra $\mathcal{B}(\mathfrak{f}) \otimes \mathcal{B}$. Every normal completely positive unital map $\kappa: \mathcal{A} \rightarrow \mathcal{B}$ has a stochastic representation ( 104, p. 61) with $\mathrm{F}=\mathrm{T}^{\dagger} \mathrm{V}$ and $\tau(\mathrm{A})=\mathrm{T}^{\dagger} \mathrm{AT}$ given by a partial isometry $T: \mathfrak{f} \otimes \mathfrak{h} \rightarrow \mathfrak{g} \otimes \mathfrak{h}$. such that

$$
\mathrm{T}\left(\mathrm{I} \otimes \mathrm{B}^{\prime}\right) \mathrm{T}^{\dagger}=\iota\left(\mathrm{B}^{\prime}\right), \quad \forall \mathrm{B}^{\prime} \in \mathcal{B}^{\prime}
$$

The representations (5.2) and (5.3) are called respectively stochastic and standard if $\mathfrak{g}=\mathfrak{h} \otimes \mathfrak{k}=\mathfrak{f}$ and

$$
\left(\mathrm{B}^{\prime} \otimes \mathrm{A}_{o} \otimes \mathrm{B}\right) \mathrm{T}=\mathrm{T}\left(\mathrm{B} \otimes \mathrm{A}_{o} \otimes \mathrm{B}^{\prime}\right), \quad \forall \mathrm{A}_{o} \in \mathcal{A}_{o}, \mathrm{~B}^{\prime} \in \mathcal{B}^{\prime} .
$$

such that $\mathcal{A}=\mathrm{I} \otimes \mathcal{A}_{o}$ and $\iota\left(\mathrm{B}^{\prime}\right)=\mathrm{B}^{\prime} \otimes \mathrm{I}$ (e.g. $\mathrm{T}$ is tensor transposition $\mathrm{T}(\varphi \otimes \chi \otimes \psi)=$ $\psi \otimes \chi \otimes \varphi)$. Every normal completely positive unital map $\pi$ has a standard stochastic representation if it is dominated by a normal faithful state (or weight) on $\mathcal{A}_{o} \otimes \mathcal{B}$ in the sense of 109 . If $\mathfrak{g}=L_{\mu}^{2}$ and $\mathcal{B}$ is commutative, the diagonal algebra $\mathcal{B}=\mathcal{D}(\mathfrak{h}) \simeq L_{\mu}^{\infty}$ on the space $\mathfrak{h}=L_{\mu}^{2}$ of square-integrable function $\psi(y)$ say, the conditional probability in the standard stochastic representation is described by the transposition $\mathrm{V}=\mathrm{T}_{o} \hat{v}$ of the operator

$$
(\hat{v} \psi)(y, x)=v(y, x) \psi(y), \quad \int|\nu(y, x)|^{2} \mathrm{~d} \mu_{x}=1
$$

from $L_{\mu}^{2}$ into $L_{\mu}^{2} \otimes \mathfrak{g}$, the multiplication by a measurable function $v$ with normalized vector values $v(y) \in \mathfrak{g},\|v(y)\|=1$ for almost all $y$ :

$$
(\mathrm{V} \psi)(x, y)=\left(\mathrm{T}_{o} \hat{v} \psi\right)(x, y)=(\hat{v} \psi)(x, y)=v(x, y) \psi(x), \quad \forall \psi \in L_{\mu}^{2} .
$$

The conditional probability amplitude $v$ defines the usual conditional probability with the density $|v(x, y)|^{2}$ for the transitions $y_{0}=x \mapsto y$ with respect to a given measure $\mu$, and only such conditional probabilities which are absolutely continuous have standard stochastic representation.

Every conditional probability map defines a quantum $\mathcal{B}$-Markov chain

$$
\pi\left(t, \mathrm{~A}^{1}, \ldots, \mathrm{A}^{t}, \mathrm{~B}\right)=\mathrm{V}^{\dagger}\left(\mathrm{A}^{1} \otimes \cdots\left(\mathrm{V}^{\dagger}\left(\mathrm{A}^{t} \otimes \mathrm{B}\right) \mathrm{V}\right) \ldots\right) \mathrm{V}, \quad t \in \mathbb{N}
$$

with an output algebra $\mathcal{A}_{o} \subseteq \mathcal{B}(\mathfrak{g})$ over an operator algebra $\mathcal{B} \subseteq \mathcal{B}(\mathfrak{h})$. Quantum Markov chains with an output were introduced in [3] as effectively described by the quantum recurrency equation

$$
\pi\left(t, \mathrm{~A}_{0}^{t]}, \mathrm{B}\right)=\mathrm{V}(t)^{\dagger}\left(\mathrm{A}^{1} \otimes \cdots \otimes \mathrm{A}^{t} \otimes \mathrm{B}\right) \mathrm{V}(t)=\pi\left(t-1, \mathrm{~A}_{0}^{t-1]}, \mathrm{V}^{\dagger}\left(\mathrm{A}^{t} \otimes \mathrm{B}\right) \mathrm{V}\right)
$$

(see also an earlier definition in [106 for the simple standard case $\mathcal{B}=\mathcal{B}(\mathfrak{h})$ ). Here $\mathrm{A}_{0}^{t]}=\left(\mathrm{A}^{1}, \ldots, \mathrm{A}^{t}\right)$ is a sequence of indexed elements $\mathrm{A}^{t} \in \mathcal{A}_{o}$ and $\mathrm{V}(t)=$ $\mathrm{V}_{t} \mathrm{~V}(t-1)$ with $\mathrm{V}(0)=\mathrm{I}$ is the isometry $\mathfrak{h} \rightarrow \mathfrak{g}_{0}^{t]} \otimes \mathfrak{h}$, where $\mathfrak{g}_{0}^{t]}=\otimes_{0<r \leq t} \mathfrak{g}_{s}$ is the tensor product of the copies of $\mathfrak{g}_{o}$.. This isometry is given by the recurrency equation

$$
\mathrm{V}(t)=\mathrm{V}_{t} \mathrm{~V}(t-1), \quad \mathrm{V}(0)=\mathrm{I}
$$


defined by $\mathrm{V}_{t}=\mathrm{I}_{0}^{t} \otimes \mathrm{V}$, where $\mathrm{I}_{0}^{t}=\otimes_{0<r<t} \mathrm{I}_{r}$ with all $\mathrm{I}_{s}=\mathrm{I}_{o}$ is the identity in $\mathfrak{g}_{0}^{t}=\otimes_{0<r<t} \mathfrak{g}_{r}$. In particular, if there is no output, $\mathcal{A}_{o}=\mathbb{C}$ corresponding to $\mathfrak{g}=\mathbb{C}$, the operator $\mathrm{V}$ is an isometry $\mathfrak{h} \rightarrow \mathfrak{h}$ which is often unitary, $\mathrm{V}^{\dagger}=\mathrm{V}^{-1}$, as it is always in the case of finite dimensional $\mathfrak{h}$. The usual, Heisenberg dynamics $\pi(t, \mathrm{~B})=\mathrm{V}^{-t} \mathrm{BV}^{t}$ induced by the unitary $\mathrm{V}$ in $\mathfrak{h}$ over the algebra $\mathcal{B}=\mathcal{B}(\mathfrak{h})$ doesn't admit a nontrivial output $\mathcal{A}_{o} \neq \mathbb{C}$. Note that Markov chains over the maximal commutative algebras $\mathcal{A}=\mathcal{C}=\mathcal{B}$ on $\mathfrak{g}=L_{\mu}^{2}=\mathfrak{h}$, given as the standard stochastic representation by $\mathrm{V}=\hat{v}$, are equivalent to the classical Markov chains described by the conditional probability densities $p(y \mid x)=|v(y \mid x)|^{2}$ with the standard classical output $\mathcal{A}_{o}=\mathcal{C} \simeq L_{\mu}^{\infty}$.

Now we shall prove that every Markov chain with an output $\mathcal{A}$ and arbitrary $\mathcal{B}$ can be induced by a Heisenberg dynamics on the infinite tensor product algebra $\mathfrak{B}=\mathfrak{A}^{0]} \otimes \mathcal{B} \otimes \mathfrak{A}_{0}$ embedding all tensor powers $\mathcal{A}_{o}^{\otimes t}$ into the output algebra $\mathfrak{A}^{0]}=$ $\otimes_{s \leq 0} \mathcal{A}_{s}$ as $I^{s} \otimes \mathcal{A}_{0}^{\otimes t}$ by $\mathcal{A}_{s}=\mathbb{C} \oplus \mathcal{A}_{o}, s \leq 0$. This induction can be achieved by a unitary semigroup $U^{t}$ for all $t \in \mathbb{N}$ as

$\pi\left(t, \mathrm{~A}_{0}^{t]}, \mathrm{B}\right)=\left(\Phi^{0]} \otimes \mathrm{I} \otimes \Phi_{0}^{\circ}\right)^{\dagger} U^{-t}\left(I^{t} \otimes \mathrm{A}^{1} \otimes \ldots \otimes \mathrm{A}^{t} \otimes \mathrm{B} \otimes I_{0}\right) U^{t}\left(\Phi^{0]} \otimes \mathrm{I} \otimes \Phi_{0}^{\circ}\right)$,

where $\Phi_{0}^{\circ}=\otimes_{s>0} \chi_{s}^{\circ}$ is the input vacuum-vector given by the infinite tensor product of the copies of a unit vector $\chi^{\circ} \in \mathfrak{f}$, and $\Phi^{0]}$ is arbitrary (normalized) in the output Hilbert space $\mathcal{G}^{0]}=\otimes_{s \leq 0} \mathfrak{g}_{s}$ with all $\mathfrak{g}_{s}=\mathfrak{g}$. In the case of commutative $\mathcal{A}=\mathcal{D}(\mathfrak{g})$ this construction will provide the dynamical solution to the quantum measurement problem as stated in the Section 5. We shall give an explicit, canonical construction of this dilation. Other constructions are also possible and known in the simple case $\mathcal{B}=\mathcal{B}(\mathfrak{h})$ at least for finite-dimensional $\mathfrak{h} 107$, 108. In general we need an extension of the spaces $\mathfrak{g}$ and $\mathfrak{f}$, and this is why we denote them here as $\mathfrak{g}_{o}$ and $\mathfrak{f}_{o}$. For simplicity we shall also assume that the stochastic and standard representations are intertwined by a unitary operator $\mathrm{T}$ called transposition and denoted also as $\mathrm{T}_{o}$.

First, let us consider the canonical unitary dilation of the stochastic isometry $\mathrm{V}$ intertwining the algebras $\iota\left(\mathcal{B}^{\prime}\right)$ and $\mathcal{B}^{\prime}$. It is uniquely defined on $\mathfrak{h} \oplus\left(\mathfrak{h} \otimes \mathfrak{f}_{o}\right)$ up to a unitary transformation $\mathrm{e}^{-i \mathrm{E} / \hbar} \in \mathcal{B}$ by

$$
\mathrm{U}=\left[\begin{array}{cc}
\mathrm{O} & \mathrm{V}^{\ddagger} \\
\mathrm{V} & \mathrm{G}
\end{array}\right], \quad \mathrm{G}=\left(\mathrm{I}_{o} \otimes \mathrm{e}^{-i \mathrm{E} / \hbar}\right) \mathrm{T}_{o}-\mathrm{Ve}^{i \mathrm{E} / \hbar} \mathrm{V}^{\ddagger},
$$

where $\mathrm{V}^{\ddagger}=\mathrm{V}^{* \dagger}$ and $\mathrm{V}^{*}=\mathrm{T}_{o}^{\dagger}\left(\mathrm{I} \otimes \mathrm{e}^{i \mathrm{E} / \hbar}\right) \mathrm{Ve}^{i \mathrm{E} / \hbar}$. Here for the notational convenience $U$ is represented as an operator on $\mathfrak{h} \otimes \mathfrak{f}$ into $\mathfrak{g} \otimes \mathfrak{h}$ with $\mathfrak{f}=\mathbb{C} \oplus \mathfrak{f}_{o}$ and $\mathfrak{g}=\mathbb{C} \oplus \mathfrak{g}_{o}$ which is done by the "transposition" $\mathrm{T}_{o}: \mathfrak{h} \otimes \mathfrak{f}_{o} \rightarrow \mathfrak{g}_{o} \otimes \mathfrak{h}$ intertwining the algebra and $\mathcal{B} \otimes \mathrm{I}_{o}$ and $\mathrm{I}_{o} \otimes \mathcal{B}$ as in the standard stochastic case, where $\mathfrak{f}_{o}=\mathfrak{k} \otimes \mathfrak{h}$ and $\mathfrak{g}_{o}=\mathfrak{h} \otimes \mathfrak{k}$.. The unitarity $\mathrm{U}^{-1}=\mathrm{U}^{\dagger}$ of the operator $\mathrm{U}$ follows from $\mathrm{T}^{\dagger} \mathrm{T}=\mathrm{I} \oplus\left(\mathrm{I} \otimes \mathrm{I}_{o}\right)=\mathrm{I} \otimes \mathrm{I}$,

$$
\mathrm{U}^{\dagger} \mathrm{U}=\left[\begin{array}{ll}
\mathrm{O} & \mathrm{V}^{\dagger} \\
\mathrm{V}^{*} & \mathrm{G}^{\dagger}
\end{array}\right]\left[\begin{array}{ll}
\mathrm{O} & \mathrm{V}^{\ddagger} \\
\mathrm{V} & \mathrm{G}
\end{array}\right]=\left[\begin{array}{ll}
\mathrm{V}^{\dagger} \mathrm{V} & \mathrm{V}^{\dagger} \mathrm{G} \\
\mathrm{G}^{\dagger} \mathrm{V} & \mathrm{V}^{*} \mathrm{~V}^{\ddagger}+\mathrm{G}^{\dagger} \mathrm{G}
\end{array}\right]=\left[\begin{array}{ll}
\mathrm{I} & \mathrm{O} \\
\mathrm{O} & \mathrm{I} \otimes \mathrm{I}_{o}
\end{array}\right]
$$

and the selfadjointness of $\mathrm{S}=\mathrm{e}^{i \mathrm{E} / \hbar} \mathrm{T}^{\dagger} \mathrm{U}$. Moreover, as it follows straightforward from the construction of $\mathrm{U}$,

$$
\left(\mathrm{B}^{\prime} \oplus \iota^{-1}\left(\mathrm{~B}^{\prime}\right)\right) \mathrm{U}=\mathrm{U}\left(\mathrm{B}^{\prime} \oplus\left(\mathrm{B}^{\prime} \otimes \mathrm{I}_{o}\right)\right)=\mathrm{U}\left(\mathrm{B}^{\prime} \otimes \mathrm{I}\right) \quad \forall \mathrm{B}^{\prime} \in \mathcal{B}^{\prime}
$$


on $\mathfrak{h} \otimes \mathfrak{f}$, where $\mathrm{I}=1 \oplus \mathrm{I}_{o}$ is the identity on $\mathfrak{f}$. This implies $\mathrm{U}^{\dagger}(\mathrm{A} \otimes \mathrm{B}) \mathrm{U} \in\left(\mathcal{B}^{\prime} \otimes \mathrm{I}\right)^{\prime}$ i.e. $\mathrm{U}^{\dagger}\left(\mathcal{A}_{0} \otimes \mathcal{B}\right) \mathrm{U} \subseteq \mathcal{B} \otimes \mathcal{A}_{1}$ in the sense

$$
\mathrm{U}^{\dagger}(\mathrm{A} \otimes \mathrm{B}) \mathrm{U} \in \mathcal{B} \otimes \mathcal{A}_{1}, \quad \forall \mathrm{A} \in \mathcal{A}_{0}, \mathrm{~B} \in \mathcal{B},
$$

where $\mathcal{A}_{0}=\mathbb{C} \oplus \mathcal{A}_{o}$ and $\mathcal{A}_{1}=\mathcal{B}(\mathfrak{f})$. One can always obtain such $\mathrm{U}$ as $\mathrm{U}=\mathrm{TW}$ from its stochastic representation $\mathrm{W} \in \mathcal{B} \otimes \mathcal{B}(\mathfrak{f})$ as commuting with all $\mathrm{B}^{\prime} \otimes \mathrm{I}$ by the "transposition" $\mathrm{T}=\mathrm{I} \oplus \mathrm{T}_{o}$ of $\mathfrak{h} \otimes \mathfrak{f}$ onto $\mathfrak{g} \otimes \mathfrak{h}$.

Second, we construct the local auxiliary spaces $\mathfrak{g}_{r}^{t]}$ as the Hilbert tensor products $\otimes_{r<z \leq t} \mathfrak{g}_{z}$ of the copies $\mathfrak{g}_{z}=\mathfrak{f}$ for $z>0$ and $\mathfrak{g}_{z}=\mathfrak{g}$ for $z \leq 0$ indexed by $z \in \mathbb{Z}$, and define the Hilbert spaces of the past output $\mathcal{G}^{t]}$, and its future $\mathcal{G}_{t}$ as the statevector spaces of semifinite discrete strings generated by the infinite tensor products $\Phi^{t]}=\otimes_{z \leq t} \chi_{z}, \Phi_{t}=\otimes_{z>t} \chi_{z}$ with all but finite number of $\chi_{z} \in \mathfrak{g}_{z}$ equal to the initial states $\chi_{z}^{\circ}$, the copies of $\chi^{\circ}=1 \oplus 0$ in $\mathfrak{g}$ or in $\mathfrak{f}$. Denoting by $\mathcal{A}_{z}$ the copies of $\mathcal{A}_{1}=\mathcal{B}(\mathfrak{f})$ if $z>0$, and the copies of $\mathcal{A}_{0}=\mathbb{C} \oplus \mathcal{A}_{o}$ if $z \leq 0$ on the corresponding spaces $\mathfrak{g}_{z}$, we construct the local algebras $\mathcal{A}_{r}^{t]}=\otimes_{r<z \leq t} \mathcal{A}_{z}$ on $\mathfrak{g}_{r}^{t]}$, and the past (output) $\mathfrak{A}^{t]}$ and the future (input) $\mathfrak{A}_{t}$ algebras generated respectively on $\mathcal{G}^{t]}$ and on $\mathcal{G}_{t}$ by the operators $I^{t} \otimes \mathrm{A} \otimes \mathrm{I}_{s}^{t]}$ for all $s<t$, and by $\mathrm{I}_{t}^{s} \otimes \mathrm{A} \otimes I_{s}$ for all $s>t$, where $\mathrm{A} \in \mathcal{A}_{s}, \mathrm{I}_{r}^{t]}=\otimes_{r<s \leq t} \mathrm{I}_{s}, I^{t}=\mathrm{I}_{-\infty}^{t-1]}$, and $I_{t}=\otimes_{s>t} \mathrm{I}_{s}$ is the identity operator in $\mathcal{G}_{t}$. The Hilbert space $\mathcal{G}=\mathcal{G}^{0]} \otimes \mathcal{G}_{0}$, the von Neumann algebra $\mathfrak{A}=\mathfrak{A}^{0]} \otimes \mathfrak{A}_{0}$, and the product vector $\Phi=\otimes \chi_{s}^{\circ} \equiv \Phi^{\circ}$, which is an eigen-vector for any operator $A_{s}=I^{s} \otimes \mathrm{A} \otimes I_{s}$ with $s<0$ in $\mathcal{G}$, stand as candidates for the triple $(\mathcal{G}, \mathfrak{A}, \Phi)$.

Third, we define the unitary evolution on the product space $\mathcal{H}=\mathcal{G}^{0]} \otimes \mathfrak{h} \otimes \mathcal{G}_{0}$ by

$$
U: \cdots \chi_{-1} \otimes \chi_{0} \otimes \psi \otimes \chi_{1} \otimes \cdots \mapsto \cdots \chi_{0} \otimes \mathrm{U}\left(\psi \otimes \chi_{1}\right) \otimes \chi_{2} \cdots
$$

incorporating the shift in the right and left strings. They are connected by the conservative boundary condition $U: \mathfrak{h} \otimes \mathfrak{g}_{1} \rightarrow \mathfrak{g}_{0} \otimes \mathfrak{h}$ given by the unitary dilation (3.4). We have obviously

$$
\left(\left\langle\ldots, y_{-1}, y\right| \otimes I \otimes\left\langle x_{1}, \ldots\right|\right) U\left(\Phi^{0} \otimes \psi \otimes|0,0, \ldots\rangle\right)=\left\langle\ldots, y_{-1}\right| \Phi^{0} V(y) \delta_{0}^{x_{1}} \ldots
$$

in any orthonormal basis $\{|x\rangle\}$ of $\mathfrak{f}$ and $\{|y\rangle\}$ of $\mathfrak{g}$ which contain the vector $|0\rangle=$ $\chi^{\circ}$. Thus the extended unitary operator $U$ still reproduces the isometry $\mathrm{V}=$ $\sum_{y \neq 0}|y\rangle V(y)$, and

$$
\pi(\mathrm{A}, \mathrm{B})=\left(\Phi^{0]} \otimes \mathrm{I} \otimes \Phi_{0}^{\circ}\right)^{\dagger} U\left(I^{0} \otimes \mathrm{A} \otimes \mathrm{B} \otimes I_{0}\right) U^{t}\left(\Phi^{0]} \otimes \mathrm{I} \otimes \Phi_{0}^{\circ}\right)=\mathrm{V}^{\dagger}(\mathrm{A} \otimes \mathrm{B}) \mathrm{V}
$$

for all $\mathrm{A} \in \mathcal{A}_{o}$ and $\mathrm{B} \in \mathcal{B}$, where $\Phi_{0}^{\circ}=|0, \ldots\rangle$ is the vacuum vector (the infinite tensor power of vector $|0\rangle \in \mathfrak{f})$ and $\Phi^{0]}$ is arbitrary state-vector in $\mathcal{G}^{0]}$.

Fourth, we prove the dynamical invariance $U^{\dagger} \mathfrak{B} U \subseteq \mathfrak{B}$ of the algebra $\mathfrak{B}=$ $\mathfrak{A}^{0]} \otimes \mathcal{B} \otimes \mathfrak{A}_{0}$. This follows straightforward from the definition of $U$ as

$$
U^{\dagger}\left(\cdots \mathrm{A}_{0} \otimes \mathrm{B} \otimes \mathrm{A}_{1} \otimes \mathrm{A}_{2} \cdots\right) U=\cdots \mathrm{A}_{-1} \otimes \mathrm{U}^{\dagger}\left(\mathrm{A}_{0} \otimes \mathrm{B}\right) \mathrm{U} \otimes \mathrm{A}_{1} \cdots \in \mathfrak{B}
$$

due to $\mathrm{U}^{\dagger}(\mathrm{A} \otimes \mathrm{B}) \mathrm{U} \in \mathcal{B}_{1}$ for all $\mathrm{A} \in \mathcal{A}_{0}, \mathrm{~B} \in \mathcal{B}$ where $\mathcal{B}_{1}=\mathcal{B} \otimes \mathcal{A}_{1}$. However this algebra is not invariant under the inverse transformation as $\mathcal{A}_{0} \subset \mathcal{A}$, $\mathcal{B}_{0}=$ $\mathcal{A}_{0} \otimes \mathcal{B} \subset \mathcal{A}_{1} \otimes \mathcal{B}$, and if $\mathrm{U}(\mathrm{B} \otimes \mathrm{A}) \mathrm{U}^{\dagger} \notin \mathcal{B}_{0}$ for some $\mathrm{A} \in \mathcal{B}(\mathfrak{g})$ and $\mathrm{B} \in \mathcal{B}$, then

$$
U\left(\cdots \mathrm{A}_{-1} \otimes \mathrm{A}_{0} \otimes \mathrm{B} \otimes \mathrm{A}_{1} \cdots\right) U^{\dagger}=\cdots \mathrm{A}_{0} \otimes \mathrm{U}\left(\mathrm{B} \otimes \mathrm{A}_{1}\right) \mathrm{U}^{\dagger} \otimes \mathrm{A}_{2} \cdots \notin \mathfrak{B} .
$$


And fifth, we prove that the power semigroup $U^{t}, t \in \mathbb{N}$ of the constructed unitary operator induces the whole quantum Markov chain. We shall do this using the Schrödinger recurrency equation $\Psi^{t}=U \Psi^{t-1}$ with the initial condition $\Psi^{0}=$ $\Phi^{0]} \otimes \psi \otimes \Phi_{0}^{\circ}$ for $\Psi^{t}=U^{t} \Psi^{0}$ representing it as $T_{-t} \Psi(t)$, where $T_{-t}=T_{-1}^{t}$ is the right shift defined by the unitary operator

$$
T_{-1}: \cdots \chi_{0} \otimes \psi \otimes \chi_{1} \otimes \chi_{2} \cdots \mapsto \cdots \chi_{-1} \otimes \mathrm{T}^{\dagger}\left(\chi_{0} \otimes \psi\right) \otimes \chi_{1} \cdots
$$

The interaction representation evolution $\Psi(t)=U(t) \Psi^{0}$ is obviously adapted in the sense $U(t)=I^{0]} \otimes \mathrm{W}_{0}^{t]} \otimes I_{t}$, where $\mathrm{W}_{0}^{t]}$ is a unitary operator in $\mathfrak{h} \otimes \mathcal{G}_{0}^{t]}$. It can be found from the recurrency equation

$$
\mathrm{W}_{0}^{t]}=\mathrm{W}_{t}\left(\mathrm{~W}_{0}^{t-1]} \otimes \mathrm{I}\right), \quad \mathrm{W}_{0}^{0]}=\mathrm{I}
$$

as the chronological product $\mathrm{W}_{0}^{t]}=\mathrm{W}_{t}\left(\mathrm{~W}_{t-1} \otimes \mathrm{I}\right) \cdots\left(\mathrm{W}_{1} \otimes \mathrm{I}_{1}^{t]}\right)$ generated by

$$
\mathrm{W}_{t}=\mathrm{T}_{0}^{t] \dagger}\left(\mathrm{I}_{0}^{t} \otimes \mathrm{U}\right)\left(\mathrm{T}_{0}^{t-1]} \otimes \mathrm{I}\right)=\mathrm{T}_{0}^{t] \dagger}\left(I_{0}^{t} \otimes \mathrm{W}_{0}\right) \mathrm{T}_{0}^{t]},
$$

Here $\mathrm{T}_{0}^{t]}$ is the transposition of $\mathfrak{h}$ and $\mathfrak{g}_{t}$ which can also be obtained by the recurrency

$$
\mathrm{T}_{0}^{t]}=\left(\mathrm{I}_{0}^{t-1]} \otimes \mathrm{T}\right)\left(\mathrm{T}_{0}^{t-1]} \otimes \mathrm{I}\right), \quad \mathrm{T}_{0}^{0]}=\mathrm{I}
$$

and $\mathrm{W}_{0}=\mathrm{TWT}^{\dagger}=\mathrm{UT}^{\dagger}$ is the initial scattering operator obtained by transposing $\mathrm{W}=\mathrm{W}_{1}$ onto $\mathfrak{g} \otimes \mathfrak{h}$ such that $\mathrm{W}_{t}$ is applied only to the system at $s=0$ and point of the string at $s=t$ by transposing the system from the boundary $s=0$ to $t$.

Thus for the given initial condition $\Psi(0)=\Psi^{0}$ in the interaction picture we have $\Psi(t)=\Phi^{0]} \otimes \Psi_{0}(t)$, where $\Psi_{0}(t)=\psi(t) \otimes \Phi_{t}^{\circ}$ and $\psi(t)=\mathrm{W}_{0}^{t]}\left(\psi \otimes \Phi_{0}^{t]}\right)$. This $\Psi_{0}(t)=W(t) \Psi_{0}$ with $W(t)=\mathrm{W}_{0}^{t]} \otimes I_{t}$ is the solution to the quantum stochastic recurrency equation

$$
\Psi_{0}(t)=W_{t} \Psi_{0}(t-1), \quad \Psi_{0}(0)=\psi \otimes \Phi_{0}^{\circ}
$$

given by the quantum stochastic unitary generator $W_{t}=\mathrm{W}_{t} \otimes I_{t}$ in $\mathcal{H}_{0}=\mathfrak{h} \otimes \mathcal{G}_{0}$. Now we can derive the quantum filtering recurrency equation

$$
\psi\left(t, y_{0}^{t]}\right)=V\left(y_{t}\right) \psi\left(t-1, y_{0}^{t-1]}\right), \quad \psi(0)=\psi
$$

for $\psi\left(t, y_{0}^{t]}\right)=\left(\mathrm{I} \otimes\left\langle y_{0}^{t]}\right|\right) \psi(t)$ from the equation

$$
\psi(t)=\mathrm{W}_{t}\left(\psi(t-1) \otimes \chi^{\circ}\right), \quad \psi(0)=\psi
$$

for $\psi(t)=\mathrm{W}_{0}^{t]} \psi_{0}^{t]}$, where $\psi_{0}^{t]}=\psi \otimes \chi_{1}^{\circ} \otimes \ldots \otimes \chi_{t}^{\circ}$, and $\{|y\rangle\}$ is an orthonormal basis of $\mathfrak{g}$ with $|0\rangle=\chi^{\circ}=1 \oplus 0$. Indeed,

$$
\psi\left(t, y_{0}^{t]}\right)=\left(\mathrm{I} \otimes\left\langle y_{0}^{t]}\right|\right)\left(\mathrm{W}_{t} \otimes \mathrm{I}\right) \psi(t-1)=\left(\mathrm{I} \otimes\left\langle y_{t}\right|\right) \mathrm{V} \psi\left(t-1, y_{0}^{t-1]}\right)
$$

where we took into account that $\psi(t-1)=\mathrm{W}_{0}^{t-1]} \Psi_{0}^{t-1]} \otimes|0\rangle \otimes \Phi_{t}^{\circ}$ and denoted

$$
V(y) \psi=(\mathrm{I} \otimes\langle y|) \mathrm{W}_{t}(\psi \otimes|0\rangle)=(\mathrm{I} \otimes\langle y|) \mathrm{V} \psi .
$$

This proves the filtering equation in the abstract form $\psi(t)=\mathrm{V} \psi(t-1)$ which has the solution $\psi\left(t, y_{0}^{t]}\right)=V\left(t, y_{0}^{t]}\right) \psi$ corresponding to $\psi(0)=\psi$, with

$$
V\left(t, y_{0}^{t]}\right)=V\left(y^{t}\right) \cdots V\left(y^{1}\right)=\left(\left\langle y_{0}^{t]}\right| \otimes \mathrm{I}\right) \mathrm{V}(t)
$$


Moreover, if $\mathrm{A}_{0}^{t]}=\left(\mathrm{A}^{1}, \ldots, \mathrm{A}^{t}\right)$ is any sequence of the operators $\mathrm{A}^{r} \in \mathcal{A}_{o}$, then obviously

$$
\begin{aligned}
\pi\left(t, \mathrm{~A}_{0}^{t]}, \mathrm{B}\right) & =(\mathrm{I} \otimes\langle 0, \ldots, 0|) \mathrm{W}_{0}^{t] \dagger}\left(\mathrm{B} \otimes \mathrm{A}^{1} \otimes \cdots \otimes \mathrm{A}^{t}\right) \mathrm{W}^{t]}(\mathrm{I} \otimes|0, \ldots, 0\rangle) \\
& =U^{-t}\left(I^{-t]} \otimes \mathrm{A}^{1} \otimes \cdots \otimes \mathrm{A}^{t} \otimes \mathrm{B} \otimes I_{0}\right) U^{t} \in \mathcal{B}, \quad \forall \mathrm{B} \in \mathcal{B} .
\end{aligned}
$$

This gives a dynamical endomorphic realization of any quantum Markov chain $\pi(t)$ induced by a unitary semigroup $U^{t}$.

Note that we also proved that any stochastic $\mathcal{B}$-Markov chain has a quantum stochastic realization $W(t)=\mathrm{W}_{0}^{t]} \otimes I_{t}$ given by a unitary generator $\mathrm{W}$ in $\mathfrak{h} \otimes \mathfrak{g}$ which belongs to $\mathcal{B} \otimes \mathcal{B}(\mathfrak{g})$ as commuting with $\mathcal{B}^{\prime} \otimes \mathrm{I}$. It is simply the interaction evolution induced on $\mathcal{H}_{0}=\mathfrak{h} \otimes \mathcal{G}_{0}$ by $U(t)=I^{0} \otimes W(t)$ for the positive $t \in \mathbb{N}$. It defines an adapted quantum stochastic cocycle of authomorphisms $\omega\left(t, B_{0}\right)=W(t)^{\dagger} B_{0} W(t)$ on the algebra $\mathfrak{B}_{0}=\mathcal{B} \otimes \mathfrak{A}_{0}$ as a family of "system $\mathcal{B}$ plus noise $\mathfrak{A}_{0}$ " adapted representations

$$
\omega\left(t, \mathrm{~B} \otimes \mathrm{A}_{0}^{t]} \otimes A_{t}\right)=\mathrm{W}_{0}^{t] \dagger}\left(\mathrm{B} \otimes \mathrm{A}_{0}^{t]}\right) \mathrm{W}_{0}^{t]} \otimes A_{t} \quad \forall A_{t} \in \mathcal{B}\left(\mathcal{G}_{t}\right)
$$

of this algebra, where $A_{0}^{t]} \in \mathcal{B}\left(\mathfrak{g}_{0}^{t]}\right)$. Their compositions $\vartheta^{t}=\omega(t) \circ \tau_{-t}$ with the right shifts $\tau_{-t}\left(B_{-t}\right)=T_{t}\left(I^{t} \otimes B_{[-t}\right) T_{-t}$ of $B_{-t} \in \mathfrak{B}_{-t}$ into $\mathfrak{B}_{0}$ obviously extends to the semigroup of injective endomorphisms

$$
U^{-t}\left(A^{-t]} \otimes \mathrm{A}_{-t}^{0]} \otimes \mathrm{B} \otimes A_{0}\right) U^{t}=A^{0]} \otimes \mathrm{U}_{0}^{t] \dagger}\left(\mathrm{A}_{-t}^{0]} \otimes \mathrm{B}\right) \mathrm{U}_{0}^{t]} \otimes A_{t}
$$

on the algebra $\mathfrak{B}$, where $\mathrm{U}_{0}^{t]}=\mathrm{T}_{0}^{t]} \mathrm{W}_{0}^{t]}$ are given by the same recurrency as $\mathrm{T}_{0}^{t]}$ but with the generator $\mathrm{U}$ instead of $\mathrm{T}$. This semigroup in general is not invertible on $\mathcal{B}$ if $\mathcal{A}_{0}$ is smaller then $\mathcal{A}_{1}$ (the shifts $\tau_{t}$ are not invertible in this case) despite the invertibility of the interaction evolution described by the authomorphisms $\omega(t)$.

\subsection{Symbolic Quantum Calculus and Stochastic Differential Equations.} In order to formulate the differential nondemolition causality condition and to derive a filtering equation for the posterior states in the time-continuous case we need quantum stochastic calculus.

The classical differential calculus for the infinitesimal increments

$$
\mathrm{d} x=x(t+\mathrm{d} t)-x(t)
$$

became generally accepted only after Newton gave a simple algebraic rule $(\mathrm{d} t)^{2}=0$ for the formal computations of the differentials $\mathrm{d} x$ for smooth trajectories $t \mapsto x(t)$. In the complex plane $\mathbb{C}$ of phase space it can be represented by a one-dimensional algebra $\alpha=\mathbb{C d}_{t}$ of the elements $a=\alpha \mathrm{d}_{t}$ with involution $a^{\star}=\bar{\alpha} \mathrm{d}_{t}$. Here

$$
\mathrm{d}_{t}=\left[\begin{array}{ll}
0 & 1 \\
0 & 0
\end{array}\right]=\frac{1}{2}\left(\sigma_{x}+i \sigma_{y}\right)
$$

for $\mathrm{d} t$ is the nilpotent matrix, which can be regarded as Hermitian $\mathrm{d}_{t}^{\star}=\mathrm{d}_{t}$ with respect to the Minkowski metrics $(\mathbf{z} \mid \mathbf{z})=2 \operatorname{Re} z_{-} \bar{z}_{+}$in $\mathbb{C}^{2}$.

This formal rule was generalized to non-smooth paths early in the last century in order to include the calculus of forward differentials $\mathrm{d} w \simeq(\mathrm{d} t)^{1 / 2}$ for continuous diffusions $w_{t}$ which have no derivative at any $t$, and the forward differentials $\mathrm{d} n \in$ $\{0,1\}$ for left continuous counting trajectories $n_{t}$ which have zero derivative for 
almost all $t$ (except the points of discontinuity where $\mathrm{d} n=1$ ). The first is usually done by adding the rules

$$
(\mathrm{d} w)^{2}=\mathrm{d} t, \quad \mathrm{~d} w \mathrm{~d} t=0=\mathrm{d} t \mathrm{~d} w
$$

in formal computations of continuous trajectories having the first order forward differentials $\mathrm{d} x=\alpha \mathrm{d} t+\beta \mathrm{d} w$ with the diffusive part given by the increments of standard Brownian paths $w$. The second can be done by adding the rules

$$
(\mathrm{d} n)^{2}=\mathrm{d} n, \quad \mathrm{~d} n \mathrm{~d} t=0=\mathrm{d} t \mathrm{~d} n
$$

in formal computations of left continuous and smooth for almost all $t$ trajectories having the forward differentials $\mathrm{d} x=\alpha \mathrm{d} t+\gamma \mathrm{d} m$ with jumping part given by the increments of standard compensated Poisson paths $m_{t}=n_{t}-t$. These rules were developed by Itô [110] into the form of a stochastic calculus.

The linear span of $\mathrm{d} t$ and $\mathrm{d} w$ forms the Wiener-Itô algebra $\mathfrak{b}=\mathbb{C d}_{t}+\mathbb{C d}_{w}$, while the linear span of $\mathrm{d} t$ and $\mathrm{d} n$ forms the Poisson-Itô algebra $\mathfrak{c}=\mathbb{C} \mathrm{d}_{t}+\mathbb{C d}_{m}$, with the second order nilpotent $\mathrm{d}_{w}=\mathrm{d}_{w}^{\star}$ and the idempotent $\mathrm{d}_{m}=\mathrm{d}_{m}^{\star}$. They are represented together with $\mathrm{d}_{t}$ by the triangular Hermitian matrices

$$
\mathrm{d}_{t}=\left[\begin{array}{lll}
0 & 0 & 1 \\
0 & 0 & 0 \\
0 & 0 & 0
\end{array}\right], \quad \mathrm{d}_{w}=\left[\begin{array}{lll}
0 & 1 & 0 \\
0 & 0 & 1 \\
0 & 0 & 0
\end{array}\right], \quad \mathrm{d}_{m}=\left[\begin{array}{ccc}
0 & 1 & 0 \\
0 & 1 & 1 \\
0 & 0 & 0
\end{array}\right],
$$

on the Minkowski space $\mathbb{C}^{3}$ with respect to the inner Minkowski product $(\mathbf{z} \mid \mathbf{z})=$ $z_{-} z^{-}+z_{\circ} z^{\circ}+z_{+} z^{+}$, where $z^{\mu}=\bar{z}_{-\mu},-(-, \circ,+)=(+, \circ,-)$.

Although both algebras $\mathfrak{b}$ and $\mathfrak{c}$ are commutative, the matrix algebra $\mathfrak{a}$ generated by $\mathfrak{b}$ and $\mathfrak{c}$ on $\mathbb{C}^{3}$ is not:

$$
\mathrm{d}_{w} \mathrm{~d}_{m}=\left[\begin{array}{ccc}
0 & 1 & 1 \\
0 & 0 & 0 \\
0 & 0 & 0
\end{array}\right] \neq\left[\begin{array}{ccc}
0 & 0 & 1 \\
0 & 0 & 1 \\
0 & 0 & 0
\end{array}\right]=\mathrm{d}_{m} \mathrm{~d}_{w}
$$

The four-dimensional $\star$-algebra $\mathfrak{a}=\mathbb{C d}_{t}+\mathbb{C d}_{-}+\mathbb{C d}^{+}+\mathbb{C d}$ of triangular matrices with the canonical basis

$$
\mathrm{d}_{-}=\left[\begin{array}{lll}
0 & 1 & 0 \\
0 & 0 & 0 \\
0 & 0 & 0
\end{array}\right], \mathrm{d}^{+}=\left[\begin{array}{ccc}
0 & 0 & 0 \\
0 & 0 & 1 \\
0 & 0 & 0
\end{array}\right], \mathrm{d}=\left[\begin{array}{lll}
0 & 0 & 0 \\
0 & 1 & 0 \\
0 & 0 & 0
\end{array}\right],
$$

given by the algebraic combinations

$$
\mathrm{d}_{-}=\mathrm{d}_{w} \mathrm{~d}_{m}-\mathrm{d}_{t}, \mathrm{~d}^{+}=\mathrm{d}_{m} \mathrm{~d}_{w}-\mathrm{d}_{t}, \mathrm{~d}=\mathrm{d}_{m}-\mathrm{d}_{w}
$$

is the canonical representation of the differential $\star$-algebra for one-dimensional vacuum noise in the unified quantum stochastic calculus [79, 83]. It realizes the HP (Hudson-Parthasarathy) table 77

$$
\mathrm{d} A_{-} \mathrm{d} A^{+}=\mathrm{d} t, \quad \mathrm{~d} A_{-} \mathrm{d} A=\mathrm{d} A_{-}, \quad \mathrm{d} A \mathrm{~d} A^{+}=\mathrm{d} A^{+}, \quad(\mathrm{d} A)^{2}=\mathrm{d} A,
$$

with zero products for all other pairs, for the multiplication of the canonical counting $\mathrm{d} A=\lambda(\mathrm{d})$, creation $\mathrm{d} A^{+}=\lambda\left(\mathrm{d}^{+}\right)$, annihilation $\mathrm{d} A_{-}=\lambda\left(\mathrm{d}_{-}\right)$, and preservation $\mathrm{d} t=\lambda\left(\mathrm{d}_{t}\right)$ quantum stochastic integrators in Fock space over $L^{2}\left(\mathbb{R}_{+}\right)$. As was proved recently in [111], any generalized Itô algebra describing a quantum noise can be represented in the canonical way as a $\star$-subalgebra of a quantum vacuum algebra

$$
\mathrm{d} A_{\mu}^{\kappa} \mathrm{d} A_{\iota}^{\nu}=\delta_{\iota}^{\kappa} \mathrm{d} A_{\mu}^{\nu}, \quad \iota, \mu \in\{-, 1, \ldots, d\} ; \kappa, \nu \in\{1, \ldots, d,+\}
$$


in the Fock space with several degrees of freedom $d$, where $\mathrm{d} A_{-}^{+}=\mathrm{d} t$ and $d$ is restricted by the doubled dimensionality of quantum noise (could be infinite), similar to the representation of every semi-classical system with a given state as a subsystem of quantum system with a pure state. Note that in this quantum Itô product formula $\delta_{\kappa}^{\iota}=0$ if $\iota=+$ or $\kappa=-$ as $\delta_{\kappa}^{\iota} \neq 0$ only when $\iota=\kappa$.

The quantum Itô product gives an explicit form

$$
\mathrm{d} \psi \psi^{\dagger}+\psi \mathrm{d} \psi^{\dagger}+\mathrm{d} \psi \mathrm{d} \psi^{\dagger}=\left(\alpha_{\kappa}^{\iota} \psi^{\dagger}+\psi \alpha_{\kappa}^{\star \iota}+\alpha_{j}^{\iota} a_{\kappa}^{\star j}\right)_{\kappa}^{\iota} \mathrm{d} A_{\iota}^{\kappa}
$$

of the term $\mathrm{d} \psi \mathrm{d} \psi^{\dagger}$ for the adjoint quantum stochastic differentials

$$
\mathrm{d} \psi=\alpha_{\kappa}^{\iota} \mathrm{d} A_{\iota}^{\kappa}, \quad \mathrm{d} \psi^{\dagger}=\alpha_{\kappa}^{\star \iota} \mathrm{d} A_{\iota}^{\kappa},
$$

for evaluation of the product differential

$$
\mathrm{d}\left(\psi \psi^{\dagger}\right)=(\psi+\mathrm{d} \psi)(\psi+\mathrm{d} \psi)^{\dagger}-\psi \psi^{\dagger}
$$

Here $\alpha_{-\kappa}^{\star \iota}=\alpha_{-\iota}^{\kappa \dagger}$ is the quantum Itô involution with respect to the switch $-(-,+)=$ $(+,-),-(1, \ldots, d)=(1, \ldots, d)$, introduced in [79], and the Einstein summation is always understood over $\kappa=1, \ldots, d,+; \iota=-, 1, \ldots, d$ and $k=1, \ldots, d$. This is the universal Itô product formula which lies in the heart of the general quantum stochastic calculus [79, 83] unifying the Itô classical stochastic calculi with respect to the Wiener and Poisson noises and the quantum differential calculi 77, 78 based on the particular types of quantum Itô algebras for the vacuum or finite temperature noises. It was also extended to the form of quantum functional Itô formula and even for the quantum nonadapted case in [112, 113.

Every stationary classical (real or complex) process $x^{t}, t>0$ with $x^{0}=0$ and independent increments $x^{t+\Delta}-x^{t}$ has mean values $\mathrm{M}\left[x^{t}\right]=\lambda t$. The compensated process $y^{t}=x^{t}-\lambda t$, which is called noise, has an operator representation $\hat{x}^{t}$ in Fock space $\mathcal{F}_{0}$ the Hilbert space $L^{2}\left(\mathbb{R}_{+}\right)$in the form of the integral with respect to basic processes $A_{j}^{+}, A_{-}^{j}, A_{k}^{i}$ such that $\digamma=f(\hat{x}) \delta_{\varnothing} \simeq f(x)$ in terms of the $L_{\mu}^{2}-$ Fock isomorphism $f \longleftrightarrow \digamma$ of the chaos expansions

$$
f(x)=\sum_{n=0}^{\infty} \int \cdots \int_{0<r_{1}<\ldots<r_{n}} \digamma\left(r_{1}, \ldots r_{n}\right) \mathrm{d} y^{r_{1}} \cdots \mathrm{d} y^{r_{n}} \equiv \int \digamma(v) \mathrm{d} y^{v}
$$

of the stochastic functionals $f \in L_{\mu}^{2}$ having the finite second moments $\mathrm{M}\left[|f|^{2}\right]=$ $\|\digamma\|^{2}$ and the Fock vectors $\digamma \in \mathcal{F}_{0}$. The expectations of the Fock operators $f(\hat{x})$ given by the iterated stochastic integrals $f$ coincides on the vacuum state-vector $\delta_{\varnothing} \in \mathcal{F}_{0}$ with their expectation given by the probability measure $\mu$ :

$$
\mathrm{M}[f(x)]=\left\langle\delta_{\varnothing} \mid f(\hat{x}) \delta_{\varnothing}\right\rangle=\digamma(\varnothing) .
$$

If its differential increments $\mathrm{d} x^{t}$ form a two dimensional Itô algebra, $\hat{x}^{t}$ can be represented in the form of a commutative combination of the three basic quantum stochastic increments $A=A_{0}^{0}, A_{-}=A_{-}^{0}, A^{+}=A_{0}^{+}$. The Itô formula for the process $x^{t}$ given by the quantum stochastic differential

$$
\mathrm{d} \hat{x}^{t}=\alpha \mathrm{d} A+\alpha^{-} \mathrm{d} A_{-}+\alpha_{+} \mathrm{d} A^{+} \mathrm{d} \psi+\alpha_{+}^{-} \mathrm{d} t
$$

can be obtained from the HP product 77

$$
\mathrm{d} \hat{x}^{t} \mathrm{~d} \hat{x}^{t \dagger}=\alpha \alpha^{\dagger} \mathrm{d} A+\alpha^{-} \alpha^{\dagger} \mathrm{d} A_{-}+\alpha \alpha^{-\dagger} \mathrm{d} A^{+}+\alpha^{-} \alpha^{-\dagger} \mathrm{d} t .
$$


The noises $y_{k}^{t}=x_{k}^{t}-\lambda_{k} t$ with stationary independent increments are called standard if they have the standard variance $\mathrm{M}\left[\left(x^{t}\right)^{2}\right]=t$. In this case

$$
\hat{y}_{k}^{t}=\left(A_{k}^{+}+A_{-}^{k}+\varepsilon_{k} A_{k}^{k}\right)(t)=\varepsilon_{k} m_{k}^{t}+\left(1-\varepsilon_{k}\right) w_{k}^{t},
$$

where $\varepsilon_{k} \geq 0$ is defined by the equation $\left(\mathrm{d} x_{k}^{t}\right)^{2}-\mathrm{d} t=\varepsilon \mathrm{d} x_{k}^{t}$. Such, and indeed higher dimensional, quantum noises for continual measurements in quantum optics were considered in 114, 115.

The general form of a quantum stochastic decoherence equation, based on the canonical representation of the arbitrary Itô algebra for a quantum noise in the vacuum of $d$ degrees of freedom, can be written as

$$
\mathrm{d} \hat{\psi}(t)=\left(\mathrm{S}_{\kappa}^{\iota}-\delta_{\kappa}^{\iota} \mathrm{I}\right) \mathrm{d} A_{\iota}^{\kappa} \hat{\psi}(t), \quad \hat{\psi}(0)=\psi \otimes \delta_{\varnothing}, \psi \in \mathfrak{h} .
$$

Here $\mathrm{L}_{\kappa}^{\iota}$ are the operators in the system Hilbert space $\mathfrak{h} \ni \psi$ with $\mathrm{S}_{\kappa}^{\star-} \mathrm{S}_{+}^{\kappa}=0$ for the mean square normalization

$$
\hat{\psi}(t)^{\dagger} \hat{\psi}(t)=\mathrm{M}\left[\psi(t, \cdot)^{\dagger} \psi(t, \cdot)\right]=\psi^{\dagger} \psi
$$

with respect to the vacuum of Fock space of the quantum noise, where the Einstein summation is understood over all $\kappa=-, 1, \ldots, d,+$ with the agreement

$$
\mathrm{S}_{-}^{-}=\mathrm{I}=\mathrm{S}_{+}^{+}, \quad \mathrm{S}_{-}^{j}=\mathrm{O}=\mathrm{S}_{j}^{+}, \quad j=1, \ldots, d
$$

and $\delta_{\kappa}^{\iota}=1$ for all coinciding $\iota, \kappa \in\{-, 1, \ldots, d,+\}$ such that $\mathrm{L}_{\kappa}^{\iota}=\mathrm{S}_{\kappa}^{\iota}-\delta_{\kappa}^{\iota} \mathrm{I}=0$ whenever $\iota=+$ or $\kappa=-$. In the notations $\mathrm{S}_{+}^{j}=\mathrm{L}^{j}, \mathrm{~S}_{+}^{-}=-\mathrm{K}, \mathrm{S}_{j}^{-}=-\mathrm{K}_{j}$, $j=1, \ldots, d$ the decoherence wave equation takes the standard form 97, 117]

$$
\mathrm{d} \hat{\psi}(t)+\left(\mathrm{Kd} t+\mathrm{K}_{j} \mathrm{~d} A_{-}^{j}\right) \hat{\psi}(t)=\left(\mathrm{L}^{j} \mathrm{~d} A_{j}^{+}+\left(\mathrm{S}_{k}^{i}-\delta_{k}^{i} \mathrm{I}\right) \mathrm{d} A_{i}^{k}\right) \hat{\psi}(t),
$$

where $A_{j}^{+}(t), A_{-}^{j}(t), A_{i}^{k}(t)$ are the canonical creation, annihilation and exchange processes respectively in Fock space, and the normalization condition is written as $\mathrm{L}_{k} \mathrm{~L}^{k}=\mathrm{K}+\mathrm{K}^{\dagger}$ with $\mathrm{L}_{k}^{\dagger}=\mathrm{L}^{k}$ (the Einstein summation is over $i, j, k=1, \ldots, d$ ).

Using the quantum Itô formula one can obtain the corresponding equation for the quantum stochastic density operator $\hat{\varrho}=\psi \psi^{\dagger}$ which is the particular case $\kappa=-, 1, \ldots, d,+$ of the general quantum stochastic Master equation

$$
\mathrm{d} \hat{\varrho}(t)=\left(\mathrm{S}_{\gamma}^{\iota} \hat{\varrho}(t) \mathrm{S}_{\kappa}^{\star \gamma}-\hat{\varrho}(t) \delta_{\kappa}^{\iota}\right) \mathrm{d} A_{\iota}^{\kappa}, \quad \hat{\varrho}(0)=\rho,
$$

where the summation over $\kappa=-, k,+$ is extended to infinite number of $k=1,2, \ldots$. This general form of the decoherence equation with $\mathrm{L}_{\kappa}^{\star-} \mathrm{L}_{+}^{\kappa}=\mathrm{O}$ corresponding to the normalization condition $\langle\hat{\varrho}(t)\rangle=\operatorname{Tr} \rho$ in the vacuum mean, was recently derived in terms of quantum stochastic completely positive maps in [97, 117. Denoting $\mathrm{L}_{\kappa}^{-}=-\mathrm{K}_{\kappa}, \mathrm{L}_{+}^{\star \iota}=-\mathrm{K}^{\iota}$ such that $\mathrm{K}_{\iota}^{\dagger}=\mathrm{K}^{\iota}$, this can be written as

$$
\mathrm{d} \varrho(t)+\mathrm{K}_{\kappa} \hat{\varrho}(t) \mathrm{d} A_{-}^{\kappa}+\hat{\varrho}(t) \mathrm{K}^{\iota} \mathrm{d} A_{\iota}^{+}=\left(\mathrm{L}_{\kappa}^{j} \hat{\varrho}(t) \mathrm{L}_{j}^{\star \iota}-\hat{\varrho}(t) \delta_{\kappa}^{\iota}\right) \mathrm{d} A_{\iota}^{\kappa},
$$

or in the notation above, $\mathrm{K}_{+}=\mathrm{K}, \mathrm{K}^{-}=\mathrm{K}^{\dagger}, \mathrm{L}_{+}^{k}=\mathrm{L}^{k}, \mathrm{~L}_{k}^{\star-}=\mathrm{L}_{k}, \mathrm{~L}_{k}^{\star i}=\mathrm{L}_{i}^{k \dagger}$ as

$$
\begin{aligned}
\mathrm{d} \varrho(t) & +\left(\mathrm{K} \varrho(t)+\hat{\varrho}(t) \mathrm{K}^{\dagger}-\mathrm{L}^{j} \hat{\varrho}(t) \mathrm{L}_{j}\right) \mathrm{d} t=\left(\mathrm{S}_{k}^{j} \hat{\varrho}(t) \mathrm{S}_{j}^{\dagger i}-\hat{\varrho}(t) \delta_{k}^{i}\right) \mathrm{d} A_{i}^{k} \\
& +\left(\mathrm{S}_{k}^{j} \hat{\varrho}(t) \mathrm{L}_{j}-\mathrm{K}_{k} \hat{\varrho}(t)\right) \mathrm{d} A_{-}^{k}+\left(\mathrm{L}^{j} \hat{\varrho}(t) \mathrm{S}_{j}^{\dagger i}-\hat{\varrho}(t) \mathrm{K}^{i}\right) \mathrm{d} A_{i}^{+},
\end{aligned}
$$

with $\mathrm{K}+\mathrm{K}^{\dagger}=\mathrm{L}_{j} \mathrm{~L}^{j}, \mathrm{~L}^{j}=\mathrm{L}_{j}^{\dagger}, \mathrm{L}_{k}^{\dagger i}=\mathrm{L}_{i}^{k \dagger}$ for any number of $j$ 's, and arbitrary $\mathrm{K}^{j}=\mathrm{K}_{j}^{\dagger}, \mathrm{L}_{k}^{i}, i, j, k=1, \ldots, d$. This is the quantum stochastic generalization of 
the general form [118] for the non-stochastic (Lindblad) Master equation corresponding to the case $d=0$. In the case $d>0$ with pseudo-unitary block-matrix $\mathrm{S}=\left[\mathrm{S}_{\kappa}^{\iota}\right]_{\nu=-, 0,+}^{\iota=-, o,+}$ in the sense $\mathbf{S}^{\star}=\mathbf{S}^{-1}$, it gives the general form of quantum stochastic Langevin equation corresponding to the HP unitary evolution for $\psi(t)$ [77].

The nonlinear form of this decoherence equation for the exactly normalized density operator $\hat{\rho}(t)=\hat{\varrho}(t) / \operatorname{Tr}_{\mathfrak{h}} \hat{\varrho}(t)$ was obtained for different commutative Itô algebras in [13, 91, 83].

\section{Acknowledgment:}

I would like to acknowledge the help of Robin Hudson and some of my students attending the lecture course on Modern Quantum Theory who were the first who read and commented on these notes containing the answers on some of their questions. The best source on history and drama of quantum theory is in the biographies of the great inventors, Schrödinger, Bohr and Heisenberg [55, 119, 120], and on the conceptual development of this theory before the rise of quantum probability - in 121. An excellent essay "The quantum age begins", as well as short biographies with posters and famous quotations of all mathematicians and physicists mentioned here can be found on the mathematics website at St Andrews University http://www-history.mcs.st-and.ac.uk/history/, the use of which is acknowledged.

\section{REFERENCES}

[1] J. S. Bell, Speakable and Unspeakable in Quantum Mechanics. Camb. Univ. Press, 1987.

[2] V.P. Belavkin, Nondemolition Principle of Quantum Measurement Theory. Foundations of Physics, 24, No. 5, 685-714 (1994).

[3] V. P. Belavkin, Optimal Measurement and Control in Quantum Dynamical Systems. Preprint No. 411, Inst. of Phys.., Nicolaus Copernicus University, Torun', February 1979. Published in Rep. Math. Phys., 43, No. 3, 405-425, (1999).

[4] V. P. Belavkin, Quantum Filtering of Markov Signals with Wight Quantum Noise. Radiotechnika and Electronika, 25, 1445-1453 (1980). Full English translation in: Quantum Communications and Measurement. V. P. Belavkin et al, eds., 381-392 (Plenum Press, 1994).

[5] V. P. Belavkin, In: Information Complexity and Control in Quantum Physics, ed. A. Blaquière, 331-336 (Springer-Verlag, Udine 1985).

[6] A. Barchielli \& G. Lupierri. J Math Phys., 26, 2222-30 (1985).

[7] V. P. Belavkin, In: Modelling and Control of Systems, ed. A. Blaquière, Lecture Notes in Control and Information Sciences, 121, 245-265, Springer 1988.

[8] L. Diosi, Phys. Rev. A, 40, 1165-74 (1988).

[9] V. P. Belavkin, A Continuous Counting Observation and Posterior Quantum Dynamics. J. Phys. A: Math. Gen. 22, L1109-L1114 (1989).

[10] N. Gisin. Helv. Phys. Acta, 62, 363 (1989).

[11] V. P. Belavkin, In: Stochastic Methods in Experimental Sciences, W. Kasprzak and A. Weron eds., 26-42 (World Scientific, 1990); A Posterior Schrödinger Equation for Continuous Nondemolition Measurement. J. Math. Phys. 31, 2930-2934 (1990).

[12] G. C. Ghirardi, P. Pearl \& A. Rimini, Markov Processes in Hilbert Space and Continuous Spontaneous Localization of Systems of Identical Particles. Phys. Rev. A 42, 78-89 (1990).

[13] V. P. Belavkin, Stochastic Posterior Equations for Quantum Nonlinear Filtering. In: Prob. Theory and Math. Stat., ed. B Grigelionis et al., 1, 91-109, VSP/Mokslas, Vilnius 1990.

[14] E. Wigner. Am. J. Phys. 31, 6 (1963).

[15] E. B. Davies, Quantum Theory of Open Systems. Academic, 1976.

[16] V. P. Belavkin, Operational theory of quantum stochastic processes. In: Proc. of VII-th Conference on Coding Theory and Information transmission, 1, 23-28 (Moscow-Vilnius, 1978).

[17] A. Barchielli, L. Lanz \& G.M. Prosperi. Nuovo Cimento, 72B, 79 (1982). 
[18] A. S. Holevo. Soviet Mathematics 26, 1-20 (1982); Izvestia Vuzov, Matematica, 26, 3-19 (1992).

[19] V. P. Belavkin, Towards Control Theory of Quantum Observable Systems. Automatica and Remote Control, 44,178-188 (1983).

[20] A. Barchielli. Nuovo Cimento, 74B, 113-138 (1983).

[21] H. D. Zeh. Foundation of Physics. 1, 69 (1970).

[22] W. G. Unruh \& W. G. Zurek. Physical Review D, 40, 1071 (1989).

[23] P. Pearle, Reduction of the State Vector by a Nonlinear Schrödinger Equation. Phys. Rev. D, 13, No. 4, 857-868 (1976).

[24] N. Gisin. J. Math. Phys., 24, 1779-82 (1983).

[25] M. Gell-Mann \& J. B. Hartle. In: Complexity, Entropy and Physics of Information, W. H. Zurek, ed. (Addison-Wesley, 1990).

[26] R. Haag, An evolutionary picture for quantum physics. Com. Math. Phys., 180, 733-743 (1995).

[27] G. C. Ghirardi, A. Rimini \& T. Weber. Phys. Rev. D 34, No 2, 470-491 (1986).

[28] Ph. Blanchard \& A. Jadczyk, Event-Enhanced-Quantum Theory and Piecewise deterministic Dynamics. Annalen der Physik, 4, 583-599 (1995).

[29] M. B. Mensky, Continuous Quantum Measurements and Paths Integrals, IOP, Bristol 1993.

[30] S. Albeverio, V. N. Kolokol'tsov \& O. G. Smolyanov, Continuous Quantum Measurement: Local and Global Approaches. Rew. Math. Phys., 9, 907-920 (1997).

[31] G. J. Milburn \& D. E. Walls. Phys. Rev., 30(A), 56-60 (1984).

[32] D. F. Walls, Collet M.J., and Milburn G.J. Phys. Rev., 32(D),3208-15 (1985).

[33] H. Carmichael, Open Systems in Quantum Optics. Lecture Notes in Physics, m18, (SpringerVerlag, 1986).

[34] P. Zoller, M. A. Marte \& D. F. Walls. Phys. Rev., 35(A), 198-207 (1987).

[35] A. Barchielli. J. Phys. A: Math. Gen., 20, 6341-55 (1987).

[36] C. A. Holmes, G. J. Milburn \& D. F. Walls. Phys. Rev., 39(A), 2493-501 (1989).

[37] M. Ueda. Phys. Rev., 41(A), 3875-90 (1990).

[38] G. Milburn \& Gagen. Phys Rev. A, 46,1578 (1992).

[39] N. Gisin, Quantum Measurement and Stochastic Processes. Phys. Rev. Lett., 52, No. 19, 1657-60 (1984).

[40] N. Gisin \& I. C. Percival, The Quantum State Diffusion Model Applied to Open systems. J. Phys. A: Math. Gen. 25, 5677-91 (1992).

[41] N. Gisin \& I. C. Percival, The Quantum State Diffusion Picture of Physical Processes. J. Phys. A: Math. Gen. 26, 2245-60 (1993).

[42] H. J. Carmichael, An Open System Approach to Quantum Optics, Lecture Notes in Physics, m18 (Springer, Berlin, 1993).

[43] H. M. Wiseman \& G. J. Milburn. Phys. Rev. A 47, 642 (1993).

[44] P. Goetsch \& R. Graham, Quantum Trajectories for Nonlinear Optical Processes. Ann. Physik 2, 708-719 (1993).

[45] H. M. Wiseman \& G. J. Milburn. Phys. Rev. A 49, 1350 (1994).

[46] H. J. Carmichael. In: Quantum Optics VI, ed J. D. Harvey and D. F. Walls (Springer, Berlin, 1994).

[47] P. Goetsch \& R. Graham, Linear Stochastic Wave Equation for Continuously Measurement Quantum Systems. Phys. Rev. A 50, 5242-55 (1994).

[48] P. Goetsch, R. Graham, \& F. Haake, Schrödinger Cat and Single Runs for the Damped Harmonic Oscillator. Phys. Rev. A, 51, No. 1, 136-142 (1995).

[49] M. Planck, Scientific Autobiography, and Other Papers. Williams \& Norgate LTD. London 1949.

[50] V. P. Belavkin. Quantum Noise, Bits and Jumps. Progress in quantum Electronics, 25, No. 1, 1-53 (2001).

[51] W. Heisenberg. Z. Phys. 33, 879-93 (1925).

[52] M. Born, W. Heisenberg \& P. Z. Jordan, Phys. 36, 557-615 (1926).

[53] E. Schrödinger, Quantization as an Eigenvalue Problem. Ann. Phys. 79, 361-76 (1926).

[54] E. Schrödinger, Abhandlundgen zur Wellenmechanik. Leipzig: J.A. Barth (1926).

[55] W. Moore, Schrödinger life and thought. Cambridge University Press (1989).

[56] D. Bohm. Phys. Rev. 85, 166, 180 (1952).

[57] H. Everett, Rev. Mod. Phys. 29, 454 (1957). 
V P BELAVKIN

[58] W. Heisenberg, On the Perceptual Content of Quantum Theoretical Kinematics and Mechanics. Z. Phys. 43, 172-198 (1925). English translation in: J. A. Wheeler and Wojciech Zurek, eds. Quantum Theory and Measurement. (Princeton University Press, 1983), pp. $62-84$.

[59] Y. Aharonov \& D. Bohm, Phys. Rev. 122, 1649 (1961).

[60] V. P. Belavkin, Generalized Uncertaity Relations and Efficient Measurements in Quantum Systems. Theoretical and Mathematical Physics, 26, No 3, 316-329 (1976), The Nondemolition Measurement of Quantum Time. International Journal of Theoretical Physics, 37, No1, 219-226 (1998).

[61] A. S. Holevo, Probabilistic Aspects of Quantum Theory, Kluwer Publisher, 1980.

[62] V. P. Belavkin \& M. G. Perkins, Nondemolition Measurement of Quantum Time. Int. J. of Theor. Phys. 37, No 1 219-226 (1998).

[63] J. von Neumann, Mathematische Grundlagen der Quantummechanik. Springer, Berlin, 1932. (English translation : Prinston University Press, 1955).

[64] J. S. Bell, On the Problem of Hidden Variables in Quantum Theory. Rev. Mod. Phys., 38, 447-452 (1966).

[65] A. M. Gleason. J. Math. \& Mech., 6, 885 (1957).

[66] G. Birkhoff \& J. von Neumann, The Logic of Quantum Mechanics. Annals of Mathematics 37, 823-843 (1936).

[67] G. C. Wick, A. S. Wightman \& E. P. Wigner, The Intrinsic Parity of Elementary Particles. Phys. Rev. 88, 101-105 (1952).

[68] J. P. Jauch \& G. Piron, Can Hidden Variables be Excluded in Quantum mechanics? Helv. Phys. Acta 36, 837 (1963).

[69] B. Russel, Mysticism and Logic, p.75. Penguin, London (1953).

[70] A. Einstein, B. Podolski \& N. Rosen, Can Quantum-Mechanical Description of Physical Reality be Considered Complete? Phys.Rev. 47, 777-800 (1935).

[71] N. Bohr, Phys. Rev. 48, 696-702 (1935).

[72] E. Schrödinger, Naturwis. 23, 807-12, 823-8, 844-9 (1935).

[73] G. Lüders, Ann. Phys. (Leipzig) 8, 322 (1951).

[74] P. Bush, P. J. Lahti, P. Mittelstädt, The Quantum Measurement Theory. Lecture Notes in Physics, Springer-Verlag, Berlin, 1991.

[75] R. L. Stratonovich \& V. P. Belavkin, Dynamical Interpretation for the Quantum Measurement Projection Postulate. Int. J. of Theor. Phys., 35, No. 11, 2215-2228 (1996).

[76] C. W. Gardiner, Quantum Noise. Springer-Verlag, Berlin Heidelberg 1991.

[77] R. L. Hudson \& K. R. Parthasarathy, Quantum Itô's Formula and Stochastic Evolution. Comm. Math. Phys., 93, 301-323 (1984).

[78] C. W. Gardiner \& M. J. Collett. Phys. Rev. A, 31, 3761 (1985).

[79] V. P. Belavkin, A New Form and $a \star$-Algebraic Structure of Quantum Stochastic Integrals in Fock Space. Rediconti del Sem. Mat. e Fis. di Milano, LVIII, 177-193 (1988).

[80] G. Ludwig, Math. Phys., 4, 331 (1967), 9, 1 (1968).

[81] E. B. Davies \& J. Lewis, Comm. Math. Phys., 17, 239-260 (1970).

[82] E. B. Ozawa, J. Math. Phys., 25, 79-87 (1984).

[83] V. P. Belavkin, Quantum Stochastic Calculus and Quantum Nonlinear Filtering. Journal of Multivariate Analysis, 42, No. 2, 171-201 (1992).

[84] V. P. Belavkin \& P. Staszewski. Rep. Math. Phys., 29, 213 (1991).

[85] V. P. Belavkin \& O. Melsheimer, A Stochastic Hamiltonian Approach for Quantum Jumps, Spontaneous Localizations, and Continuous Trajectories. Quantum and Semiclassical Optics 8, 167-187 (1996).

[86] V. P. Belavkin, Quantum Continual Measurements and a Posteriori Collapse on CCR. Com. Math. Phys., 146, 611-635 (1992).

[87] L. Accardi, A. Frigerio \& J. Lewis, Publ. RIMS Kyoto Univ., 18, 97 (1982).

[88] R. L. Stratonovich, Conditional Markov Processes and Their Applications to Optimal Control. Moscow State University, Moscow 1966.

[89] V. P. Belavkin, A New Wave Equation for a Continuous Nondemolition Measurement. Phys. Lett. A, 140, 355-358 (1989).

[90] V. P. Belavkin, A Stochastic Posterior Schrödinger Equation for Counting Nondemolition Measurement. Letters in Math. Phys. 20, 85-89 (1990). 
[91] A. Barchielli \& V. P. Belavkin, Measurements Continuous in Time and a posteriori States in Quantum Mechanics. J. Phys. A: Math. Gen. 24, 1495-1514 (1991).

[92] I. Persival, Quantum State Diffusion. Cambridge University Press, 1999.

[93] V. P. Belavkin \& P. Staszewski, A Quantum Particle Undergoing Continuous Observation. Phys. Let. 140, 359-362 (1989).

[94] V. P. Belavkin \& P. Staszewski, Nondemolition Observation of a Free Quantum Particle. Phys. Rev. 45, No. 3, 1347-1356 (1992).

[95] D. Chruscinski \& P. Staszewski, On the Asymptotic Solutions of the Belavkin's Stochastic Wave Equation. Physica Scripta. 45, 193-199 (1992).

[96] V. N. Kolokoltsov, Scattering Theory for the Belavkin Equation Describing a Quantum Particle with Continuously Observed Coordinate. J. Math. Phys. 36 (6), 2741-2760 (1995).

[97] V. P. Belavkin, A Dynamical Theory of Quantum Continuous Measurements and Spontaneous Localizations. Russ Journ of Math Phys 3 (1), 3-24 (1995).

[98] V. P. Belavkin \& P. Staszewski, Quantum Stochastic Differential Equation for Unstable Systems. J. Math. Phys. 41 (11), 7220-7233 (2000).

[99] V. P. Belavkin, In: New Development of Infinite-Dimensional Analysis and Quantum Probability, RIMS Kokyuroku 1139, 54-73, April, 2000; Quantum Stochastics, Dirac Boundary Value Problem, and the Inductive Stochastic Limit. Rep. Math. Phys., 46, No.3, 2000.

[100] V. P. Belavkin, In: Evolution Equations and Their Applications in Physical and Life Sciences, G Lumer and L. Weis eds., Lect. Notes in Pure and Appl. Math. 215, 311-327 (Marcel Dekker, Inc., 2001).

[101] V. P. Belavkin \& V. N. Kolokoltsov, Stochastic Evolutions as Boundary Value Problems. In: Infinite Dimensional Analysis and Quantum Probability, Research Institute for Mathematical Studies, Kokyuroku 1227, 83-95 (2001).

[102] E. Schrödinger, Sitzberg Press Akad. Wiss. Phys.-Math. Kl. 144-53 (1931).

[103] J. G. Cramer, Rev. Mod. Phys., 58, 647-87 (1986).

[104] J. Dixmier, Von Neumann Algebras.North Holand, Amsterdam, (1981)

[105] V. P. Belavkin, P. Staszewski, Relative Entropy in $C^{*}$-algebraic Statistical Mechanics. Reports in Mathematical Physics, 20, 373-384 (1984).

[106] L. Accardi, Noncommutative Markov Chains, Proc. of Int. School of Mathematical Physics, Camerino, 266-295 (1974).

[107] B. Kümmerer, On the Structure of Markov Dilations on $W^{*}$-algebras. In: Quantum Probability and Applications II, eds. L. Accardi and W. von Waldenfels, 228-244 (Springer, 1988).

[108] B. Kümmerer \& H. Maassen, The Essentially Commutative Dilations of Dynamical Semigroups on $M_{n}$, Commun. Math. Phys. 109, 1-22 (1987).

[109] V. P. Belavkin, P. Staszewski, A Radon-Nikodym Theorem for Completely Positive Maps. Reports in Mathematical Physics, 24, 49-55 (1986).

[110] K. Itô, On a Formula Concerning Stochastic Differentials. Nagoya Math . J., 3, 55-65, (1951).

[111] V. P. Belavkin, On Quantum Itô Algebras and Their Decompositions. Lett. in Math. Phys. 45, 131-145 (1998).

[112] V. P. Belavkin, A Quantum Nonadapted Itô Formula and Stochastic Analysis in Fock Scale. J. Func. Anal., 102, No. 2, 414-447 (1991).

[113] V. P. Belavkin, The Unified Itô Formula Has the Pseudo-Poisson Structure. J. Math. Phys. 34, No. 4, 1508-18 (1993).

[114] C. W. Gardiner, A. S. Parkins, \& P. Zoller. Phys. Rev. A 46, 4363 (1992).

[115] R. Dunn, A. S. Parkins, P. Zoller, \& C. W. Gardiner. Phys. Rev. A 46, 4382 (1992).

[116] V. P. Belavkin, On Stochastic Generators of Completely Positive Cocycles. Rus. J. Math. Phys., 3, 523-528 (1995).

[117] V. P. Belavkin, Quantum Stochastic Positive Evolutions: Characterization, Construction, Dilation. Comm. Math. Phys., 184, 533-566 (1997).

[118] G. Lindblad, On The Generators of Quantum Stochastic Semigroups. Commun. Math. Phys., 48, pp. 119-130 (1976).

[119] A. Pais, Niels Bohr's Times, Clarendon Press - Oxford 1991.

[120] D. C. Cassidy, Uncertainty. Werner Heisenberg. W. H. Freeman, New-York, 1992.

[121] M. Jammer, The Conceptual Development of Quantum Mechanics. McGraw-Hill, 1966. 
Mathematics Department, University of Nottingham, NG7 2RD, UK

E-mail address: vpb@maths.nott.ac.uk

$U R L:$ http://www.maths.nott.ac.uk/personal/vpb/ 